\title{
A holistic approach to carbon-enhanced metal-poor stars ${ }^{\star}$
}

\author{
T. Masseron ${ }^{1,2}$, J. A. Johnson ${ }^{1}$, B. Plez ${ }^{2,3}$, S. Van $\mathrm{Eck}^{4}$, F. Primas ${ }^{5}$, S. Goriely ${ }^{4}$, and A. Jorissen ${ }^{4}$ \\ 1 The Ohio State University, Columbus, OH, USA \\ e-mail: masseron@astronomy .ohio-state.edu \\ 2 GRAAL, Université Montpellier II, 34095 Montpellier Cedex 05, France \\ 3 Department of Physics and Astronomy, Uppsala Astronomical Observatory, Box 515, 75120 Uppsala, Sweden \\ 4 Institut d'Astronomie et d'Astrophysique, Université Libre de Bruxelles, Belgium \\ 5 European Southern Observatory (ESO), Karl-Schwarzschild-Str. 2, 85749 Garching b. München, Germany
}

Received 29 January 2009 / Accepted 3 July 2009

ABSTRACT

\begin{abstract}
Context. Carbon-enhanced metal-poor (CEMP) stars are known to have properties that reflect the nucleosynthesis of the first lowand intermediate-mass stars, because most have been polluted by a now-extinct AGB star.

Aims. By considering abundances in the various CEMP subclasses separately, we try to derive parameters (such as metallicity, mass, temperature, and neutron source) characterising AGB nucleosynthesis from the specific signatures imprinted on the abundances, and separate them from the impact of thermohaline mixing, first dredge-up, and dilution associated with the mass transfer from the companion.

Methods. To place CEMP stars in a broader context, we collect abundances for about 180 stars of various metallicities (from solar to $[\mathrm{Fe} / \mathrm{H}]=-4$ ), luminosity classes (dwarfs and giants), and abundance patterns (e.g. C-rich and poor, Ba-rich and poor), from both our own sample and the literature.

Results. We first show that there are CEMP stars that share the properties of CEMP-s stars and CEMP-no stars (which we refer to as CEMP-low-s stars). We also show that there is a strong correlation between $\mathrm{Ba}$ and $\mathrm{C}$ abundances in the s-only CEMP stars. This represents a strong detection of the operation of the ${ }^{13} \mathrm{C}$ neutron source in low-mass AGB stars. For the CEMP-rs stars (seemingly enriched with elements from both the $s$ - and $r$-processes), the correlation of the $\mathrm{N}$ abundances with abundances of heavy elements from the 2nd and 3rd s-process peaks bears instead the signature of the ${ }^{22} \mathrm{Ne}$ neutron source. Since CEMP-rs stars also exhibit O and $\mathrm{Mg}$ enhancements, we conclude that extremely hot conditions prevailed during the thermal pulses of the contaminating AGB stars. We also note that abundances are not affected by the evolution of the CEMP-rs star itself (especially by the first dredge-up). This implies that mixing must have occurred while the star was on the main sequence, and that a large amount of matter must have been accreted so as to trigger thermohaline mixing. Finally, we argue that most CEMP-no stars (with neutron-capture element abundances comparable to non-CEMP stars) are likely the extremely metal-poor counterparts of CEMP neutron-capture-rich stars. We also show that the $\mathrm{C}$ enhancement in CEMP-no stars declines with metallicity at extremely low metallicity $([\mathrm{Fe} / \mathrm{H}]<-3.2)$. This trend is not predicted by any of the current AGB models.
\end{abstract}

Key words. stars: abundances - stars: AGB and post-AGB - stars: population II

\section{Introduction}

The largest wide-field spectroscopic surveys for metal-poor stars to date, the HK survey (Beers et al. 1992) and the HES survey (Christlieb et al. 2001), have provided a tremendous wealth of information for the study of the early chemical evolution of our Galaxy. One of the most interesting and surprising result is the high frequency of carbon-enhanced stars $([\mathrm{C} / \mathrm{Fe}]>1.0$, hereafter CEMP stars) among metal-poor stars. The results of the HK and HES surveys indicate that they represent 20-30\% of stars with $[\mathrm{Fe} / \mathrm{H}]<-2.5$ (Lucatello et al. 2005b, 2006). This finding has prompted a number of high-resolution, high signal-to-noise studies aimed at understanding the origin of the abundance anomalies in these objects. The carbon-enhancement phenomenon appears in stars that exhibit four different heavyelement abundance patterns:

(i) The most numerous class is characterised by enrichments of neutron-capture elements. From radial-velocity variations,

* Tables $1-4$ are only available in electronic form at http://www . aanda. org
Preston \& Sneden (2000) and Lucatello et al. (2005b) demonstrate that these stars are members of binary systems. Some of them (hereafter CEMP-s) also exhibit an abundance pattern of neutron-capture elements compatible with the operation of the $s$-process in asymptotic giant branch (AGB) stars. Thus, it is now established that these CEMPs stars - as well as classical CH stars - are members of wide binary systems, where the former primary star transferred material during its AGB phase onto the presently observable companion (McClure \& Woodsworth 1990). We indeed demonstrate in Masseron et al. (in prep., hereafter Paper II) that CEMP and $\mathrm{CH}$ stars belong to the same class of stars.

(ii) Another class of CEMP stars (hereafter CEMP-rs), exhibiting large overabundances of elements produced by the $s$ process and of elements traditionally related to the $r$-process, was discovered by Barbuy et al. (1997) and Hill et al. (2000). A number of these stars exhibit radial-velocity variations (e.g., Paper II; Sivarani et al. 2004; Barbuy et al. 2005). There is no doubt that these stars are binaries (they might even be triple systems) and that the companion(s?) is(/are) 
responsible for the peculiar abundance pattern. Nevertheless, CEMP-rs stars are very puzzling: they have a large Ba enhancement, representative of the $s$-process, and a very large Eu enhancement, which is representative of the $r$-process. Most of the scenarios invoked to explain the peculiar rs abundance pattern include a double phase: a $r$-process pollution (from a type-II supernovae) followed by a $s$-process pollution (from an AGB star) or vice versa (see hypotheses III, IV, and VI of Jonsell et al. 2006, and references therein). These scenarios are supported by the predictions of Bisterzo et al. (2006) which satisfactorily reproduce the general features of CEMP-rs neutron-capture patterns by assuming in their model an initial high $r$-process enrichment before slow neutron capture begins. In contrast, Johnson \& Bolte (2004) and Masseron (2006) do not find a satisfactory combination of $r$ - and $s$-process that reproduce the neutron-capture element pattern in CEMP-rs stars, and call for a modified neutroncapture process. In addition, the large number of CEMP-rs stars observed at low metallicities casts doubt on the likelihood of two-phase scenarios. From the present analysis, we suggest instead that the CEMP-rs stars are produced by intermediate-mass AGB stars, where both ${ }^{13} \mathrm{C}(\alpha, \mathrm{n}){ }^{16} \mathrm{O}$ and ${ }^{22} \mathrm{Ne}(\alpha, \mathrm{n}){ }^{16} \mathrm{O}$ neutron sources operate.

(iii) Some CEMP stars with no enhancements in their neutroncapture-element abundances have been identified (hereafter CEMP-no; Aoki et al. 2002c). Unfortunately, not many of these stars currently have enough radial-velocity measurements to constrain their binary properties. Consequently, a mass-transfer scenario comparable to that operating in CEMP-s (and possibly in CEMP-rs) stars is not firmly established. Nevertheless, the origin of this category is of great interest as the two most Fe-poor stars known to date (HE 01075240 and HE 1327-2326; Christlieb et al. 2002; Frebel et al. $2005)$ belong to this class. The existence of a very large $C$ content in extremely low-metallicity stars may be explained from nucleosynthesis processes operating in basically all mass ranges, including notably hypernovae (e.g., Heger \& Woosley 2002), type-II supernova models (e.g., Woosley \& Weaver 1995; Limongi \& Chieffi 2003), massive stars with rotation accounting for the $\mathrm{N}$ enhancement (e.g., Meynet \& Maeder 2002; Hirschi 2007), fast-rotating AGB models (e.g., Siess et al. 2004), early-AGB or Red-Giant-Branch (RGB) stars with modified helium-core flashes at very low metallicity (e.g., Fujimoto et al. 2000; Suda et al. 2004). The intriguing possibility that the abundance pattern of the two most metal-poor stars known to date is actually determined by chemical segregation rather than nucleosynthesis was raised by Venn \& Lambert (2008).

(iv) Finally, a single case of a highly $r$-process-enhanced CEMP star has been noted (CS22892-052, Sneden et al. 2003b).

CEMP stars provide an extremely interesting insight into the initial mass function (IMF) of the early Galaxy. Those CEMP stars, which have been polluted by AGB stars probe the IMF of the intermediate-mass $\left(\sim 1-8 M_{\odot}\right)$ stars, provided of course that a reliable mass may be assigned to the companion AGB star. Theoretical models have indeed shown several ways that the mass of the AGB star may influence nucleosynthesis. For example, $\mathrm{N}$ is strongly enhanced by Hot-Bottom Burning (HBB) at the expense of $\mathrm{C}$ in intermediate-mass $\left(\sim 3-8 M_{\odot}\right)$ AGB stars. On the other hand, $\mathrm{F}$ is produced only in low-mass AGB stars (see other examples in Karakas \& Lattanzio 2007). For the production of the heavy elements by means of the $s$-process, the mass of the AGB star is also of importance. It has been suggested that low-mass $\left(\sim 1-3 M_{\odot}\right)$ AGB stars produce neutrons mainly by the ${ }^{13} \mathrm{C}(\alpha, n){ }^{16} \mathrm{O}$ reaction operating in radiative conditions (Straniero et al. 1995), whereas in intermediate-mass AGB stars, a weak $s$-process is driven by ${ }^{22} \mathrm{Ne}(\alpha, \mathrm{n})^{25} \mathrm{Mg}$ operating in convective conditions (Goriely \& Siess 2004, 2005). However, no AGB model predicting the formation of the necessary ${ }^{13} \mathrm{C}$ pocket in a self-consistent way is yet available, and the ${ }^{22} \mathrm{Ne}(\alpha, \mathrm{n})^{25} \mathrm{Mg}$ reaction rate is still affected by large uncertainties.

Although yields are not yet available for a wide range of elements, stellar masses, and metallicities, some attempts have been made to compare AGB yields for different masses with CEMP star abundances. Bisterzo \& Gallino (2008) derived the mass of the former AGB companion of 74 CEMP-s stars by fitting abundance patterns with their predictions. They found that all abundance anomalies in CEMP-s stars originate in AGB stars with $M<1.4 M_{\odot}$. Based on Fujimoto et al. (2000) calculations, Komiya et al. (2007) argued that CEMP-s stars had an AGB companion with $0.8 M_{\odot}<M<3.5 M_{\odot}$, whereas CEMP-no stars have an intermediate-mass AGB companion with $M>3.5 M_{\odot}$. However, none of these models are able to reproduce the properties of the 4 CEMP subclasses simultaneously.

In this paper, we review the CEMP phenomenon to shed light on the origin of these intriguing stars based on an unprecedented compilation of abundances of all CEMP classes. After classifying the sample in different categories according to their observed abundances, we first provide additional proof that Ba stars are formed by mass-transfer from metal-rich AGB stars (Sect. 3). Thus, Ba stars represent a metal-rich sample that can be compared with CEMP stars. We also discuss the need for introducing CEMP-low-s stars, which show low $s$-process element abundances (Sect. 4). Our approach consists of looking at element correlations between element abundances in each CEMP class. In this manner, we provide new insights into the nucleosynthesis in the AGB companion of CEMP-s stars (Sect. 5). Assuming that AGB stars are also responsible for the peculiar composition of CEMP-rs stars, we try to identify their nucleosynthesis processes (Sect. 6). In parallel, we qualitatively compare the mean trends between CEMP classes and with non Crich stars. Finally, we discuss the nature of the companion of CEMP-no stars and evaluate the role of AGB stars at extremely low-metallicity $([\mathrm{Fe} / \mathrm{H}]<-3.0)$ (Sect. 7). This holistic view of CEMP abundances finally attempts to draw a coherent picture of AGB nucleosynthesis at low metallicity (Sect. 8).

\section{The extended sample}

We compiled abundances from analyses of high-resolution spectra $(R>40000)$ of CEMP stars (Tables 1 and 2), Ba stars, and non-carbon-enhanced metal-poor stars (Tables 3 and 4). C-rich stars are defined as stars with $[\mathrm{C} / \mathrm{Fe}]>0.9^{1}$. In this paper, all plots are compiled exclusively of data from these tables, including our own data from Paper II. All these data are renormalised by the Asplund (2005) solar abundances.

Figure 1 shows the distribution of the stars from Tables 2 and 4 in the $([\mathrm{Ba} / \mathrm{Fe}],[\mathrm{Eu} / \mathrm{Fe}])$ diagram, which involves two neutron-capture elements. This figure clearly indicates reveals different families that can be distinguished (see Fig. 2 for details). Our definitions closely match those of Jonsell et al. (2006)

\footnotetext{
${ }^{1}$ Since not all authors have adopted the same solar abundances for $\mathrm{C}$ and $\mathrm{Fe}$, our CEMP criterion $([\mathrm{C} / \mathrm{Fe}]>0.9)$ slightly differs from the one used by Rossi et al. $(1999)([\mathrm{C} / \mathrm{Fe}]=1.0)$, to keep stars like the unique $r$-process-rich star CS 22892-052 in the CEMP family with the Asplund (2005) solar abundances adopted in the present paper.
} 
T. Masseron et al.: A holistic approach to carbon-enhanced metal-poor stars
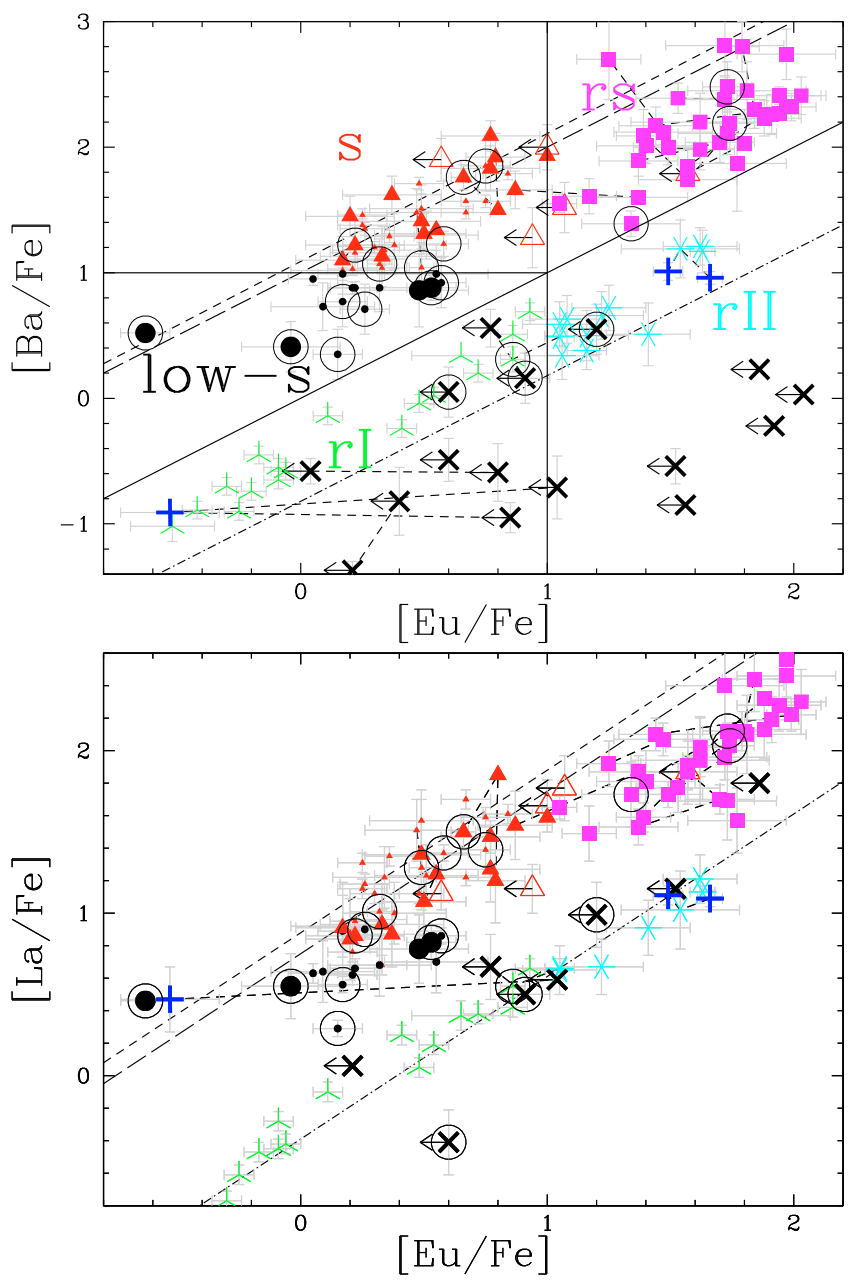

Fig. 1. $[\mathrm{Ba} / \mathrm{Fe}]$ vs. $[\mathrm{Eu} / \mathrm{Fe}]$ and $[\mathrm{La} / \mathrm{Fe}]$ vs. $[\mathrm{Eu} / \mathrm{Fe}]$ abundances in sample stars. The (red) triangles represent CEMP-s stars, the filled (black) circles are the CEMP-low-s stars, the (black) crosses are the CEMP-no stars and the (magenta) squares represent the CEMP-rs stars. We also represent classical $\mathrm{Ba}$ stars with tiny black dots and tiny red triangles. Large open circles identify stars studied in Paper II. The cyan solid lines separate the different classes (see Fig. 2 for their explicit definition). The black lines correspond to pure $s$-process nucleosynthesis predictions for a $0.8 M_{\odot}$ (short dash; Masseron et al. 2006) and a $3 M_{\odot}$ (long dash; Goriely \& Siess 2005) metal-poor AGB star and to pure solar $r$ process (dash-dot; Goriely 1999). Although La is an excellent $s$-process tracer, there are fewer abundances available for this element in the literature. Note that the star HE 2356-041 has a typical s-process $\mathrm{La} / \mathrm{Eu}$ ratio (lower panel), despite $\mathrm{Ba} / \mathrm{Eu}$ ratio is inferring it as a CEMP-r star (upper panel; see also Sect. 4).

for CEMP-s and CEMP-rs stars, and those of Beers \& Christlieb (2005) for CEMP-no, rI, and rII stars (see below for a definition of rI and rII stars). However, these studies do not consider stars with either no Eu abundance available or with only an upper limit. Thus, to classify those, we rely on the Ba abundance alone. In Fig. 1, we labelled four stars as CEMP-low-s stars, three of them being based on an Eu measurement from Paper II (see Sects. 4 and 7). At this stage, based on the consideration of Fig. 1 only, the necessity of distinguishing CEMP-low-s stars from CEMP-no stars is not at all obvious, since it may appear as simply resulting from the absence of a firm Eu detection in CEMP-no stars. This question will be addressed in more detail in Sect. 7.2.

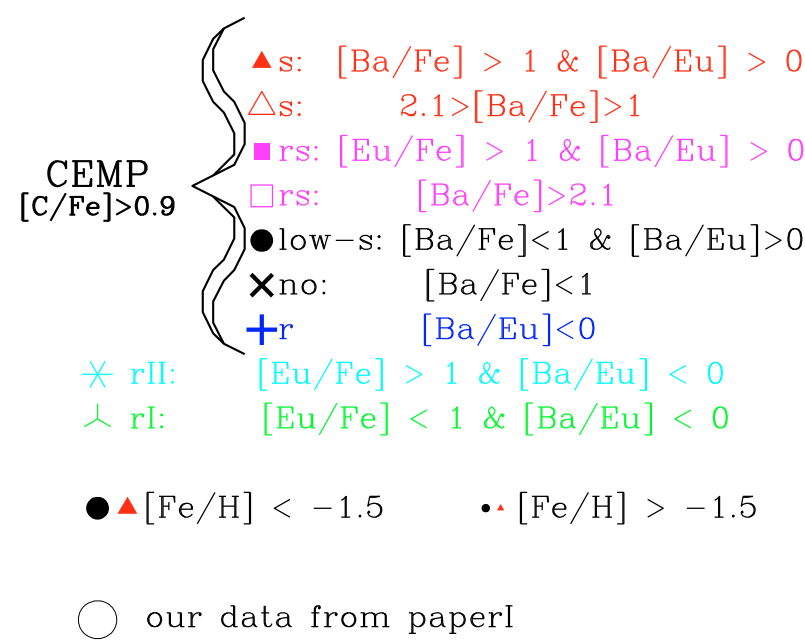

Fig. 2. The adopted classification results from Fig. 1. The classification and the corresponding colour-shape code adopted in this figure are used in all the figures of this paper. Large symbols and small symbols correspond to $[\mathrm{Fe} / \mathrm{H}]\langle-1.5$ and $\rangle-1.5$, respectively. Open symbols are used whenever there is no Eu abundance available. In this case, the classification is solely based on the Ba abundance displayed in Fig. 1.

The stars denoted rI and rII by Beers \& Christlieb (2005) represent the majority of metal-poor stars (CEMP stars being 20\% of them). Because they apparently do not belong to binary systems, we assume that their abundances are representative of the composition of the interstellar medium from which these metalpoor stars formed, and hence of the initial composition of CEMP stars.

In the whole sample, we identify 47 CEMP-s, 44 CEMPrs, and 42 CEMP-no stars, including 32 multiple measurements, summing up to a total of 101 different CEMP stars. Despite all stars come from only 2 surveys of metal-poor stars (HK and HES), we do not attempt to extract accurate frequencies from these numbers because each author has potentially biased the subsample of stars with an abundance analysis by using specific selection criteria. There are also 2 CEMP stars, which show a pure $r$-process Ba/Eu ratio (CS 22892-052 and HE 2356-0410, represented by blue "plus" symbols). It is possible that these stars have received some C-rich material from an AGB star, but the high $r$-process initial composition overwhelms the low $s$-process elemental abundances, as suggested by Aoki et al. (2002c). We also note that the latter star (aka CS 22957-027) has a $\mathrm{La} / \mathrm{Eu}$ ratio compatible with a pure $s$-process (lower panel of Fig. 1), making it difficult to identify the origin of the neutroncapture elements. We also emphasise that, among three high resolution spectroscopic analyses of this star, only one study was able to derive the Eu abundance at a very low level, with Eu lines falling in a forest of strong $\mathrm{CN}$ lines. Therefore, the accuracy of this measurement should perhaps be carefully be re-examined.

The error bars shown in all the plots are generally the random errors published by the authors (when available) and are very often in the range $\approx 0.1-0.2$ dex. We do not include systematic errors, because we consider that they are most accurately represented by the dispersion in the abundances resulting from the different studies of a given object (connected by long-dashed lines in all the figures). In Paper II, we show that systematic errors caused by different assumptions about model-atmosphere parameters may produce large abundance discrepancies, especially for abundance determinations based on a single line. As an example, the $[\mathrm{Pb} / \mathrm{Fe}]$ ratio derived in different studies of 

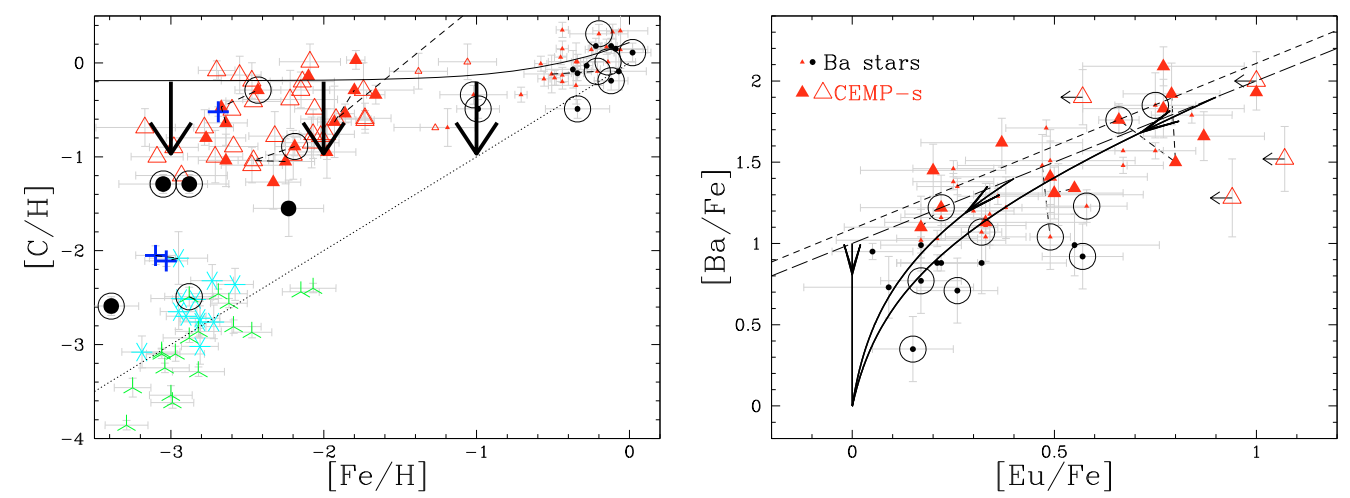

Fig. 3. (top panel) The $\mathrm{C}$ content in CEMP-s stars and in Ba stars (see Fig. 2 for a description of symbols). The dotted line represents the Galactic average $\mathrm{C}$ content $([\mathrm{C} / \mathrm{Fe}] \approx 0)$ and the solid line stands for a constant amount of $\mathrm{C}([\mathrm{C} / \mathrm{H}]=-0.2)$ added to the initial Galactic average content. The black arrows represent a arbitrary dilution factor of the accreted $\mathrm{C}$. This assumption of a constant $\mathrm{C}$ abundance in the accreted material is consistent with a primary $\mathrm{C}$ production in AGB stars. This simple calculation shows that Ba stars are indeed the analogues of $\mathrm{C}$ stars at high metallicities. (bottom panel) $[\mathrm{Ba} / \mathrm{Fe}]$ as a function of $[\mathrm{Eu} / \mathrm{Fe}]$ for Ba stars and CEMP-s/low-s stars. The black arrows represent the track followed by the abundance ratios when increasing the dilution of $s$-process enriched material in a solar-composition material. This demonstrates that a varying dilution of $s$-process-rich material into solar-composition material can explain the global trend of $[\mathrm{Ba} / \mathrm{Fe}]$ and $[\mathrm{Eu} / \mathrm{Fe}] \mathrm{ratios}$ in $\mathrm{Ba}$ stars.

HE 2158-0348 ranges from 2.77 to 3.42 , while the $[\mathrm{Ba} / \mathrm{Fe}]$ values span the much narrower range 1.60-1.66.

Because of the various oxygen-abundance diagnostics, we choose to plot $\mathrm{O}$ abundances uncorrected for NLTE and 3D effects. However, we give in Col. 11 of Table 1 NLTE corrections for O I measurements using the formula of Takeda (2003). The corrections for $\mathrm{O}$ triplet measurements are of the order of 0.2 dex, in agreement with García Pérez et al. (2006). We note that for some of the stars, especially the CEMP-rs, the O-line equivalent widths are above the applicability limit of the formula. García Pérez et al. (2006) also found 3D corrections for $\mathrm{OH}$ of the order of -0.1 dex.

Although the production of elements such as $\mathrm{C}, \mathrm{N}$ and $s$ process elements in the AGB star cause the abundance to become significantly higher than that already present in the CEMP star, abundance ratios such as $[\mathrm{X} / \mathrm{Fe}]$ or $[\mathrm{X} / \mathrm{H}]$ in CEMP stars are not necessarily identical to the initial yields of the AGB star, especially when not much matter is accreted and/or is heavily diluted in the CEMP star envelope. Various mass-transfer efficiencies are indeed expected. Because of the large variety of orbital parameters, various accretion and dilution factors contribute to the abundance scatter observed for $\mathrm{C}, \mathrm{N}$, or $s$-process elements. Unfortunately, information for only very few orbits are available for CEMP stars. Therefore, it is difficult to constrain the initial abundances from observations of $[\mathrm{X} / \mathrm{Fe}]$ or $[\mathrm{X} / \mathrm{H}]$ ratios. Nevertheless, the reader should recall that using abundance ratios of enhanced $s$-process elements (e.g., [Ba/Eu], [Pb/Ba]) represents a way of partially reducing the uncertainty produced by the various mass transfer efficiencies and to understanding the AGB nucleosynthesis.

\section{Ba stars and CEMP-s stars}

Jorissen \& Van Eck (2000) demonstrate that Ba stars are just part of a binary evolutionary sequence, which also involves MS, $\mathrm{S}$, and $\mathrm{C}$ stars, without lines from the unstable element Tc (see their Fig. 1), and Allen \& Barbuy (2006b) conclude that Ba stars have the same $s$-process signature as AGB stars. Figure 1 shows that they exhibit $[\mathrm{Ba} / \mathrm{Fe}]$ and $[\mathrm{Eu} / \mathrm{Fe}]$ ratios identical to those of CEMP-s stars. Following Cohen et al. (2006), we thus suggest that CEMP-s stars and Ba stars belong to the same category of AGB mass-transfer stars, and differ only on the basis of their metallicity. The Ba stars are not as carbon-enhanced as CEMP-s stars because their composition prior to mass transfer from the AGB star, already consisted of a high $\mathrm{C}$ and $\mathrm{O}$ content, and, in these circumstances, the $\mathrm{C}$ present in the accreted material is insufficient to bring the resulting $\mathrm{C} / \mathrm{O}$ ratio above unity. Hence, even after the transfer of $\mathrm{C}$-rich material from the AGB companion, the $[\mathrm{C} / \mathrm{H}]$ ratio remains close to the Galactic average (dotted line in Fig. 3). Thus, the $\mathrm{C} / \mathrm{O}$ ratio remains below 1 , and $\mathrm{CH}$ or $\mathrm{C}_{2}$ lines are less intense in the spectra of $\mathrm{Ba}$ stars than in their more metal-poor counterparts. Therefore, we argue that the same nucleosynthesis processes are responsible for the $\mathrm{C}$ and selement production in Ba stars and CEMP-s stars, which just differ in terms of metallicity. We note that in the following plots we include Ba stars to give a broader view of AGB nucleosynthesis, thus making it possible to identify the impact of metallicity. In Fig. 3, we also highlight the effect of dilution (either in the AGB envelope or when the material transferred from the AGB star is mixed with material in the companion's envelope). We calculate the dilution tracks of the neutron-capture elements as follows:

$$
\begin{aligned}
& E u=(1-d) \times E u_{\mathrm{s}}+d \times E u_{\text {init }} \\
& B a=\left.\frac{B a}{E u}\right|_{\mathrm{s}} \times(1-d) \times E u_{\mathrm{s}}+\left.\frac{B a}{E u}\right|_{\text {init }} \times d \times E u_{\text {init }},
\end{aligned}
$$

where $d$ is the dilution factor (ranging from 0 to 1) and $E u$ and $B a$ are the resulting $\mathrm{Ba}$ and $\mathrm{Eu}$ abundances after dilution. We chose $\left.\frac{B a}{E u}\right|_{\mathrm{s}}$, so that $[\mathrm{Ba} / \mathrm{Eu}]_{\mathrm{s}}=1$, as observed in CEMP-s stars, and $\left.\frac{B a}{E u}\right|_{\text {init }}$ and $E u_{\text {init }}$, so that $[\mathrm{Ba} / \mathrm{Eu}]_{\text {init }}=0$ and $[\mathrm{Eu} / \mathrm{Fe}]=0$ as expected for solar metallicity. We apply this formula to 3 values of the $s$-process element $\mathrm{Eu}\left(E u_{\mathrm{s}}\right)$ so that $[\mathrm{Eu} / \mathrm{Fe}]_{\mathrm{s}}=0.0,0.4,0.9$, matching the observed range. This simple calculation demonstrates that the scatter observed for the neutron-capture elements in Ba stars and for C in CEMP-s stars may at least be partly ascribed to dilution.

\section{CEMP-Iow-s stars: the low s-process counterparts of CEMP-s stars}

The discovery of CEMP stars that have low $\mathrm{Ba}$ abundances (black crosses in Fig. 3) was exciting, because, as discussed in Sect. 1, it suggested that the carbon enrichment seen in these stars could be due to pollution by a star other than an AGB. 


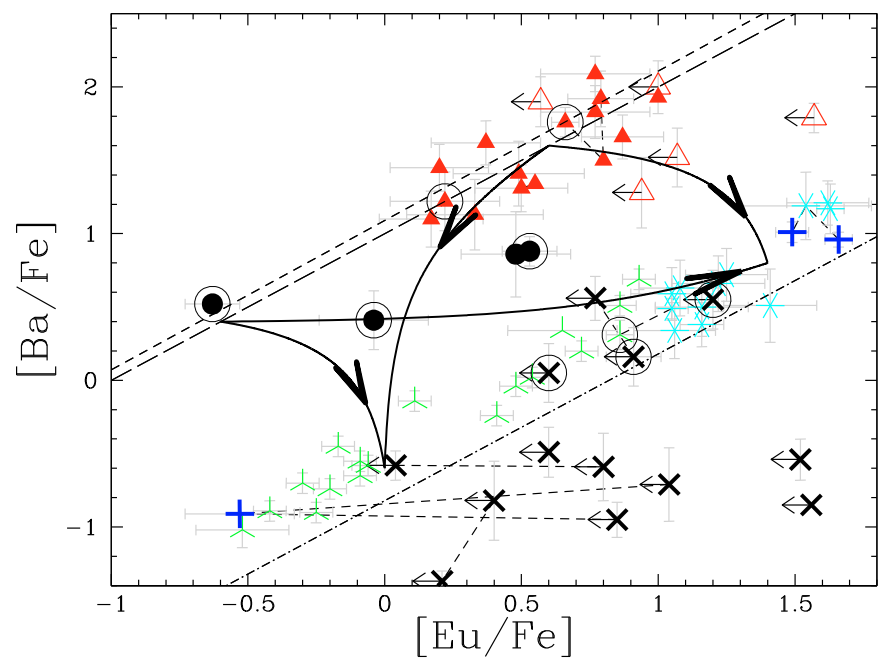

Fig. 4. $\mathrm{Ba} / \mathrm{Fe}$ and $\mathrm{Eu} / \mathrm{Fe}$ in metal-poor stars (see Fig. 2 for a description of symbols). The black arrows represent the track followed by the abundance ratios when increasing the dilution of $s$-process enriched material in a pure $r$-process material. Because the initial pure $s$-process composition and the pure $r$-process composition are unknown, this calculation as been made for 2 sets of $s$-process $\mathrm{Ba}$ and $\mathrm{Eu}$ abundances that are representative of that observed in CEMP-s and CEMP-low-s stars, and 2 sets of $r$-process $\mathrm{Ba}$ and Eu abundances typical of that observed in rI and rII stars.

The identification of the origin of these stars with low Ba abundances represents a special challenge, because very few abundance data are available for the neutron-capture elements. This difficulty may be caused either by a true absence of large overabundances, or to the difficulty in detecting spectral lines when the metallicity is very low, even in the presence of overabundances. This situation is illustrated by Fig. 1, where stars currently classified as CEMP-no stars (large crosses) only have an upper limit to their Eu abundances. In Paper II and in Masseron et al. (2006), we derived the Eu abundances for three CEMP-no stars (HE 1419-1324, HE 1001-0243, and CS 30322-023, represented by large circled black dots). Their low Ba abundances would classify them as CEMP-no stars according to Beers \& Christlieb (2005).

There is yet another star, namely HKII 17435-00532, with properties similar to the three just described (large circled black dots in Fig. 4). So, in addition to high C, they differ from "normal" field stars in terms of their their heavy-element abundance. These stars fall between the $s$ - and $r$-process lines, thus may be accounted for by diluting pure $s$-process material in pure $r$ process matter. We therefore identified stars with low $\mathrm{Ba}$ enhancements similar to those in CEMP-no stars, but with $[\mathrm{Ba} / \mathrm{Eu}]$ ratios showing evidence of contamination by $s$-process material. We labelled these as CEMP-low-s stars.

We emphasise that, because the dilution lines (arrows in Fig. 4) cross the region between the $s$-process and $r$-process lines, CEMP-low-s stars could erroneously be classified as either CEMP-rs or CEMP-no stars. So far, not many CEMP-low-s stars are known, but we suggest in Sect. 7.2 that a fair fraction of CEMP-no stars could actually be CEMP-low-s stars when Eu abundances become available.

The situation differs a little for CS 30322-023. Although its low $[\mathrm{Ba} / \mathrm{Fe}]$ ratio formally locates it among CEMP-low-s stars, it falls along the pure $s$-process line, so that there is no need to invoke the above argument involving dilution. CS 30322-023 is also special in being a genuine low-metallicity AGB star, with a very low initial Eu content (Paper II and Masseron et al. 2006)! Thus, there has been no dilution associated with mass-transfer from an AGB companion for this star, and its envelope contains pure $s$-processed matter brought there by the third dredge-up. All the Eu initially present has been overwhelmed by the $s$-process Eu brought by the dredged-up matter.

In the remainder of this paper, the four stars forming the CEMP-low-s category are included with the CEMP-s and CEMP-no stars in figures and discussions.

\section{The nature of the companion of CEMP-s stars}

The good agreement between the predicted and observed $[\mathrm{Ba} / \mathrm{Eu}]$ and $[\mathrm{La} / \mathrm{Eu}]$ ratios in CEMP-s and CEMP-low-s stars (Fig. 1) supports the standard model of the operation of the $s$-process in AGB stars by means of the ${ }^{13} \mathrm{C}(\alpha, \mathrm{n}){ }^{16} \mathrm{O}$ neutron source (Straniero et al. 1995; Goriely \& Mowlavi 2000). Consequently, we expect the $\mathrm{Ba} / \mathrm{C}$ ratio to depend on metallicity (Clayton 1988). This is because $\mathrm{C}$ is of primary origin (independent of metallicity; because of the triple- $\alpha$ reaction in the He-burning shell), whereas the production of $\mathrm{Ba}$ is secondary (it depends on the availability of Fe seed nuclei). The trend of $\mathrm{Ba}$ with metallicity predicted by the operation of the ${ }^{13} \mathrm{C}(\alpha, n){ }^{16} \mathrm{O}$ neutron source is, however, a complicated one. First, one should remark that the $\mathrm{Ba}$ abundance at the surface of AGB stars where the ${ }^{13} \mathrm{C}(\alpha, \mathrm{n}){ }^{16} \mathrm{O}$ process operates is affected by three different factors:

(i) The low number of available Fe seed nuclei is the major limiting factor at the lowest metallicities. Hence, the $[\mathrm{Ba} / \mathrm{C}]$ increases by up to $[\mathrm{Fe} / \mathrm{H}]=-1$ (Fig. 5 ).

(ii) The $s$-process abundance pattern (i.e., the ratio $[v h s / h s]$ where $v h s$ represents third-peak $s$-process elements such as $\mathrm{Pb}$ and $h s$ represents second-peak $s$-process elements such as $\mathrm{Ba}, \mathrm{La}$ or $\mathrm{Ce}$ ) also varies with metallicity, since the number of neutrons captured per seed nuclei increases with decreasing metallicity. This is because the ${ }^{13} \mathrm{C}(\alpha, \mathrm{n}){ }^{16} \mathrm{O}$ neutron source involves primary fuels, namely ${ }^{12} \mathrm{C}$ and protons, by ${ }^{12} \mathrm{C}(\mathrm{p}, \gamma){ }^{13} \mathrm{~N}\left(\beta^{+}\right){ }^{13} \mathrm{C}$. Hence, the number of available neutrons remains the same at all metallicities (see, however, item (iii) below). However, since the number of available Fe seed nuclei decreases with metallicity, at low metallicities the number of neutrons captured per Fe seed nuclei is large, and heavy $s$-process elements such as $\mathrm{Pb}$ are produced (see Fig. 6). At intermediate metallicities, however, the number of neutrons captured is just enough to synthesise second-peak elements such as $\mathrm{Ba}$. The $\mathrm{Ba}$ abundance should thus reach a maximum at intermediate metallicities.

(iii) The number of neutrons available will depend on the size of the proton pocket mixed in the carbon zone, which is currently not constrained by the models, since the physical mechanism responsible for the proton diffusion in the $\mathrm{C}$-rich shell remains unknown. The Ba enrichment predicted by the models is affected by this uncertainty, but the $\mathrm{C}$ enrichment is not. Hence, the $[\mathrm{Ba} / \mathrm{C}]$ ratio is not totally independent of mixing: although not affected by the third dredge-up, it is dependent on the size of the proton pocket, the variation of which may account for the scatter in s-process-element abundances such as Ce (left panel of Fig. 5).

Figure 5 shows that the $[\mathrm{Ba} / \mathrm{C}]$ ratio in CEMP-s stars qualitatively follows the expected trend, reaching its maximum value 

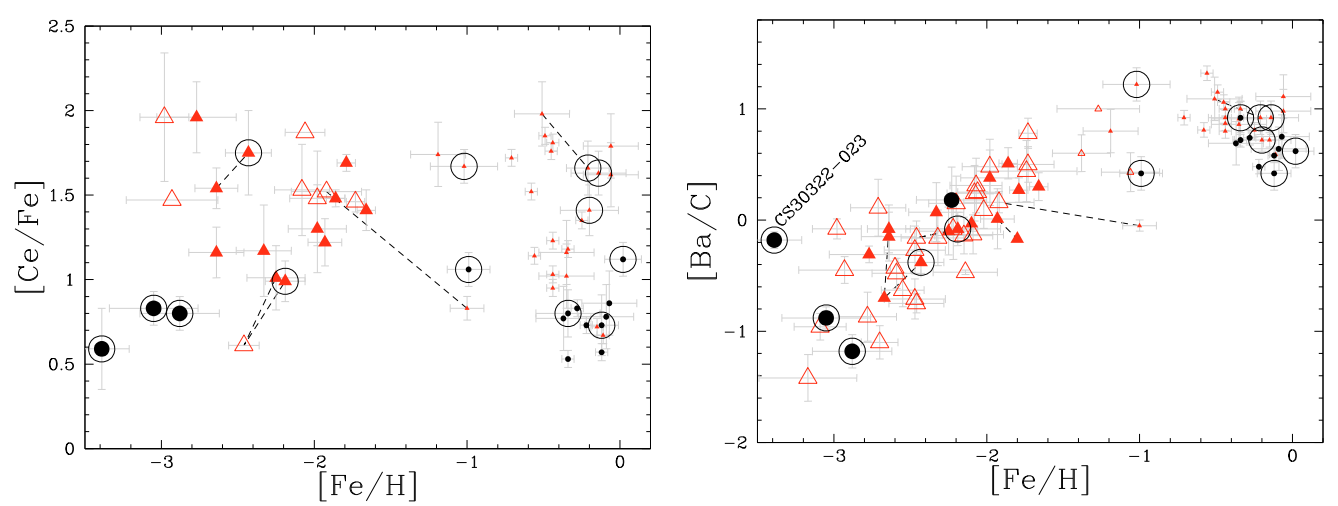

Fig. 5. $[\mathrm{Ce} / \mathrm{Fe}]$ (left panel) and $[\mathrm{Ba} / \mathrm{C}]$ (right panel) as a function of metallicity for CEMP-s and Ba stars (see Fig. 2 for a description of symbols). As a representative $s$-process element, Ce abundances exhibit significant scatter at all metallicities. Note that the right panel is equivalent to [Ba/Fe] vs $[\mathrm{C} / \mathrm{H}]$ (e.g., Fig. 8 of Aoki et al. 2002c) with the advantage of cancelling dilution and mass-transfer effects. There is a strong correlation between $\mathrm{Ba}$ and $\mathrm{C}$ synthesis. A maximum is obtained for $[\mathrm{Fe} / \mathrm{H}]$ between -1.0 and -0.6 as expected by Goriely \& Mowlavi (2000) and Busso et al. (2001).

around $[\mathrm{Fe} / \mathrm{H}]=-1^{2}$. The small scatter $(0.28 \mathrm{dex})$ in the $[\mathrm{Ba} / \mathrm{C}]$ versus $[\mathrm{Fe} / \mathrm{H}]$ relationship is indeed quite remarkable. It is comparable to the root-mean-square of the uncertainties in the measurements ( $\sim 0.2 \mathrm{dex})$. Therefore, the $\mathrm{Ba} / \mathrm{C}$ ratio produced by the $s$-process nucleosynthesis is not expected to vary much from star to star at a given metallicity. Although current AGB models explain the $[\mathrm{Ba} / \mathrm{C}]$ behaviour well as a function of metallicity, none of them reproduce its value quantitatively. Hence, our results place tight constraints on the proton-diffusion mechanism producing ${ }^{13} \mathrm{C}$ because this correlation links $\mathrm{C}$ production in the AGB with the $s$-process.

Concerning the $s$-process abundance pattern, improvements in the accuracy of the abundances have revealed that not all the elements in a given $s$-process peak behave similarly. Therefore, we choose to show single-element ratios in Fig. 6. This figure shows that the $\mathrm{Pb} / \mathrm{Ba}$ ratio is increasing as metallicity decreases, as expected from the models (Gallino et al. 1998; Goriely \& Mowlavi 2000; Busso et al. 2001). We emphasise in particular that three stars, formerly classified as CEMP-no for which we derived the $\mathrm{Pb}$ abundance (Paper II), fall along the expected trend; hence, they were reclassified as CEMP-low-s in Sect. 4.

However, these figures also exhibit a significant scatter about these broad trends. We remind the reader that $\mathrm{Pb}$ and $\mathrm{Ba}$ might be affected by observational uncertainties. Lead is very challenging to measure because it generally relies on one single line $(405.77 \mathrm{~nm})$ blended by a regular $\mathrm{CH}$ line $(405.78 \mathrm{~nm})$ and a broad CH-predissociation line (405.58 nm) (Plez et al. 2008), and is very sensitive to stellar parameters. The large spread in the $\mathrm{Pb}$ abundances derived by different authors within a given star, illustrates these difficulties. For example, CS 22942-019 was also analysed by Aoki et al. (2002c), and a large discrepancy was found between the upper limits to the $\mathrm{Pb}$ abundance derived by these two studies $(\approx 0.6$ dex). Ba might be affected by large measurement errors as illustrated by the different $[\mathrm{Ba} / \mathrm{Fe}]$ ratios in CS 22942-19, since most of the Ba lines used in spectroscopic studies are resonance lines, sensitive to non-LTE effects, and are usually quite strong lines.

There are also theoretical uncertainties. In the proton-mixing scenario (Goriely \& Mowlavi 2000), the [vhs/hs] ratio is essentially controlled by the metallicity. As shown by Van Eck et al. (2003), uncertainties originating from unknown dilution factors

\footnotetext{
2 As $[\mathrm{C} / \mathrm{N}] \approx 0$ in CEMP-s stars (see Sect. 6.4.1), identical values are found for $[\mathrm{Ba} / \mathrm{C}+\mathrm{N}]$.
}
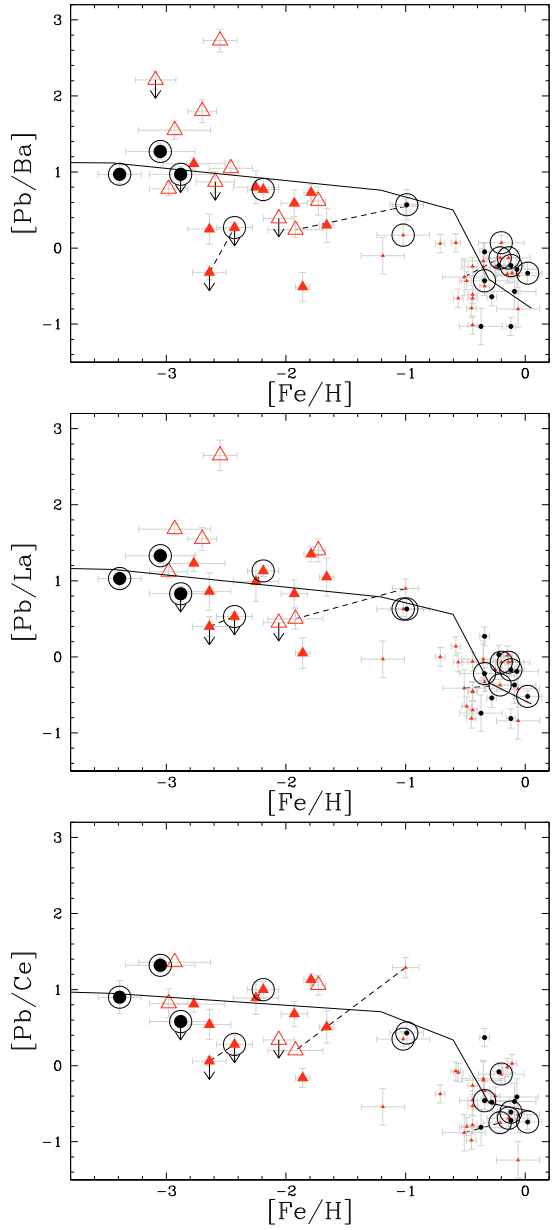

Fig. 6. Third-peak to second-peak $s$-process element ratios for CEMP-s and $\mathrm{Ba}$ stars (see Fig. 2 for a description of symbols). We also plot here our $\mathrm{Pb}$ measurements of CEMP-low-s stars, as these are the only available ones in the literature for this category. The solid line connects the predictions for different metallicities from Goriely \& Mowlavi (2000) (after 10 dredge-ups), Goriely \& Siess (2001), and Masseron et al. (2006).

or from the proton mixing profile has an impact of \pm 0.2 dex on the $\mathrm{Pb} / \mathrm{Ba}$ ratio. The scatter observed in Fig. 6 clearly indicates that additional parameters need to be considered. In particular, it 
has been suggested (Goriely \& Siess 2004) that the ingestion of protons in hot AGB stars can modify the neutron irradiation and lead to noticeably different s-abundance distributions. A deep hot third dredge-up tends to reduce the $s$-process efficiency, the protons injected into the $\mathrm{C}$-rich layers producing a ${ }^{14} \mathrm{~N}$-rich region rather than a ${ }^{13} \mathrm{C}$-rich pocket.

\section{The possible nature of the companions to CEMP-rs stars}

The puzzling abundance pattern in CEMP-rs stars, characterised by large overabundances of neutron-capture elements (as illustrated in Fig. 1), has given rise to various hypotheses (see Jonsell et al. 2006, for a detailed review). The binarity of these stars has now been confirmed (e.g., Paper II, and Barbuy et al. 2005). Because of the relatively old age of these low-metallicity halo stars, an initial pollution of their original gas by $\mathrm{C}, \mathrm{N}$, and neutron-capture elements requires a very rapidly evolving object. However, current models of massive stars predict a pure $r$-process pattern (Woosley \& Weaver 1995) or a weak $s$-process (Pignatari et al. 2008), but none support a rs pattern (and in particular the large $\mathrm{Pb}$ enhancement).

In addition, Jonsell et al. (2006) noticed that the large number of CEMP-rs stars observed at low metallicities casts doubt on the probability of a two-source pollution scenario including at least one massive star. However, neither the IMF nor the multiple system frequency are known at low metallicity. The estimates from Tumlinson (2007) indeed infer that the IMF should be peaked toward high masses at low metallicities, and the simulation of Vanhala \& Cameron (1998) predicts that the explosion of the first generations of massive stars would favour the formation of binaries, thus would privilege the formation of binary systems with the imprints of a massive star. Alternatively, Cohen et al. (2003) hypothesised that CEMP-rs stars are first polluted by the $s$-process from an AGB primary companion, which subsequently turns into a white dwarf. This white dwarf later accretes material back from the secondary companion, and if the white dwarf is an $\mathrm{O}-\mathrm{Ne}-\mathrm{Mg}$ dwarf, accretion-induced collapse of the white dwarf ensues and leads to a neutron star (Nomoto \& Kondo 1991; Justham et al. 2009). A neutrino-driven wind from the forming neutron star enriches the secondary star in the $r$ process elements, leading to the final abundances in the CEMPrs star. We emphasise that this scenario involves an $\mathrm{O}-\mathrm{Ne}-\mathrm{Mg}$ white dwarf, and this requirement strongly reduces the frequency of occurrence of the scenario.

Nevertheless, the calculations of Aoki et al. (2006) established that the $s$-process and $r$-process are almost independent contributors to the final yields. Following this argument, Johnson $\&$ Bolte (2004) failed to reproduce in detail the extensive abundance pattern observed in the CEMP-rs star CS 31062-50 by adding an $s$-process pattern to an $r$-process pattern. Furthermore, Masseron (2006) demonstrated that the addition of $\mathrm{Ba}$ and $\mathrm{Eu}$ abundances as observed in CEMP-s stars (representing the contribution of a low-metallicity AGB star) to the Ba and Eu abundance as observed in rII stars (representing the contribution of a low-metallicity massive star) falls below the amount of $\mathrm{Ba}$ and Eu observed in CEMP-rs stars. Hence, we argue that the double enhancement scenario does not hold. Thus, it appears that a non-standard $s$-process is the most suitable candidate for explaining the CEMP-rs phenomenon and most certainly from a unique companion (likely an AGB star).
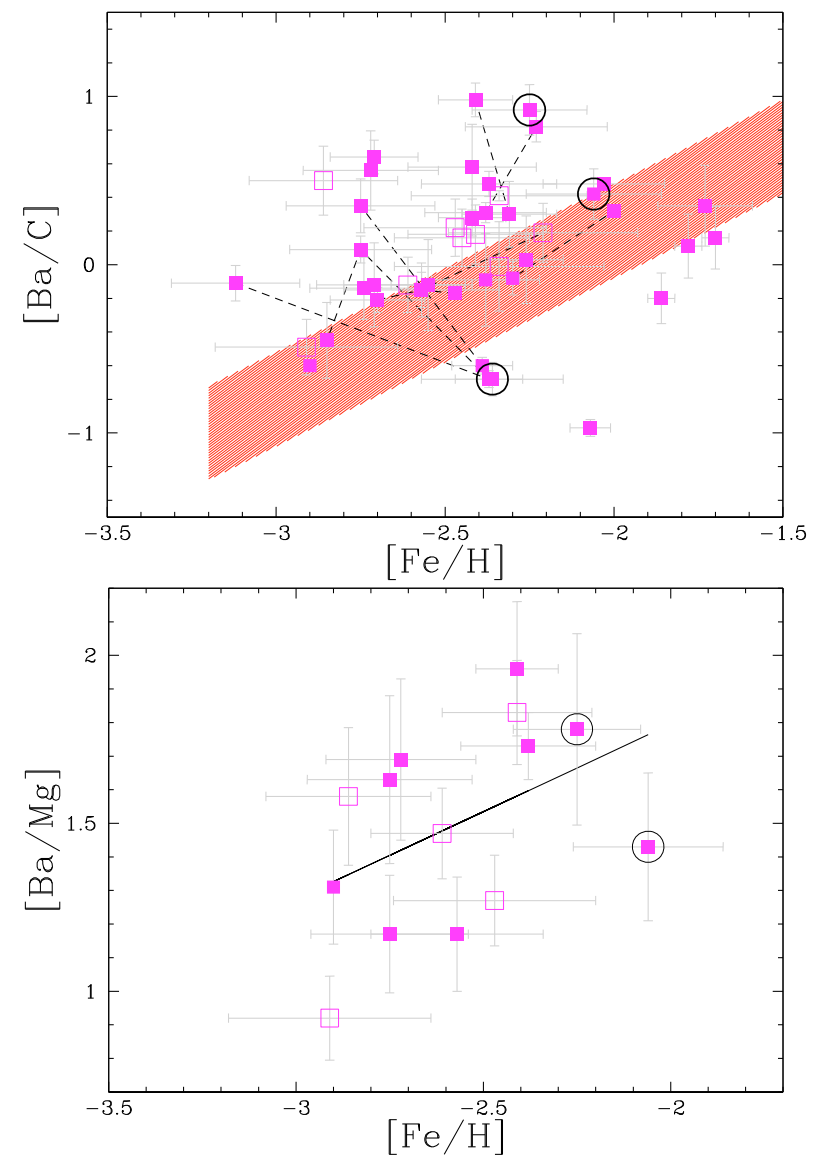

Fig. 7. Upper panel: $[\mathrm{Ba} / \mathrm{C}]$ in CEMP-rs stars as a function of metallicity (see Fig. 2 for a description of symbols). No correlation is observed, in contrast to CEMP-s stars (shaded area from Fig. 5). Lower panel: $[\mathrm{Ba} / \mathrm{Mg}]$ in CEMP-rs stars with $[\mathrm{Mg} / \mathrm{Fe}]>0.6$, as a function of metallicity. There is an apparent trend (the correlation coefficient of the least squares fit (solid line) is 0.55 ).

\subsection{Evidence of the operation of the ${ }^{22} \mathrm{Ne}(\alpha, n)^{25} \mathrm{Mg}$ neutron source in CEMP-rs companions}

In Sect. 5, we established that in CEMP-s stars, both the $s$ process element overabundances (reflected in $\mathrm{Ba} / \mathrm{C}$ ) and the $s$ process efficiency (reflected in the third-peak to second-peak abundance ratios) depended, at least to some extent, on the metallicity of the AGB star. One striking result for CEMP-rs stars is that the $\mathrm{Ba} / \mathrm{C}$ ratio does not show any correlation with metallicity (Fig. 7), despite the strong correlation between the production of $\mathrm{Ce}$ and metallicity highlighted by the small scatter in $[\mathrm{Ce} / \mathrm{Fe}]$ values in CEMP-rs stars (Fig. 8). Similarly, the $s$-process efficiency does not show any correlation with metallicity but rather with $\mathrm{N}$ (Fig. 9)!

According to Goriely \& Siess (2005), the correlation between $[\mathrm{Pb} / h s]$ (where $h s=\mathrm{Ba}, \mathrm{La}$, or $\mathrm{Ce}$ ) and $\mathrm{N}$ is evidence that a convective $s$-process driven by the ${ }^{22} \mathrm{Ne}(\alpha, \mathrm{n})^{25} \mathrm{Mg}$ neutron source operates in thermal pulses of AGB stars. When thermal pulse occurs, $\mathrm{N}$ (left over from the former hydrogen-burning) is fully burnt by the $\left.{ }^{14} \mathrm{~N}(\alpha, \gamma){ }^{18} \mathrm{~F}\left(\beta^{+}, v\right){ }^{18} \mathrm{O}(\alpha, \gamma)\right)^{22} \mathrm{Ne}$ reaction, and neutrons are released by the subsequent ${ }^{22} \mathrm{Ne}(\alpha, \mathrm{n})^{25} \mathrm{Mg}$ reaction. When the temperature is high enough in the convective pulse (i.e., the AGB star is sufficiently massive), this reaction is a very efficient neutron source leading to an efficient production of $s$-process elements. This is consistent with the large overabundances of $\mathrm{Ba}, \mathrm{La}, \mathrm{Ce}$, and $\mathrm{Pb}$ observed in CEMP-rs stars. It 

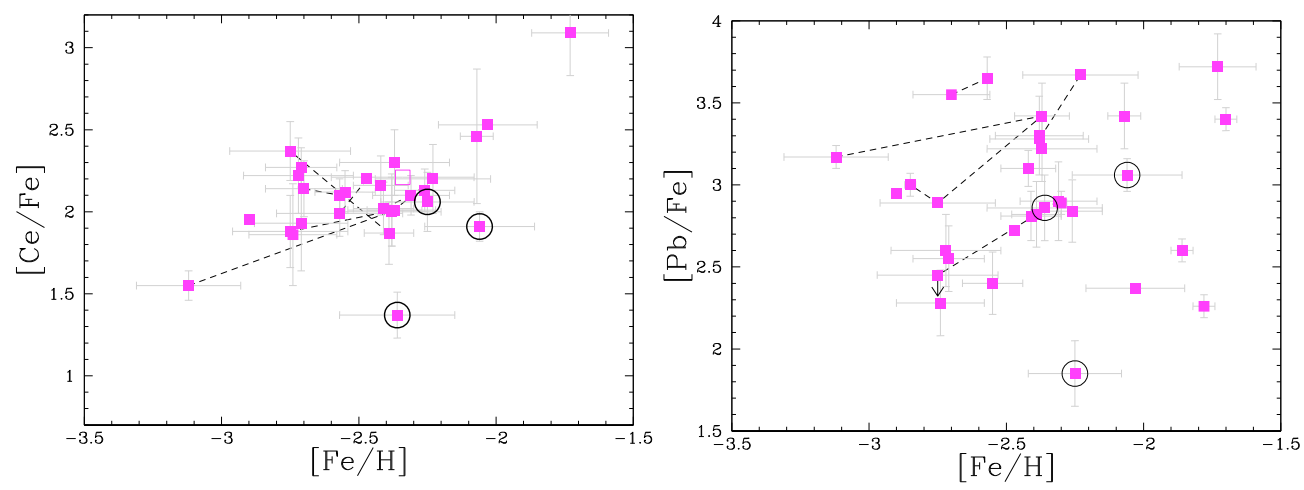

Fig. 8. $\mathrm{Ce}$ and $\mathrm{Pb}$ enhancements as a function of metallicity in CEMP-rs stars (see Fig. 2 for a description of symbols). The narrow scatter in $\mathrm{Ce} / \mathrm{Fe}$ highlights a strong dependence of $\mathrm{Ce}$ production on metallicity in contrast to $\mathrm{Pb}$, also produced in large quantities but with a wide range of abundances. If dilution cause the scatter in $[\mathrm{Pb} / \mathrm{Fe}]$, we would expect a similar scatter in $[\mathrm{Ce} / \mathrm{Fe}]$.
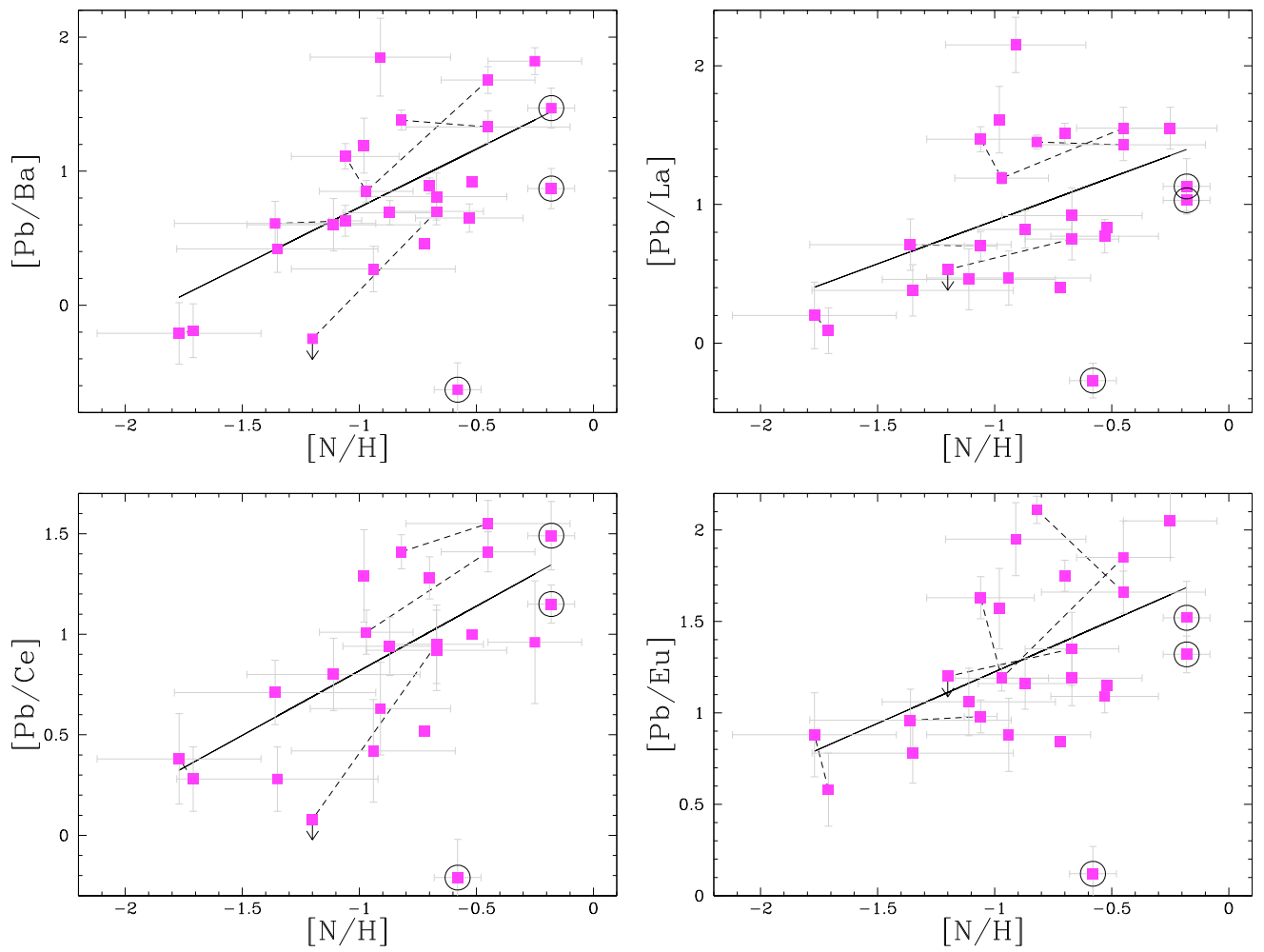

Fig. 9. Third- to second-peak abundance ratios as a function of $\mathrm{N}$ in CEMP-rs stars (see Fig. 2 for a description of symbols). Solid lines are least squares fits. The corresponding correlation coefficients from top-left to down-right panels are $0.65,0.51,0.68$, and 0.58 . Clear correlations are observed between third- and second-peak abundance ratios, especially in the case of $\mathrm{Ce}$.

is also expected that ${ }^{25} \mathrm{Mg}$ and ${ }^{26} \mathrm{Mg}$ are produced significantly in AGB stars with high masses (Karakas \& Lattanzio 2003). This is what is possibly being observed in Fig. 10, where the observed $\mathrm{Mg}\left(={ }^{24} \mathrm{Mg}+{ }^{25} \mathrm{Mg}+{ }^{26} \mathrm{Mg}\right)$ is enhanced in some of the CEMP-rs stars.

The high neutron density associated with ${ }^{22} \mathrm{Ne}(\alpha, \mathrm{n})^{25} \mathrm{Mg}$ is also predicted to favour the production of so-called sr-nuclei (Gallino et al. 1998; Goriely \& Mowlavi 2000), notably ${ }^{142} \mathrm{Ce}$, as well as $r$-process nuclei such as ${ }^{151} \mathrm{Eu}$ and ${ }^{153} \mathrm{Eu}$ (Goriely \& Siess 2005). It then becomes clear why $[\mathrm{Eu} / \mathrm{Fe}]$ increases at the same time as $[\mathrm{Ba} / \mathrm{Fe}]$ (Fig. 1).

If the ${ }^{22} \mathrm{Ne}(\alpha, \mathrm{n})^{25} \mathrm{Mg}$ neutron source is responsible for the synthesis of the heavy elements now observed in CEMP-rs stars, $[\mathrm{Ba} / \mathrm{Mg}]$ (rather than $[\mathrm{Ba} / \mathrm{C}]$, as in the case of CEMP-s stars) should correlate with metallicity. The right panel of Fig. 7 provides some evidence of this trend, although the scatter is large. The causes of this scatter are numerous: (i) the Mg abundance may be dominated by the isotope ${ }^{24} \mathrm{Mg}$, not altered by the operation of ${ }^{22} \mathrm{Ne}(\alpha, \mathrm{n})^{25} \mathrm{Mg}$; and (ii) the efficiency of the ${ }^{22} \mathrm{Ne}(\alpha, \mathrm{n}){ }^{25} \mathrm{Mg}$ neutron source is very sensitive to the temperature at the base of the thermal pulse, which is in turn a function of mass, metallicity, and pulse number. The lifetime of ${ }^{22} \mathrm{Ne}$ relative to ${ }^{22} \mathrm{Ne}(\alpha, \mathrm{n})^{25} \mathrm{Mg}$ (which controls the $s$-process efficiency) is also difficult to predict with certainty, given the large uncertainties that still affect the modelling of this reaction rate. To activate the ${ }^{22} \mathrm{Ne}(\alpha, \mathrm{n})^{25} \mathrm{Mg}$ reaction, temperatures higher than about $3.5 \times 10^{8} \mathrm{~K}$ are required at the base of the convective pulse. These temperatures are only expected in stars more massive than about $3 M_{\odot}$.

Figure 11 shows that $[\mathrm{La} / \mathrm{Ce}] \approx 0$ in CEMP-rs stars, while the calculations by Goriely $\&$ Siess (2005) predict a negative value 


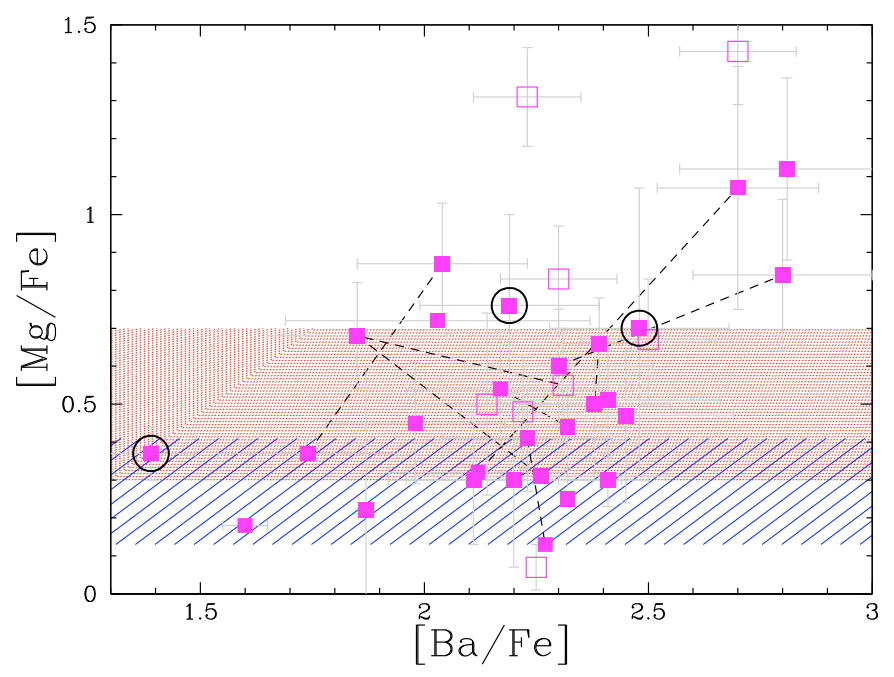

Fig. 10. $\mathrm{Mg}$ enhancement versus Ba enhancement in CEMP-rs stars (see Fig. 2 for a description of symbols). The red shaded area represents the average $[\mathrm{Mg} / \mathrm{Fe}] \pm 1 \sigma$ for CEMP-s stars, while the blue hatched area represent the average $[\mathrm{Mg} / \mathrm{Fe}] \pm 1 \sigma$ for rI and rII stars reflecting the Galactic mean value. $\mathrm{Mg}$ and $\mathrm{Ba}$ are simultaneously enhanced in some CEMP-rs stars compared to the Galactic mean value, reinforcing the idea that the ${ }^{22} \mathrm{Ne}(\alpha, \mathrm{n})^{25} \mathrm{Mg}$ reaction occurred intensively in these stars.

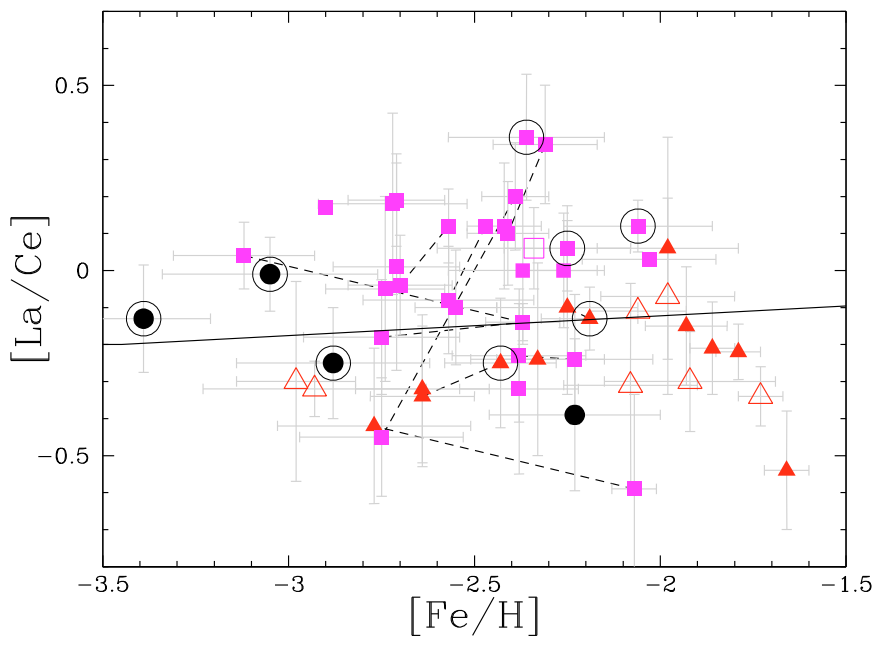

Fig. 11. $[\mathrm{La} / \mathrm{Ce}]$ in CEMP-s stars (red triangles) and in CEMP-rs stars (magenta squares). The solid line connects the predictions for different metallicities from Goriely \& Mowlavi (2000) (after 10 dredge-ups), Goriely \& Siess (2001) and Masseron et al. (2006). While CEMP-s stars show negative $[\mathrm{La} / \mathrm{Ce}]$ ratios in agreement with predictions, CEMP-rs show $[\mathrm{La} / \mathrm{Ce}] \approx 0$ ratios.

from the operation of the ${ }^{13} \mathrm{C}(\alpha, \mathrm{n}){ }^{16} \mathrm{O}$ neutron source. The consistently larger [La/Ce] values observed for CEMP-rs stars are thus a strong indication that the ${ }^{13} \mathrm{C}(\alpha, \mathrm{n}){ }^{16} \mathrm{O}$ neutron source does not operate in those stars, as already suggested above from various other arguments. It is also meaningful that the [La/Ce] values observed in CEMP-rs stars are compatible with the values $0.2-0.4$ dex predicted from the operation of the ${ }^{22} \mathrm{Ne}(\alpha, \mathrm{n})^{25} \mathrm{Mg}$ neutron source in warm pulses, and after dilution in the AGB envelope (Goriely \& Siess 2005). The operation of ${ }^{22} \mathrm{Ne}(\alpha, \mathrm{n}){ }^{25} \mathrm{Mg}$ alone does not, however, lead to $\mathrm{Pb}$ production, and would thus appear inconsistent with the large $[\mathrm{Pb} / \mathrm{hs}]$ ratios seen in Fig. 9. Fortunately, such large $\mathrm{Pb}$ overabundances are predicted when ${ }^{13} \mathrm{C}(\alpha, \mathrm{n}){ }^{16} \mathrm{O}$ and ${ }^{22} \mathrm{Ne}(\alpha, \mathrm{n}){ }^{25} \mathrm{Mg}$ operate jointly, which should be the case in a limited mass range

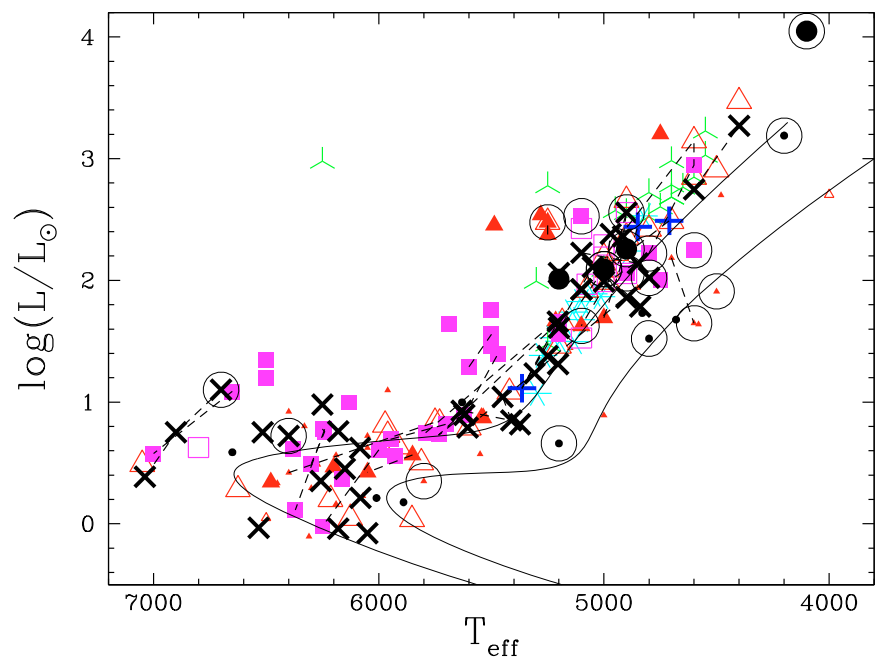

Fig. 12. The Hertzsprung-Russell diagram for the entire sample (see Fig. 2 for a description of symbols). The luminosity has been calculated with the following formula: $\log L / L_{\odot}=\log \left(M / M_{\odot}\right)+4 \log \left(T_{\text {eff }} / T_{\text {eff } \odot}\right)-$ $\log \left(g / g_{\odot}\right)$, adopting $0.8 M_{\odot}$ for all the stars and taking $T_{\text {eff }}$ and $\log (g)$ from Col. (2) of Tables 1 and 3. The black solid lines are $12 \mathrm{Gyr}$ isochrones for $[\mathrm{Fe} / \mathrm{H}]=-1.01$ (right curve) and -2.31 (left curve), both $\alpha$-enhanced, from VandenBerg et al. (2006). While most of the stars fall on the isochrones, some CEMP-rs stars (magenta squares) and a couple of CEMP-no stars (black crosses) are bluer and more luminous.

(Goriely \& Siess 2004, 2005). Finally, we emphasise that the latest up-to-date models of Cristallo et al. (2009) support a non-negligible contribution of the ${ }^{22} \mathrm{Ne}(\alpha, \mathrm{n})^{25} \mathrm{Mg}$ reaction to the $s$-process in a $2 M_{\odot}$ low-metallicity AGB star.

\subsection{Evidence of mixing in CEMP-rs stars}

It must be emphasised that the evolution in the CEMP star itself could also affect the abundance ratios such as $[\mathrm{X} / \mathrm{Fe}]$ or $[\mathrm{X} / \mathrm{H}]$, and even the $[\mathrm{C} / \mathrm{N}]$ ratio. After stars leave the main sequence and before they ascend the giant branch, they undergo in general the first dredge-up. This convective process dredges up material that has been processed by the $\mathrm{CN}$ cycle during the main sequence, and brings N-rich and C-poor material to the surface. Therefore, in normal stars that have homogeneous envelopes, the first dredge-up is responsible for low $[\mathrm{C} / \mathrm{H}]$ and high $[\mathrm{N} / \mathrm{H}]$ above some luminosity threshold $\left(\log L / L_{\odot} \sim 1\right)$.

However, the situation may differ from the above picture describing the standard first dredge-up, if a star has transferred AGB material in a thin layer on its surface while on the main sequence, as CEMP stars do. The dilution resulting from mass transfer is governed by the convective or radiative nature of the accretor envelope, and by the difference between the molecular weights of the accretor's initial envelope and the accreted matter. When the abundance differences between the initial composition and the accreted material are large enough, thermohaline mixing operates (Proffitt \& Michaud 1989; Barbuy et al. 1992), and dilutes the accreted material in the star's envelope before the first dredge-up occurs. The respective depths of thermohaline mixing and first dredge-up fixes the surface $[\mathrm{C} / \mathrm{H}]$ and $[\mathrm{N} / \mathrm{H}]$ ratios. If thermohaline mixing does not extend very deeply, dilution in the star's envelope by the first dredge-up dominates and all abundances of accreted elements (e.g., [C/H], [N/H], [Ba/Fe]) decline sharply (Denissenkov \& Pinsonneault 2008). When thermohaline mixing is sufficiently deep, the first dredge-up may not even leave any observable signature on the $\mathrm{C}$ and $\mathrm{N}$ abundances. In 

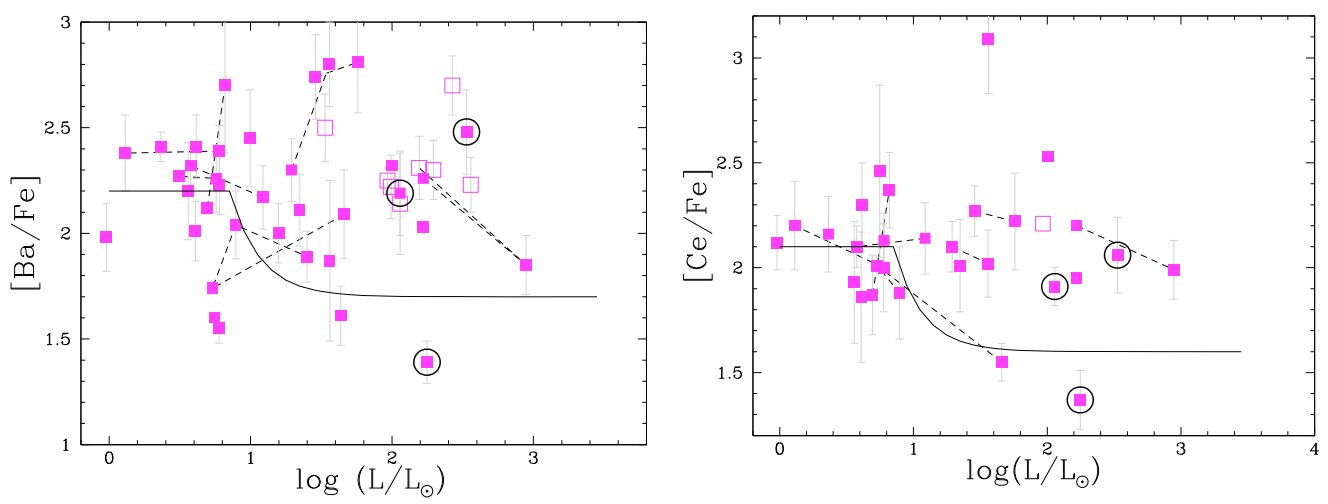

Fig. 13. $[\mathrm{Ba} / \mathrm{Fe}]$ and $[\mathrm{Ce} / \mathrm{Fe}]$ versus luminosity in CEMP-rs stars (see Fig. 2 for a description of symbols). Whereas the accreted $[\mathrm{Ba} / \mathrm{Fe}]$ is expected to be diluted in the star after the first dredge-up $\left(\log \left(L / L_{\odot}\right) \approx 1\right)$ as predicted by Denissenkov \& Pinsonneault (2008) (solid line), $[\mathrm{Ba} / \mathrm{Fe}]$ in CEMP-rs stars does not show any such signature of depletion between main sequence and giant stars $\left(\langle[\mathrm{Ba} / \mathrm{Fe}]\rangle_{\mathrm{dwarfs}}=2.23 \pm 0.39\right.$ and $\left.\langle[\mathrm{Ba} / \mathrm{Fe}]\rangle_{\text {giants }}=2.21 \pm 0.34\right)$. The $[\mathrm{Ce} / \mathrm{Fe}]$ scatter is very small $(\sigma=0.24$ dex compared to 0.19 dex random measurement uncertainty $)$ and independent of the star luminosity.
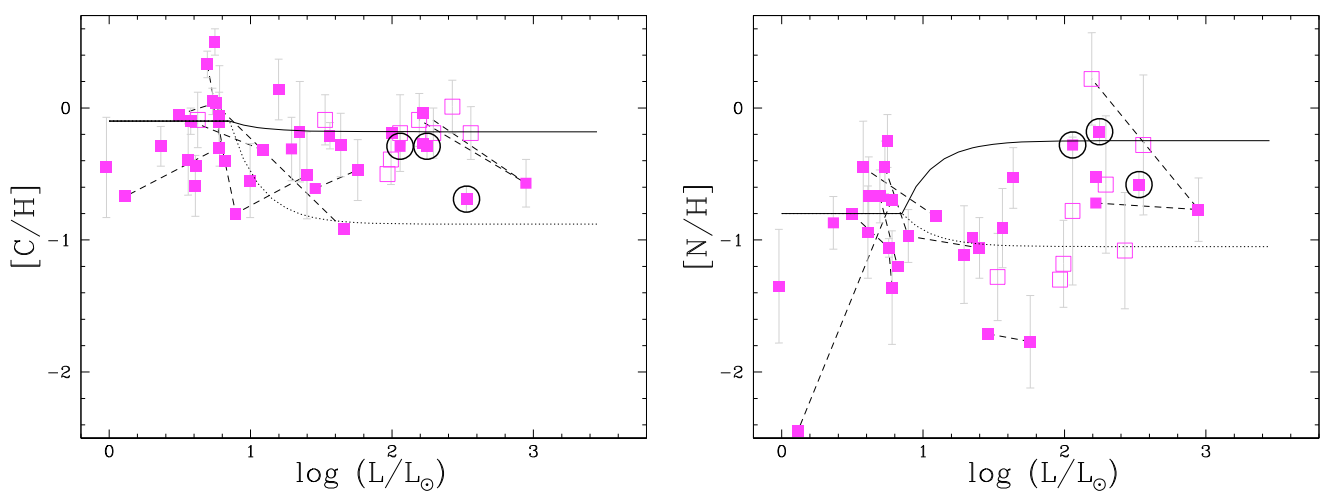

Fig. 14. $\mathrm{C}$ and $\mathrm{N}$ abundances in CEMP-rs stars (see Fig. 2 for a description of symbols). The solid line is the predicted abundance trend with thermohaline mixing, while the dotted line is without thermohaline mixing but dilution after the first dredge-up from Stancliffe et al. (2007). C is constant with luminosity $\left(\langle[\mathrm{C} / \mathrm{H}]\rangle_{\mathrm{dwarfs}}=-0.23 \pm 0.33\right.$ and $\left.\langle[\mathrm{C} / \mathrm{H}]\rangle_{\text {giants }}=-0.30 \pm 0.24\right)$. However, it is unclear whether $\mathrm{N}$ is enhanced after the 1st dredge-up. We note as well that the $\mathrm{C}$ scatter is much lower than the $\mathrm{N}$ scatter.

the extreme case where thermohaline mixing drags the accreted $\mathrm{C}$ and $\mathrm{N}$ into the $\mathrm{H}$ burning region, $\mathrm{C}$ is processed through the $\mathrm{CN}$ cycle. The first dredge-up is then expected to bring to the surface both depleted $\mathrm{C}$ and enhanced $\mathrm{N}$ (Stancliffe et al. 2007), and leave the other ratios unchanged (e.g., $[\mathrm{Ba} / \mathrm{Fe}],[\mathrm{C}+\mathrm{N} / \mathrm{H}]$ ).

In addition to placing constraints on the nucleosynthesis processes in CEMP companions, we can use abundance ratios to study the depth of the dilution in the polluted star by comparing abundance ratios at different evolutionary stages. We emphasise that this approach is only valid when the overabundances of accreted material are significantly higher than the initial abundances. In this regard, CEMP-rs stars offer the best conditions because they have the largest overabundances. We determined the luminosities of our sample stars from their surface parameters $\left(T_{\text {eff }}\right.$ and $\left.\log g\right)$ and assumed that they are all $0.8 M_{\odot}$ stars (see Fig. 12).

In Figs. 13 and $14 \mathrm{C}$ abundances, $[\mathrm{Ba} / \mathrm{Fe}]$, and $[\mathrm{Ce} / \mathrm{Fe}]$ ratios do not vary significantly between dwarfs and giants. Therefore, we see no signature of dilution in CEMP-rs stars, so that the accreted material must have been mixed in the star during the main sequence and/or turn off.

The accretion of material, particularly on the main sequence, may affect the position of a star in the HR diagram. In Fig. 12, it is remarkable that all stars agree well with a 12 Gyr isochrone, except for some of the main-sequence and turn-off CEMP-rs stars, which are significantly bluer. One should keep in mind, however, that in some studies, isochrones were used to determine gravities. The off-sequence stars occupy a similar region of the HR diagram as the blue stragglers, where mixing has occurred on the main sequence. In their model, Stancliffe et al. (2007) found that thermohaline mixing of the C-rich material is deep enough to reach the $\mathrm{H}$ combustion layers, so that it stimulates $\mathrm{H}$ burning and increases the luminosity of the star. Jonsell et al. (2006) already suspected a relation between CEMP-rs stars and blue stragglers (their hypothesis VIII). According to Stancliffe et al. (2007), the additional luminosity is a direct consequence of the enhanced $\mathrm{CN}$ mixed throughout the star, which thus boosts the $\mathrm{CNO}$ cycle. Therefore, they also expect strong $\mathrm{N}$ enhancement after the 1st dredge-up (occurring around $\log \left(L / L_{\odot}\right) \approx 1$ ). However, Fig. 14 shows no clear increase in $\mathrm{N}$ for stars with $\log \left(L / L_{\odot}\right)>1$.

The examination of the abundances as a function of CEMP-rs parameters leads to 3 main conclusions:

(i) the abundances observed in CEMP-rs stars are unaffected by the dilution associated with the first dredge-up in the atmosphere of the CEMP star;

(ii) in contrast, the accreted material seems to alter the evolution of CEMP-rs stars, as for blue stragglers. This requires a large amount of accreted material, which is consistent with the companion losing a large amount of mass, thus being relatively massive; 
T. Masseron et al.: A holistic approach to carbon-enhanced metal-poor stars
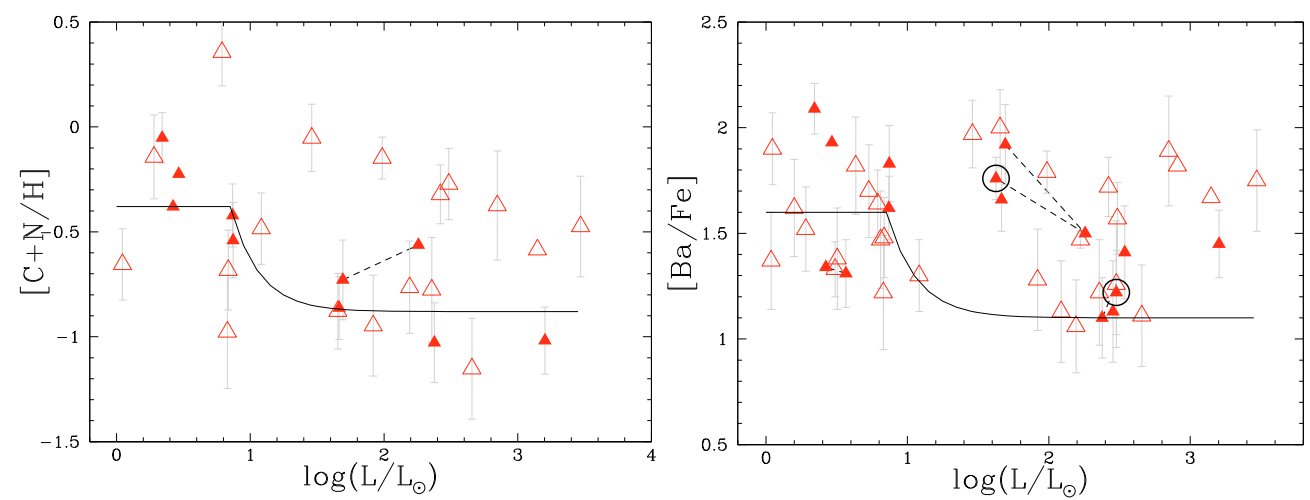

Fig. 15. $[\mathrm{C}+\mathrm{N} / \mathrm{H}]$ and $[\mathrm{Ba} / \mathrm{Fe}]$ in CEMP-s stars as a function of luminosity. While the average $[\mathrm{C}+\mathrm{N} / \mathrm{H}]$ shows a discrepancy between dwarfs and giants $(\langle[\mathrm{C}+\mathrm{N} / \mathrm{H}]\rangle=-0.38 \pm 0.35$ for dwarfs and $-0.64 \pm 0.32$ for giants $)$ in apparent agreement with the predictions of Denissenkov \& Pinsonneault (2008) (solid line), $[\mathrm{Ba} / \mathrm{Fe}]$ is very homogeneous between dwarfs and giants $(\langle[\mathrm{Ba} / \mathrm{Fe}]\rangle=1.57 \pm 0.25$ for dwarfs and $1.51 \pm 0.30$ for giants).

(iii) the variation in the amount of transferred material from one CEMP-rs star to another is below the measurement uncertainties (right panel of Fig. 13). Therefore, abundance trends such as that observed for $\mathrm{N} / \mathrm{H}$ are free from the scatter associated with variable mass-transfer efficiencies.

\subsection{Thermohaline mixing and first dredge-up dilution in CEMP-s and CEMP-no stars}

As for CEMP-rs stars, CEMP-s stars exhibit large C enhancements in their atmosphere; therefore, thermohaline mixing could also be expected to be operating. Aoki et al. (2008) point out that their earlier data (Aoki et al. 2007) for Ba-enhanced CEMP stars do not support thermohaline mixing. We confirm that in CEMP$\mathrm{s}$ stars, there is an apparent discrepancy in the average $[\mathrm{C}+\mathrm{N} / \mathrm{H}]$ of giants and dwarfs, but $[\mathrm{Ba} / \mathrm{Fe}]$ remains remarkably homogeneous (Fig. 15). However, the dilution should affect the abundances of the accreted elements by an identical factor. Since this is not what is observed in CEMP-s stars from the comparison of $\mathrm{C}+\mathrm{N}$ and $\mathrm{Ba}$ data, we conclude that there is no clear signature of dilution of the accreted material after the first dredge-up in CEMP-s stars, in contrast to the conclusions of Aoki et al. (2008).

For CEMP-no stars, the situation is different. As noted by Aoki et al. (2002c), CEMP-no stars exhibit on average mild Cenrichment: CEMP-no stars have $[\mathrm{C} / \mathrm{H}]=-1.42 \pm 0.86$, whereas CEMP-rs have $[\mathrm{C} / \mathrm{H}]=-0.28 \pm 0.28$ and CEMP-s stars have $[\mathrm{C} / \mathrm{H}]=-0.62 \pm-0.34$. Because thermohaline mixing depends on the molecular weight gradient induced by the accretion of heavy elements, a less efficient thermohaline mixing than the one in CEMP-rs stars must be expected for CEMP-no stars. Lucatello et al. (2006) and Aoki et al. (2007) do find a trend of $[\mathrm{C}+\mathrm{N} / \mathrm{H}]$ with the CEMP luminosity. Hence, these two works conclude that thermohaline mixing was negligible and that what is seen is the dilution of the accreted material by the first dredgeup, as estimated by Denissenkov \& Pinsonneault (2008). We note however that Lucatello et al. (2006) do not separate CEMP classes, so the trend they found is certainly smoothed between CEMP-no, CEMP-s, and CEMP-rs stars.

We show in Fig. 16 that there is a correlation between metallicity and luminosity in CEMP-no stars. In surveys, more giants than dwarfs are expected to be found since their high luminosity allows us to detect them in a larger volume. Consequently, it would appear natural that the more the metallicity decreases and the fewer metal-poor stars that are detected (e.g., Ryan \& Norris 1991), the more the frequency of giants increases, leading to an apparent correlation between metallicity and luminosity (see Masseron et al. 2006, for more detailed estimates). Therefore, we argue that the conclusions of Lucatello et al. (2006) and Aoki et al. (2007) are observationally biased so that the decrease in $[\mathrm{C}+\mathrm{N} / \mathrm{H}]$ as a function of luminosity is dominated by the metallicity trend. Thus, we conclude that with the current data we cannot measure the impact of thermohaline mixing in CEMP-no stars.

\subsection{Comparison between CEMP-s and CEMP-rs stars}

After examining the neutron-capture elements in CEMP-rs stars, we compare in this section light-element abundances in CEMP-s and CEMP-rs to compare the properties of their respective companions.

\subsubsection{Carbon and nitrogen}

$\mathrm{N}$ is generally thought to be produced by the $\mathrm{CN}$ cycle. As a consequence of this cycle, ${ }^{14} \mathrm{~N}$ increases at the expense of ${ }^{12} \mathrm{C}$, and ${ }^{12} \mathrm{C} /{ }^{13} \mathrm{C}$ is lowered from its initial value. However, in lowand intermediate-mass metal-poor stars, ${ }^{14} \mathrm{~N}$ can be produced at many different stages, notably after the first dredge-up, during the RGB phase by means of "extra mixing" (e.g., Gilroy 1989; Boothroyd \& Sackmann 1999) and during the AGB phase if HBB occurs at the bottom of the convective envelope or if there is "extra mixing" below the convective envelope, of a similar nature than the one occurring during the RGB, but called Cool-Bottom Processing (CBP) in the framework of AGB evolution (Abia et al. 2001; Nollett et al. 2003). For CEMP stars, the situation is thus more complex since both the CEMP star and its companion may have undergone $\mathrm{CN}$ processing. Based on the observation that CEMP stars are also N-rich stars, it is commonly admitted that $\mathrm{N}$ originates in the same source as $\mathrm{C}$, i.e. its companion. In our sample, very few CEMP stars have high enough luminosities $\left(\log \left(L / L_{\odot}\right) \gtrsim 2.2\right)$ to undergo extra mixing (Spite et al. 2005), and a fortiori neither HBB nor CBP. Thus, CEMP stars could not have produced $\mathrm{N}$ by these processes. Moreover, we have demonstrated in Sect. 6.2 that there is no significant enhancement of $\mathrm{N}$ after CEMP stars' first dredgeup. Finally, there are strong arguments in favour of $\mathrm{N}$ originating in the former AGB companion, notably that the abundances of 

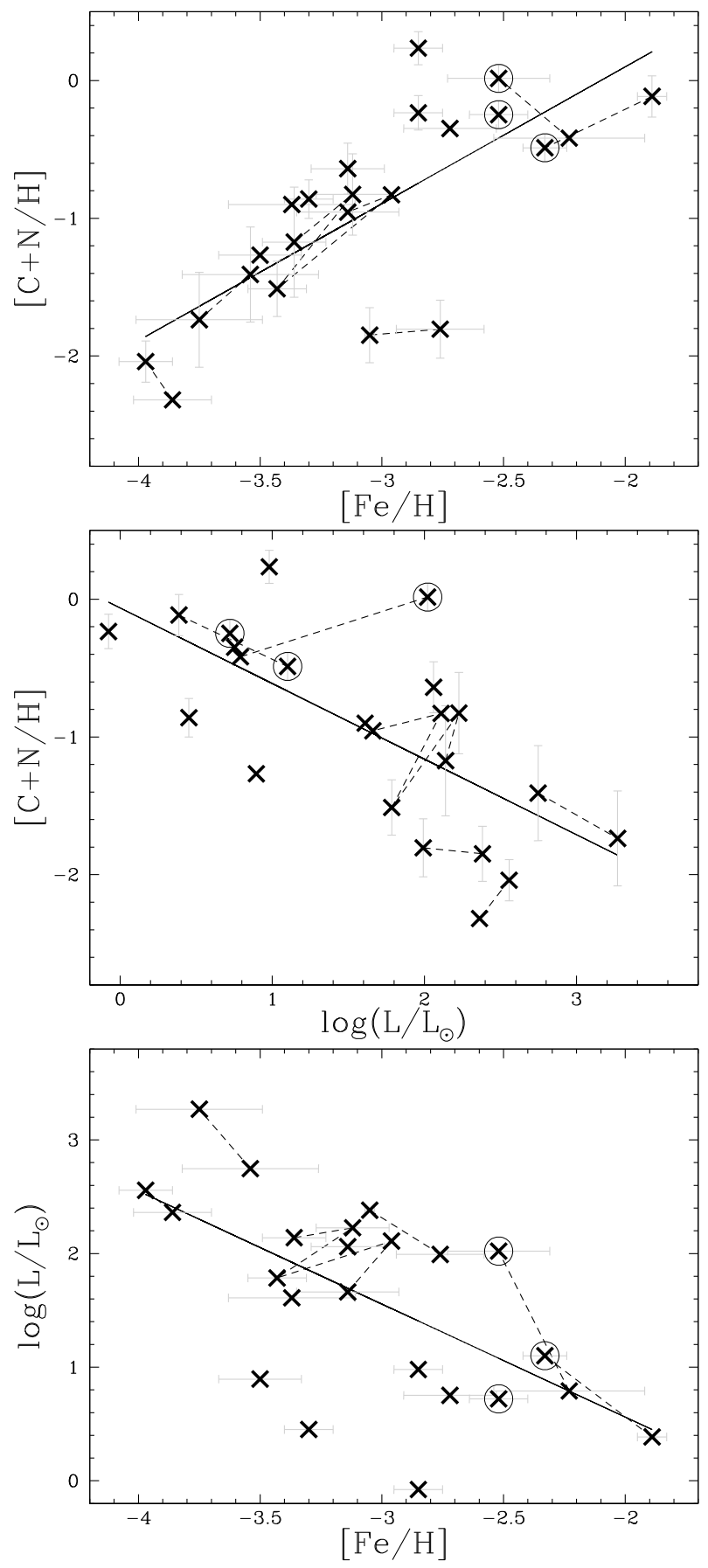

Fig. 16. $[\mathrm{C}+\mathrm{N} / \mathrm{H}]$ in CEMP-no stars as a function of metallicity (upper left) and luminosity (upper right). There is a correlation in both cases (the solid lines represent the least squares fits), implying that luminosity is anti-correlated with metallicity (lower panel). This highlights an observational bias in CEMP-no stars.

the $s$-process elements in CEMP-rs stars are correlated with $\mathrm{N}$ (Fig. 9). Since it is very unlikely that CEMP stars have synthesised any $s$-process elements in their interiors, their AGB companions must have produced both the heavy elements and all the observed $\mathrm{N}$ (as well as ${ }^{12} \mathrm{C}$ and ${ }^{13} \mathrm{C}$ ).

The model predictions displayed in Fig. 17 indicate that intermediate-mass AGB stars with $\mathrm{HBB}$ should have low $[\mathrm{C} / \mathrm{N}]$ and low ${ }^{12} \mathrm{C} /{ }^{13} \mathrm{C}$ ratios. In contrast, for low-mass AGB stars, no $\mathrm{CN}$ processing is expected after the second dredge-up. Consequently, in those stars, the cumulative amount of ${ }^{12} \mathrm{C}$

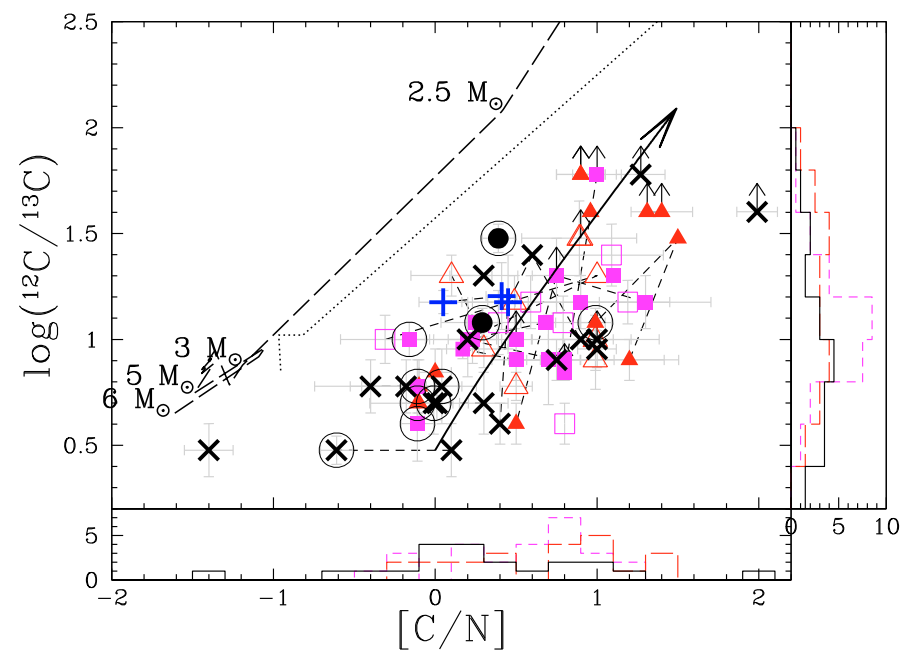

Fig. 17. ${ }^{12} \mathrm{C} /{ }^{13} \mathrm{C}$ as a function of $[\mathrm{C} / \mathrm{N}]$ ratios for CEMP stars (see Fig. 2 for symbols). The long-dashed line is the prediction for low-metallicity AGB stars from Karakas \& Lattanzio (2007) and the dotted line is the prediction from Herwig (2004). It is remarkable that all CEMP stars fall in the same region of this diagram. The black arrow represents the addition of an increasing amount of pure ${ }^{12} \mathrm{C}$, starting from a ${ }^{12} \mathrm{C} /{ }^{13} \mathrm{C}$ near the $\mathrm{CN}$ equilibrium value and $[\mathrm{C} / \mathrm{N}]=0$. No current models are able to predict the intermediate $\mathrm{C} / \mathrm{N}$ ratios observed in CEMP stars (see text). We note that CEMP-no stars have on average a slightly lower $[\mathrm{C} / \mathrm{N}]$ ratio and a lower ${ }^{12} \mathrm{C} /{ }^{13} \mathrm{C}$ than CEMP-s and CEMP-rs stars.

dredged-up during the AGB phase results in a high $[\mathrm{C} / \mathrm{N}]$ ratio and a high ${ }^{12} \mathrm{C} /{ }^{13} \mathrm{C}$ ratio. In contrast, all CEMP stars show relatively low ${ }^{12} \mathrm{C} /{ }^{13} \mathrm{C}$ ratios. This suggests that $\mathrm{CN}$ processing may have occurred between 3rd dredge-ups, which are known to bring up large amounts of ${ }^{12} \mathrm{C}$ to the surface. However, as already noticed by Johnson et al. (2007), CEMP stars have C/N ratios that are intermediate between the high ratios expected in low-mass stars and the low ratios expected in intermediate-mass stars with $\mathrm{HBB}$. A low ${ }^{12} \mathrm{C} /{ }^{13} \mathrm{C}$ ratio with $[\mathrm{C} / \mathrm{N}] \approx 0$ cannot be obtained by a complete $\mathrm{CN}$ cycle (which leads instead to ${ }^{12} \mathrm{C} /{ }^{13} \mathrm{C} \approx 4$ and $[\mathrm{C} / \mathrm{N}] \approx-1.3$, as does HBB). Therefore, as already concluded by Aoki et al. (2002c), C has only been partly processed (possibly in the H-burning shell) before it reaches the quiescent convective envelope. This is indeed a characteristic feature of CBP. We also show in Fig. 17 that there is a correlation between ${ }^{12} \mathrm{C} /{ }^{13} \mathrm{C}$ and $\mathrm{C} / \mathrm{N}$ in CEMP stars. It is remarkable that all observed CEMP stars are located in the same region of this diagram and follow the same trend. This correlation is well reproduced by varying the amount of pure ${ }^{12} \mathrm{C}$. Indeed, the observed trend might be ascribed to the competition between third dredge-up and CBP.

To summarise, although the mechanism responsible for the $\mathrm{N}$ production may be attributed to $\mathrm{CBP}$, no current AGB models reproduce the trend observed in Fig. 17. Thus, we cannot use $\mathrm{N}$ to place additional constraints on the mass of the progenitor. Finally, as long as the physical mechanism responsible for the CBP remains unidentified, the relation between the nitrogen and $s$-process productions in CEMP-rs stars is difficult to interpret because these two elements are supposedly produced in two distinct parts of the AGB star. Nevertheless, assuming that some common mechanism, such as rotation (Decressin \& Charbonnel 2006), thermohaline mixing (Cantiello \& Langer 2008), or Dual Shell Flashes (Campbell \& Lattanzio 2008), drives the CBP and the $s$-process, this correlation can be used to test these different models. 
T. Masseron et al.: A holistic approach to carbon-enhanced metal-poor stars

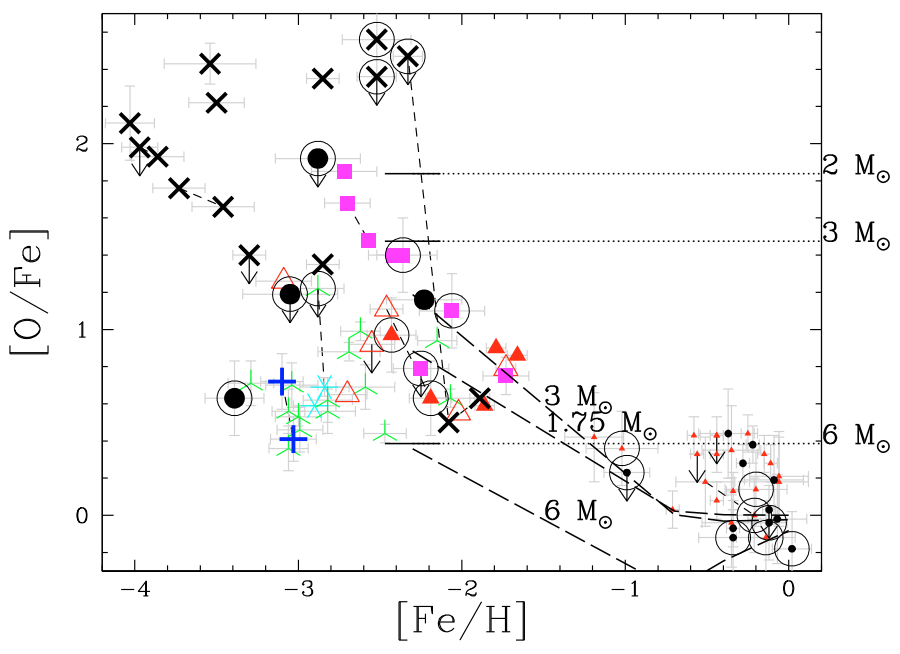

Fig. 18. O abundances in various classes of metal-poor stars (see Fig. 2 for a description of symbols). Dashed lines represent $\mathrm{O}$ predictions as a function of metallicity for different masses from Karakas \& Lattanzio (2007) and dotted lines point toward Herwig (2004) predictions for different masses (as indicated on the right-hand scale) at $[\mathrm{Fe} / \mathrm{H}] \approx-2.3$. O is more enhanced in CEMP-rs (magenta squares) than in CEMP-s stars (red triangles) with the same metallicity. We also notice that most of CEMP-no stars have even larger $\mathrm{O}$ enhancements. Note that none of the displayed $\mathrm{O}$ abundances have been corrected for 3D or non-LTE effects.

\subsubsection{Oxygen}

Oxygen is also expected to be enhanced in low-metallicity AGB stars, because of the hotter conditions in the He-burning shell [thus activating ${ }^{12} \mathrm{C}(\alpha, \gamma){ }^{16} \mathrm{O}$ ] and deeper dredge-ups than in solar-metallicity AGB stars (Herwig 2004; Karakas \& Lattanzio 2007). Figure 18 indeed shows that CEMP-rs stars are enhanced in $\mathrm{O}$. In this figure, we do not attempt to correct the $\mathrm{O}$ abundance for systematic effects because they come from different indicators ([OI], O triplet, and $\mathrm{OH}$ lines). Although NLTE and $3 \mathrm{D}$ effects are expected to reduce the $\mathrm{O}$ abundance, the corrections ( $\approx 0.2$ dex; Takeda 2003; García Pérez et al. 2006) are smaller than the actual $\mathrm{O}$ enhancement in CEMP-rs compared to the normal galactic content represented by rI and rII stars. In this figure, CEMP-s stars also show a slight $\mathrm{O}$ enhancement but in this case, uncertainties from non-LTE and 3D effects are not negligible compared to the observed enhancement so that no firm conclusion can be drawn.

It must be stressed that $\mathrm{O}$ yields predicted by different models do not agree with each other. Herwig (2004) finds that the dilution in the AGB envelope always dominates over the quantity of dredged-up matter, thus yielding lower $\mathrm{O}$ enhancements as mass increases. In contrast, Karakas \& Lattanzio (2007) found that there is competition between dilution and hotter conditions in the He shell boosting the operation of the ${ }^{12} \mathrm{C}(\alpha, \gamma){ }^{16} \mathrm{O}$ reaction as mass increases. Thus, they predict maximum $\mathrm{O}$ yields in their models at around $3 M_{\odot}$. Because of these uncertainties in the model predictions, on the basis of oxygen data alone, it is impossible to infer the mass of the former AGB companion of the CEMP-rs stars. Nevertheless, we have found evidence of the operation of ${ }^{22} \mathrm{Ne}(\alpha, \mathrm{n})^{25} \mathrm{Mg}$ in the He-flash-driven convective zone in the former AGB companions of CEMP-rs stars. This reaction requires high temperatures $\left(3 \times 10^{8} \mathrm{~K}\right)$, which are also more favourable for the ${ }^{12} \mathrm{C}(\alpha, \gamma){ }^{16} \mathrm{O}$ reaction. Therefore, it appears that the enhancement of $\mathrm{O}$ and the occurrence of ${ }^{22} \mathrm{Ne}(\alpha, \mathrm{n})^{25} \mathrm{Mg}$ in the companions of CEMP-rs stars are consistent with them having a more massive AGB companion than CEMP-s stars.
Furthermore, it is established that the ON cycle occurs on a longer timescale than the $\mathrm{CN}$ cycle so that $\mathrm{O}$ is marginally burnt by HBB. We demonstrated in Sect. 6.4.1 that the CN cycle occurring in the companion of the CEMP stars is incomplete and this implies that the ON cycle is certainly far from taking place in the companions of CEMP stars. Therefore we can reasonably conclude that the $\mathrm{O}$ enhancements observed in CEMP stars do not originate from H-burning. Most of the CEMP stars are not evolved enough to process the accreted material themselves, with possibly two exceptions, however: CS 22891-171 and CS 30322-023. CS 22891-171 is not enriched in O, but is very $\mathrm{N}$-rich and does not follow the $[\mathrm{Pb} / \mathrm{Ce}]$ versus $[\mathrm{N} / \mathrm{H}]$ trend (Fig. 9). Its luminosity places it either at the top of the RGB or in the early-AGB phase. It is thus possible that this star has processed its accreted $\mathrm{O}$ into $\mathrm{N}$ through the $\mathrm{ON}$ cycle. This phenomenon would also be a good explanation of the properties of the peculiar C-depleted, O-normal and N-super-rich star CS 30322-023, located at the tip of the AGB (Masseron et al. 2006).

\subsubsection{Statistics}

Finally, we emphasise that our hypothesis that CEMP-rs stars are formed solely by pollution from an AGB companion contradicts most of the scenarios proposed to date (see Jonsell et al. (2006) for a thorough discussion of these). Using our extensive database of CEMP stars, we discuss more thoroughly one of these scenarios, which invokes a double-pollution episode (typically from AGB stars and type-II supernovae), one contributing to the $s$-process enhancement and the other to the $r$-process enhancement. Jonsell et al. (2006) present a statistical argument against this most popular scenario. The probability of finding an AGB star and a type-II supernova polluting a main-sequence star should be lower than finding a type-II supernova alone polluting a main-sequence star. Therefore, the probability of finding CEMP-rs stars should be lower than finding rII stars (which were only polluted by a type-II supernova). According to Jonsell et al. (2006), this is not supported by the available statistics, since CEMP-rs stars are more numerous than rII stars. According to the same argument, we should find fewer CEMP-rs stars than CEMP-s stars. However, in Fig. 1, there are instead almost equal numbers. In our picture, the statistics has a straightforward explanation in terms of the IMF. If we consider that companions to CEMP-s stars had an initial mass in the range $1-3 M_{\odot}$, whereas CEMP-rs stars have 3-8 $M_{\odot}$ companions, the resulting CEMPrs to CEMP-s frequency ratio is 0.62 adopting a Miller \& Scalo (1979) IMF and 0.95 adopting a Lucatello et al. (2005a) IMF. With the same IMFs, we would find frequency ratios of 0.68 and 1.01 , when taking $2.4 M_{\odot}$ instead of $3 M_{\odot}$ as the threshold between the two classes. Although this estimate requires more stringent constraints on the masses from theoretical models and more accurate observed statistics, it appears natural that CEMPrs are approximately as numerous as CEMP-s stars, and still more numerous than rII stars.

\section{CEMP-no stars}

\subsection{Absence of neutron-capture signature}

In the case of CEMP-s and CEMP-rs stars, a strong argument in favour of mass transfer from an AGB companion is the enhancement in $s$-process elements. For those stars, the analysis was simplified by assuming that the AGB contribution to the observed heavy-element abundances overwhelms the primordial abundances in the accreting star. Unfortunately, for CEMP-no 

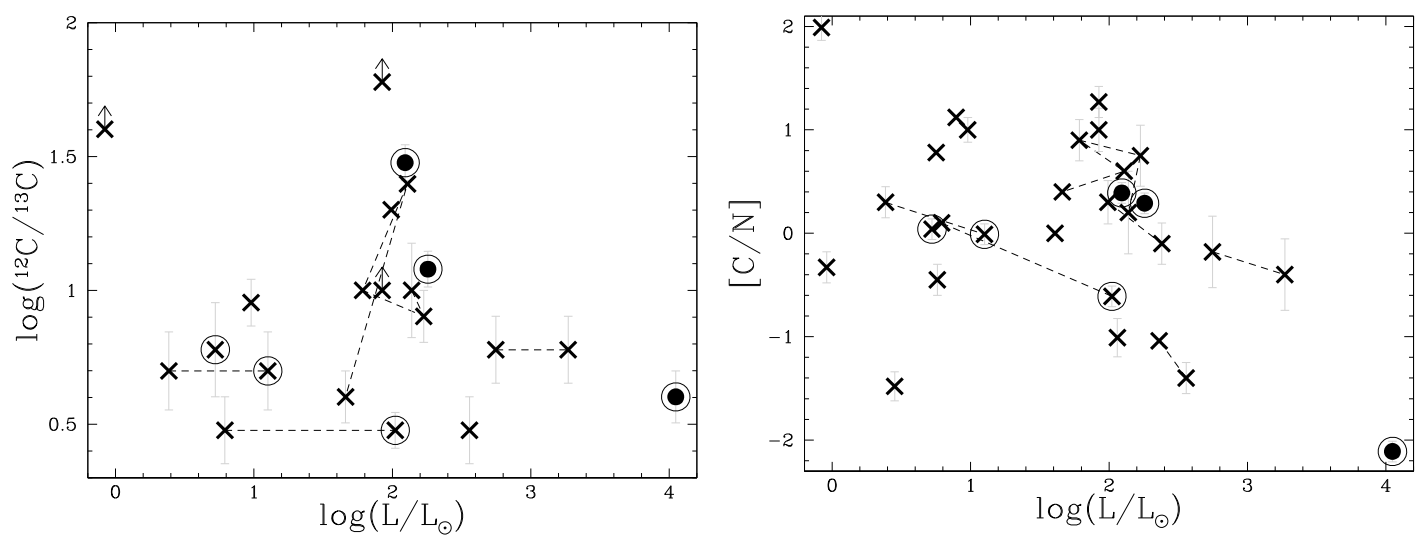

Fig. 19. $\log \left({ }^{12} \mathrm{C} /{ }^{13} \mathrm{C}\right)$ and $[\mathrm{C} / \mathrm{N}]$ as a function of luminosity in CEMP-no stars. Some main sequence CEMP-no stars with enhanced $\mathrm{N}$ and and low ${ }^{12} \mathrm{C} /{ }^{13} \mathrm{C}$ ratios exist.

stars, this assumption does not hold true because CEMP-no stars have, by definition, low abundances of the neutron-capture elements. Because the neutron-capture enrichment for field stars is also quite variable, we cannot reliably separate contributions of the AGB star from the pristine (unknown) abundances (Fig. 4). The $\mathrm{C}$ and $\mathrm{N}$ enhancements in CEMP-no stars resemble those in CEMP-s and CEMP-rs stars. However, models for both lowmetallicity massive stars and low-metallicity AGB stars predict $\mathrm{C}$ and $\mathrm{N}$ enhancements (e.g., Hirschi 2007; Siess et al. 2004). As remarked in Sect. 1, there is a lack of radial-velocity measurements to constrain the binary rate of CEMP-no stars.

Ryan et al. (2005) observed that all CEMP-no stars in their compilation were post-main-sequence stars, leading these authors to suggest that CEMP-no stars have undergone first dredgeup and have processed some pristine $\mathrm{C}$ into $\mathrm{N}$ themselves. In their scenario, CEMP-no stars were born out of gas with high $\mathrm{C}$ content from pollution by (possibly) low-energy supernovae or winds from massive stars, $\mathrm{C}$ that was then processed to produce the high $\mathrm{N}$ and low ${ }^{12} \mathrm{C} /{ }^{13} \mathrm{C}$ in the CEMP-no stars in their sample. However, as already noted by Aoki et al. (2007), some main sequence CEMP-no stars exist with similar $\mathrm{N}$ enhancements and similar ${ }^{12} \mathrm{C} /{ }^{13} \mathrm{C}$ ratios as CEMP-no giants (Fig. 19), thus ruling out the hypothesis of Ryan et al. (2005). Therefore, if supernovae and/or massive winds are responsible for the $\mathrm{C}, \mathrm{N}$, and isotopic ratios, the yields from these objects must already bear the signature of $\mathrm{CN}$-processed material at the time it leaves the massive star. This may be possible with rotating stars (e.g., Meynet \& Maeder 2002; Hirschi 2007) so we cannot exclude SN/massive stars based on this argument alone, although AGB stars can also easily produce $\mathrm{CN}$-processed material.

\subsection{CEMP-no stars: the extremely metal-poor counterparts of CEMP s-process-rich (CEMP-s+CEMP-rs) stars}

Without clear diagnostics such as mass (from an orbital solution), or $s$ - or $r$-process abundance patterns, the nature of the companion that polluted the CEMP-no star is very difficult to assess on a star by star basis. However, because of our holistic approach, we may invoke several arguments collectively pointing towards the scenario of a mass transfer from a former AGB companion:

+ It is remarkable that CEMP-low-s stars share many properties with CEMP-no stars: not only $[\mathrm{Ba} / \mathrm{Fe}]<1$ (their defining property), but also mild $\mathrm{C}$-enrichments and similar $[\mathrm{C}+\mathrm{N} / \mathrm{H}]$ and $[\mathrm{C}+\mathrm{N}+\mathrm{O} / \mathrm{H}]$ ratios (Aoki et al. 2002c, Figs. 20 and 21).

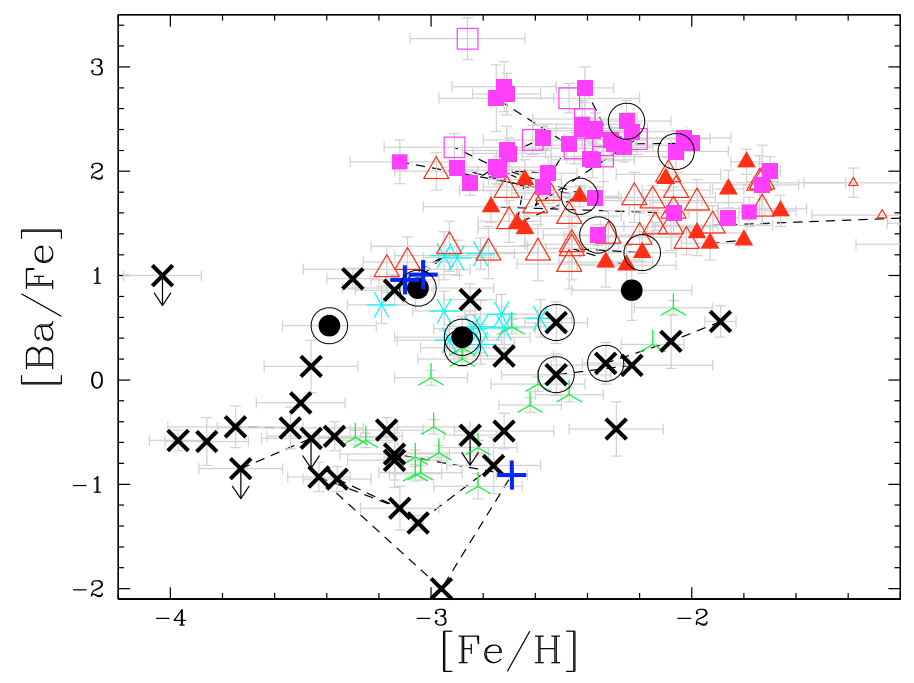

Fig. 20. $[\mathrm{Ba} / \mathrm{Fe}]$ as a function of metallicity for all CEMP stars (see Fig. 2 for a description of the symbols).

It was established in Sect. 4 that CEMP-low-s stars have $\mathrm{Ba}$ and Eu abundances compatible with the $s$-process, and hence, owe their peculiarities to AGB mass transfer (excluding the CEMP-low-s star CS 30322-023, which is an intrinsic AGB star). We can also add to these three CEMP-lows stars (black dots in Figs. 20 and 21) the CEMP-no star CS 22956-28 (a blue straggler), which has been shown to be a binary with evidence of mass transfer from a former AGB companion (Sneden et al. 2003b). There are thus hints of an AGB mass transfer in at least 4 CEMP-no or CEMP-lows stars. Given the many similarities between CEMP-no and CEMP-low-s stars (the former could even become CEMPlow-s stars when their Eu abundance becomes available), it may thus be suspected that AGB mass transfer also plays a role in many CEMP-no stars.

+ There is a clear continuity in the abundance trends for CEMP-s, CEMP-rs, and CEMP-no stars as a function of metallicity, especially for O (Fig. 18), C+N (Fig. 21), and $\mathrm{Mg}$ (Fig. 22). Moreover, Fig. 17 reveals that CEMP-no stars have ${ }^{12} \mathrm{C} /{ }^{13} \mathrm{C}$ and $\mathrm{C} / \mathrm{N}$ ratios close to CEMP-s stars and CEMP-rs stars. The CEMP-no stars are apparently divided into two subcategories, the $\mathrm{O}$ - and $\mathrm{Mg}$-enhanced and the $\mathrm{O}$ and Mg-normal, as already noted by Aoki et al. (2002a). By looking at Figs. 18 and 22, these two subcategories may 
T. Masseron et al.: A holistic approach to carbon-enhanced metal-poor stars
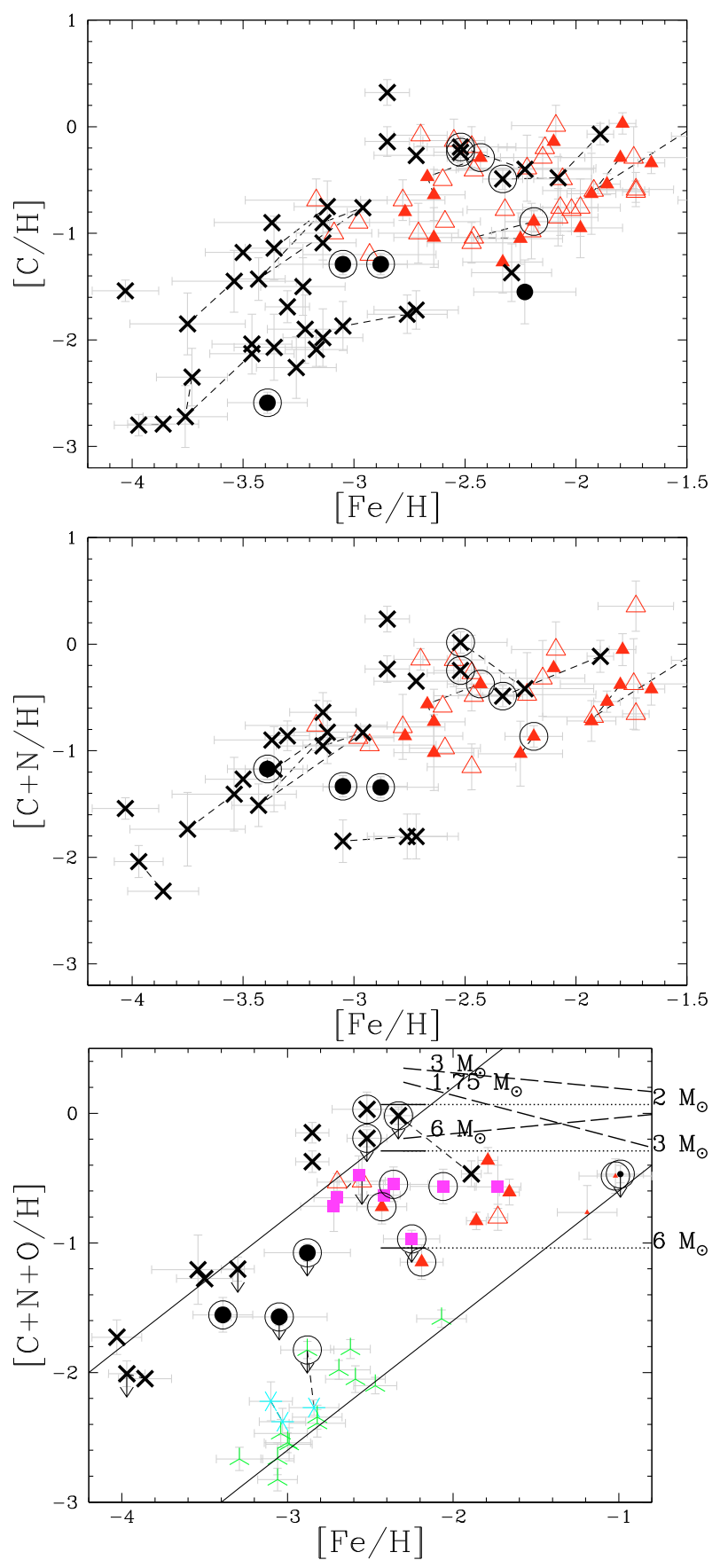

Fig. 21. Top and middle panels: $[\mathrm{C}+\mathrm{N} / \mathrm{H}]$ and $[\mathrm{C} / \mathrm{H}]$ vs metallicity in CEMP-no and CEMP-s stars (see Fig. 2 for a description of symbols). $\mathrm{C}$ and $\mathrm{C}+\mathrm{N}$ abundances decline towards low metallicities in CEMPno stars. Bottom panel: $\mathrm{C}+\mathrm{N}+\mathrm{O}$ vs metallicity in CEMP stars. The long-dashed line corresponds to predictions from Karakas \& Lattanzio (2007), the dotted line to predictions from Herwig (2004) for an AGB star with $[\mathrm{Fe} / \mathrm{H}]=-2.3$. The thin solid lines correspond to $[\mathrm{C}+\mathrm{N}+\mathrm{O} / \mathrm{Fe}]=0.4$ and 2.2. $\mathrm{C}+\mathrm{N}+\mathrm{O}$ stays constant in CEMP-s and CEMP-rs stars (red triangles and magenta squares); on the contrary, $\mathrm{C}+\mathrm{N}+\mathrm{O}$ is proportional to metallicity in CEMP-no stars (black crosses).

naturally be related to CEMP-rs stars and CEMP-s stars, respectively. In the current discussion, we do not consider the 2 most metal-poor stars known to date (HE 0107-5240 and HE 1327-2326), both being C-rich and Ba-poor. For CEMP-s and CEMP-rs stars, we previously demonstrated that metallicity plays an important role in the outcome of nucleosynthesis, and we therefore consider that the metallicity of these

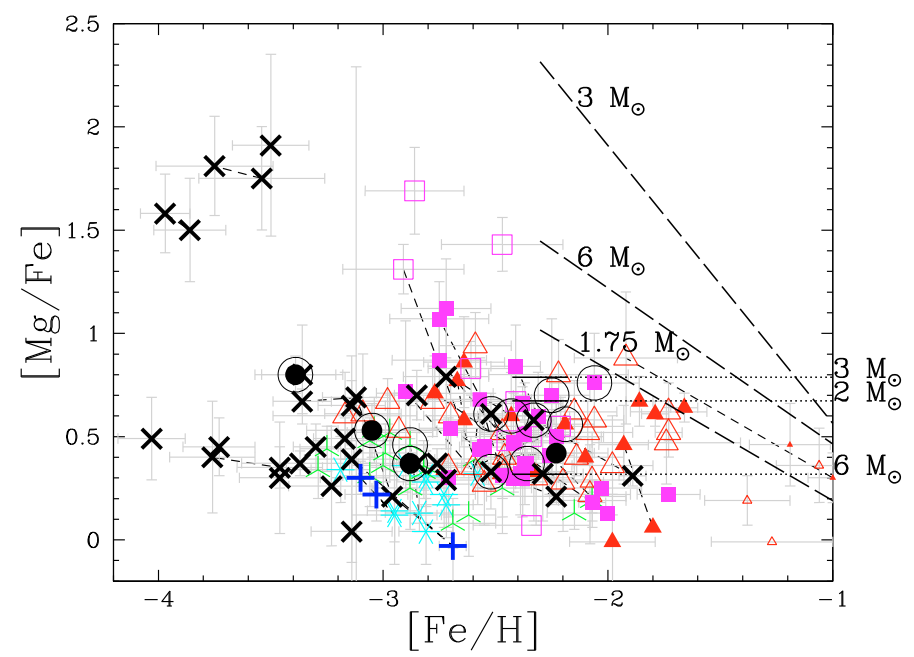

Fig. 22. $\mathrm{Mg}$ enhancement as a function of metallicity for metal-poor stars (see Fig. 2 for a description of symbols). At extremely low metallicities, the Mg enhancement seems to divide CEMP-no stars into 2 categories, some being very $\mathrm{Mg}$-enhanced as some CEMP-rs are, while most of the others do not show any $\mathrm{Mg}$ enhancement.

two record-holders is too different from the bulk of the sample to be safely included in the comparison.

+ As already noticed by Aoki et al. (2007) and shown in Fig. 23, CEMP-no stars are more numerous at low metallicity than CEMP-s and CEMP-rs stars. It is even more puzzling that neither CEMP-rs stars nor CEMP-s stars have been discovered below $[\mathrm{Fe} / \mathrm{H}]<-3.2$. It is unlikely that binaries involving a low- or intermediate-mass AGB star did not form at these very low metallicities. Since the lines from neutron-capture elements have a negligible impact on the stellar colors, it is very unlikely that the absence of CEMP-s stars at low metallicities results from a selection effect acting against their detection when using broad-band colours as in the HE or HK surveys. Thus, CEMP-no stars seem to be good candidates for being the more metal-poor counterparts of CEMP-s and/or CEMP-rs stars.

Although several observational facts seem to indicate that most of the CEMP-no stars had an AGB companion, it remains impossible to determine the origin of CEMP-no stars on an individual basis. Indeed, it is still possible that the abundances of some of the CEMP-no stars reflect the yields of the early massive stars with high $\mathrm{C}$ and $\mathrm{N}$ enhancements.

Beyond the debate relating or not relating CEMP-no stars to AGB companions, the observed lack of CEMP-s stars of metallicities $[\mathrm{Fe} / \mathrm{H}] \lesssim-3.0$ might highlight a fundamental difference in the properties of extremely-low AGB stars compared to more metal-rich ones. Based on the natural assumption that any lowand intermediate-mass star undergoes an AGB phase, irrespective of its metallicity, the changes observed in the abundances of CEMP stars between the metal-poor regime $(-3.0 \lesssim[\mathrm{Fe} / \mathrm{H}]$ $\lesssim-1.5)$ and the extremely metal-poor regime $([\mathrm{Fe} / \mathrm{H}] \lesssim-3.0)$ must reflect similar changes in the properties of the $s$-process nucleosynthesis occurring in AGB stars in these two metallicity regimes. We now review possible causes of these differences.

Is the $s$-process pushed toward the third peak at very low metallicities? When ${ }^{13} \mathrm{C}(\alpha, \mathrm{n}){ }^{16} \mathrm{O}$ is the neutron source in AGB stars, models predict that the efficiency of the $s$-process, expressed in terms of the number of neutrons captured per neutron seed nuclei, increases with decreasing metallicity (Clayton 1988; Goriely \& Siess 2001; Busso et al. 2001). The $s$-process 


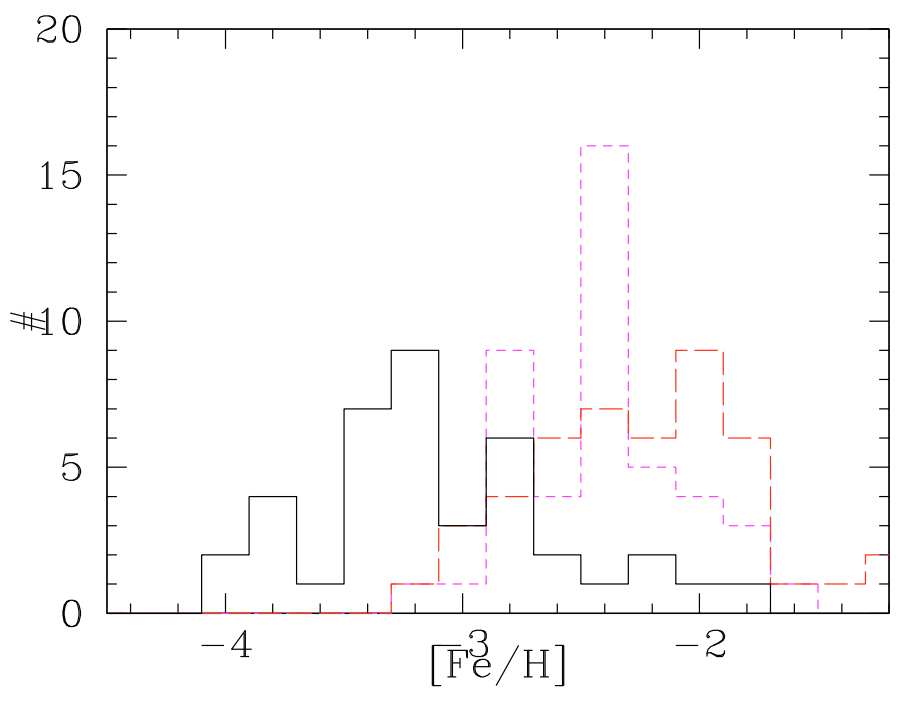

Fig. 23. Number of stars in the different CEMP subclasses in our sample as a function of metallicity: the black solid line represents CEMP-no stars, the red long-dashed line CEMP-s stars, and the short-dashed magenta line CEMP-rs stars. CEMP-no stars in general show up at lower metallicities than the other CEMP stars.

enrichment may not be obvious to detect then, because it may be restricted to a large overabundance involving the sole $\mathrm{Pb}$. The $\mathrm{Pb}$ measurements available in CEMP-low-s stars and the few upper limits for CEMP-no stars do not infer strong overabundances, so that this possibility is excluded out (Fig. 24). We note that, because $\mathrm{Pb}$ has a condensation temperature similar to that of $\mathrm{Na}$ and $\mathrm{S}$ (Lodders 2003), the lack of $\mathrm{Pb}$ enhancement also confirms the statement of Venn \& Lambert (2008) that peculiar abundances in CEMP-no stars with $[\mathrm{Fe} / \mathrm{H}]>-4.0$ cannot be explained by re-accretion of dust-to-gas segregated material (as in $\lambda$ Boo stars).

If CEMP-no stars are the metal-poor counterparts of CEMP stars with $s$-process enhancements, what are the few metalrich CEMP-no stars (with $[\mathrm{Fe} /]>-2.5$ on Fig. 23)? It is useful to investigate in more detail the properties of the relatively high-metallicity $([\mathrm{Fe} / \mathrm{H}]>-2.5)$ CEMP-no stars, such as HKII 17435-00532, HE 1330-0354, CS 22956-028, CS 22945017, and HE 1410+0213, and compare them to those of the CEMP-s stars of the same metallicity. The abundance pattern of the first star is compatible with a strong dilution of the accreted material in a $r$-process-rich gas (see Sect. 7.2). The second one also shows a mild enrichment in both $\mathrm{C}$ and $\mathrm{Ba}$. Therefore, the dilution scenario is also plausible for this star. Nevertheless, the abundance pattern of the last three ones is more puzzling. While they all exhibit a $\mathrm{C}$ abundance comparable to CEMP-s stars, CS 22956-028 is recognized as a blue straggler (Sneden et al. 2003b, Paper II) and HE 1410+0213 is extremely O-enhanced in the same manner as in more metal-poor CEMP-no stars. We note that CS 22956-028 being a blue straggler is consistent with the large amount of material accreted having modified its evolution, similarly to what is observed in CEMP-rs stars (Sect. 6.2). Thus, the various abundance patterns of these relatively metalrich CEMP-no stars may come from various progenitors: accretion of matter from either an intermediate-mass AGB star (with oxygen coming from ${ }^{12} \mathrm{C}(\alpha, \gamma){ }^{16} \mathrm{O}$ reaction operating in warm thermal pulses), or even from a massive star having exploded as a type II supernova. Therefore, their connection with more metal-poor CEMP-no stars is not straightforward, and a more detailed study of these objects needs to be completed.

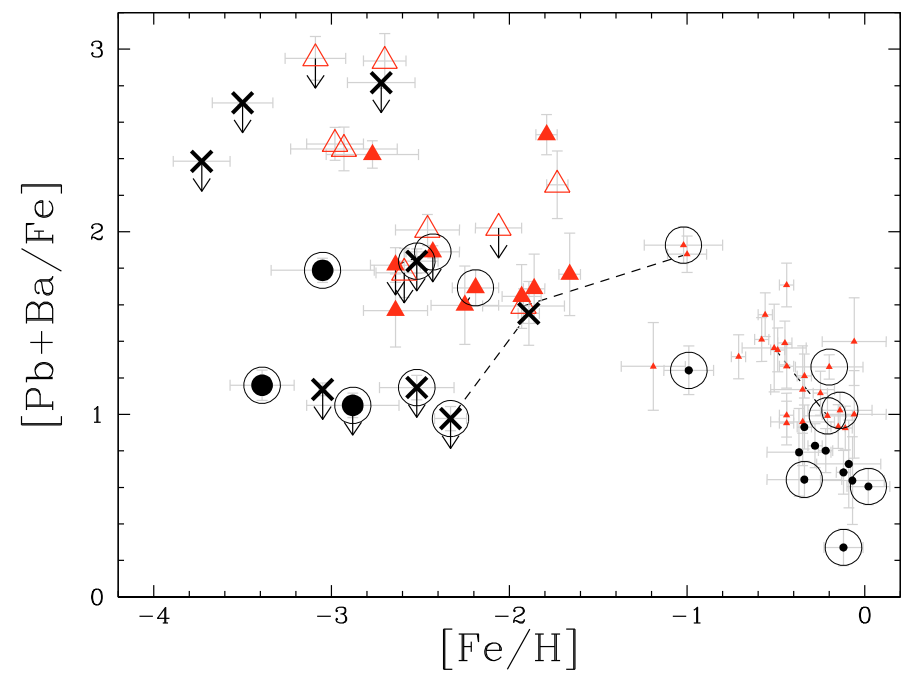

Fig. 24. $\mathrm{Pb}+\mathrm{Ba}$ enhancement as a function of metallicity in Ba stars, CEMP-s, and CEMP-no stars (see Fig. 2 for a description of symbols). $[\mathrm{Pb}+\mathrm{Ba} / \mathrm{Fe}]$ is lower in CEMP-no stars than in CEMP-s stars.

It is likely that the complex interplay between mass and metallicity has a decisive impact on the AGB nucleosynthesis at extremely low metallicity. Besides increasing the temperature at which the nucleosynthesis operates, the low metallicity regime might also enhance the effects of rotation on the abundances (Herwig et al. 2003; Siess et al. 2004; Decressin \& Charbonnel 2006) or might induce the occurrence of the hydrogen injection flash during the thermal pulse (aka He-FDDM, Fujimoto et al. 2000). Rotation is predicted to enhance $\mathrm{N}$ in agreement with that observed for most CEMP-no stars and contrasting with CEMP-s and CEMP-rs stars (Ryan et al. 2005, and Fig. 17). When rotation increases the $\mathrm{N}$ production, it inhibits the $s$-process but is not predicted to enhance $\mathrm{O}$ in AGB stars (as observed for CEMPno stars in Fig. 18). He-FDDM has a drastic impact on the AGB structure, and is consequently expected to modify the AGB nucleosynthesis. Although the metallicity threshold below which this latter mechanism operates is still debated, this effect is predicted for all AGB models at low metallicity (Fujimoto et al. 2000; Siess et al. 2002; Herwig 2005; Campbell \& Lattanzio 2008), and may explain the increase in CEMP-no stars at extremely low metallicity. More extensive theoretical studies of AGB stars with metallicities in the range $-5.0<[\mathrm{Fe} / \mathrm{H}]<-3.0$ are needed to evaluate the importance of these effects in CEMPno stars.

Finally, there is one striking result that is difficult to reconcile with the binary scenario. Figure 21 shows that $\mathrm{C}$ declines in proportion to metallicity in CEMP-no stars, as do $[\mathrm{C}+\mathrm{N} / \mathrm{H}]$ and $[\mathrm{C}+\mathrm{N}+\mathrm{O} / \mathrm{H}]$. This is very puzzling because the $\mathrm{C}$ yields from both AGB and massive stars yields is of a primary nature (i.e., independent of metallicity), thus its observed abundance should similarly be independent of metallicity. When considering the effect of rotation in massive stars, the expected $\mathrm{C}$ and $\mathrm{O}$ abundances should even follow the opposite trend (Hirschi 2007).

\section{Summary}

The analysis of abundances in CEMP stars (summarised in Table 5) leads to the following conclusions:

- Ba stars are the metal-rich counterparts of CEMP-s stars.

- We demonstrate that CEMP-low-s stars are very likely to be the result of mass transfer from an AGB companion because 
Table 5. Summary of the properties of CEMP stars.

\begin{tabular}{|c|c|c|c|c|c|}
\hline & CEMP-no & CEMP-low-s & CEMP-S & CEMP-rs & Comment \\
\hline$\overline{\mathrm{C}}$ & medium, $\nearrow$ with Fe & medium & high & high & from AGB or low-Fe SNII \\
\hline${ }^{12} \mathrm{C} /{ }^{13} \mathrm{C}$ & low to high & low to high & low to high & low to high & signature of CN cycling \\
\hline $\mathrm{N}$ & high & high & high & high & not from $\mathrm{HBB}$, from cool bottom processing, rotation...? \\
\hline $\mathrm{O}$ & high in most & std & std & high in some & SNII or high temperature pulses ? \\
\hline $\mathrm{Mg}$ & high & high in some & std & high in some & ${ }^{22} \mathrm{Ne}(\alpha, n){ }^{25} \mathrm{Mg}$ or ${ }^{20} \mathrm{Ne}(\alpha, \gamma){ }^{24} \mathrm{Mg} ?$ \\
\hline $\mathrm{Ba}$ & low & low & high & very high & correlated with $\mathrm{C}$ for CEMP-s and low-s \\
\hline $\mathrm{La}$ & low & low & high & very high & from ${ }^{13} \mathrm{C}(\alpha, n){ }^{16} \mathrm{O}$ in CEMP-s and ${ }^{22} \mathrm{Ne}(\alpha, n){ }^{25} \mathrm{Mg}$ in CEMP-rs \\
\hline $\mathrm{Ce}$ & low & low & high & high and cst & ,", \\
\hline $\mathrm{Eu}$ & not measurable & low & medium & high & ", \\
\hline $\mathrm{Pb}$ & not measured & low & medium & high & ,", \\
\hline
\end{tabular}

of their $\mathrm{C}$ and $s$-process signatures. Although their light element-abundance pattern is identical to that of CEMP-no stars, it is not clear yet whether or not all CEMP-no stars are CEMP-low-s stars.

- The neutron-capture element ratios in CEMP-s stars are consistent with low-mass AGB models where the ${ }^{13} \mathrm{C}$ neutron source has operated.

- The observed relation between the $\mathrm{Ba} / \mathrm{C}$ ratio and metallicity in CEMP-s stars provides strong constraints on the mechanism responsible for the formation of ${ }^{13} \mathrm{C}$ in the He-rich shell during the interpulse.

- The low dispersion in $\mathrm{Ce} / \mathrm{Fe}$ as a function of luminosity in CEMP-rs stars suggests that a large amount of material (with a fixed $\mathrm{Ce}$ abundance) has been dumped onto the CEMP-rs star envelope, thus erasing all variations caused by the dispersion in all the initial abundances by the so-called thermohaline mixing.

- The correlation between [vhs/hs] and $[\mathrm{N} / \mathrm{H}]$ ratios in CEMPrs stars as well as the $[\mathrm{Mg} / \mathrm{Fe}]$ in some of them suggest the operation of the ${ }^{22} \mathrm{Ne}$ neutron source.

- All CEMP stars have low ${ }^{12} \mathrm{C} /{ }^{13} \mathrm{C}$ ratios, confirming that $\mathrm{CN}$ processing occurring in low-metallicity AGB stars is responsible for the observed $\mathrm{N}$, but the high $[\mathrm{C} / \mathrm{N}]$ ratios suggest that the conditions offered by $\mathrm{HBB}$ in the convective envelope are inadequate to produce the observed N. In contrast, these ratios points toward characteristics compatible with cool bottom processing.

- CEMP-rs stars exhibit O enhancement compared to CEMP$\mathrm{S}$ stars; this implies that temperatures were higher in the He-burning shell of CEMP-rs companion than in the CEMP-s companion, in agreement with the operation of ${ }^{22} \mathrm{Ne}(\alpha, \mathrm{n}){ }^{25} \mathrm{Mg}$.

- We highlight the possible relation between CEMP-no stars and AGB stars. Hence, most CEMP-no stars are likely to be the metal-poor counterparts of CEMP-s and CEMP-rs stars.

- The decline in $\mathrm{C}$ and the $s$-process in CEMP-no stars points infers lower $\mathrm{C}$ production and inefficient $s$-process in lowmetallicity AGB stars. These two observation do not have any explanation in current AGB models.

Nevertheless, one may attempt to use these constraints to sort CEMP subclasses according to the mass of the progenitor. The hotter conditions achieved in the He-burning shell of the CEMPrs stars companions than in the CEMP-s stars companions suggest that CEMP-rs stars had more massive AGB companions than CEMP-s stars. Furthermore, there is now a consensus for attributing the peculiar abundances of CEMP-s stars to lowmass AGB stars. Since the intermediate-mass counterparts of CEMP-s stars have not yet been identified (Johnson et al. 2007), CEMP-rs stars remain the best candidates. This interpretation is also in agreement with the high frequency of CEMP-rs stars
(Jonsell et al. 2006). Concerning CEMP-no stars, they are likely to encompass both intermediate- and low-mass AGB stars at extremely low metallicity $([\mathrm{Fe} / \mathrm{H}] \lesssim-3.0)$. Indeed, the low metallicity regime seems to have drastic effect on nucleosynthesis.

However, the conditions for making $\mathrm{N}$ in the H-rich layer of the AGB companion to the CEMP stars have not yet been well identified, since the $[\mathrm{C} / \mathrm{N}]$ ratios are very similar in all categories of CEMP stars. Furthermore, it is expected that the more massive the AGB star, the more the synthesized material is diluted in the AGB envelope, and thus the lower are the yields. This is in contradiction with the overabundances of neutron-capture elements being larger in CEMP-rs stars than in CEMP-s stars (Fig. 23). Nevertheless, we also emphasize that the ${ }^{22} \mathrm{Ne}(\alpha, \mathrm{n})^{25} \mathrm{Mg}$ process can be very efficient in producing $s$-process elements. AGB models are also affected by uncertainties, in particular the impact of HBB, the mechanism of CBP, the parametrization of $3^{\text {rd }}$ dredge-ups, the number of thermal pulses, the occurrence of the hydrogen injection flash (Fujimoto et al. 2000), the possible effects of both rotation (Siess et al. 2004) and thermohaline mixing (Cantiello \& Langer 2008), and finally, the impact of the uncertainty on the ${ }^{22} \mathrm{Ne}(\alpha, \mathrm{n})^{25} \mathrm{Mg}$ reaction rate. Therefore, it is not at all straightforward to deduce the mass of the former AGB companion of CEMP stars from the analysis of their abundances.

\subsection{Open questions}

- What is the mechanism for $\mathrm{N}$ production in AGB stars? Is $\mathrm{HBB}$ really effective at low metallicity?

- Since N/C and O/Fe seem to increase with decreasing metallicity, should we not search for NEMP or OEMP stars? However, only a couple of N-rich metal-poor stars have been found so far (Johnson et al. 2007).

- Where are the CEMP-s and CEMP-rs stars at extremely low metallicity?

- Do metal-rich counterparts to CEMP-rs stars exist? The $\mathrm{N}$-rich and Rb-rich stars from García-Hernández et al. (2006) might be possible candidates.

- Are all CEMP-no stars the results of AGB mass transfer?

- How low in metallicity does the $\mathrm{C}$ and $s$-process decline go? Do HE 1327-2326 and HE 0107-5240 fit into this scenario ?

- What is the impact of the observed $\mathrm{C}$ decrease at very low metallicity on CEMP statistics? Do the strongest enhancements of elements in these stars (in particular $\mathrm{N}, \mathrm{O}, \mathrm{Mg}, s-$ process) have any implications for the chemical evolution of the Galaxy?

Acknowledgements. T.M. deeply thanks P. Denissenkov, M. Pinsonneault and M. Lugaro for fruitful discussions. T.M. gratefully acknowledge support from ESO studentship program and from CNRS/INSU. T.M. and J.A.J. also acknowledge support from AST-0707948. 
A\&A 509, A93 (2010)

\section{References}

Abia, C., Busso, M., Gallino, R., et al. 2001, ApJ, 559, 1117

Allen, D. M., \& Barbuy, B. 2006a, A\&A, 454, 895

Allen, D. M., \& Barbuy, B. 2006b, A\&A, 454, 917

Anders, E., \& Grevesse, N. 1989, Geochim. Cosmochim. Acta, 53, 197

Aoki, W., Ryan, S. G., Norris, J. E., et al. 2001, ApJ, 561, 346

Aoki, W., Norris, J. E., Ryan, S. G., Beers, T. C., \& Ando, H. 2002a, ApJ, 576, L141

Aoki, W., Norris, J. E., Ryan, S. G., Beers, T. C., \& Ando, H. 2002b, PASJ, 54, 933

Aoki, W., Norris, J. E., Ryan, S. G., Beers, T. C., \& Ando, H. 2002c, ApJ, 567, 1166

Aoki, W., Ryan, S. G., Norris, J. E., et al. 2002d, ApJ, 580, 1149

Aoki, W., Norris, J. E., Ryan, S. G., et al. 2004, ApJ, 608, 971

Aoki, W., Bisterzo, S., Gallino, R., et al. 2006, ApJ, 650, L127

Aoki, W., Beers, T. C., Christlieb, N., et al. 2007, ApJ, 655, 492

Aoki, W., Beers, T. C., Sivarani, T., et al. 2008, ApJ, 678, 1351

Asplund, M. 2005, ARA\&A, 43, 481

Barbuy, B., Jorissen, A., Rossi, S. C. F., et al. 1992, A\&A, 262, 216

Barbuy, B., Cayrel, R., Spite, M., et al. 1997, A\&A, 317, L63

Barbuy, B., Spite, M., Spite, F., et al. 2005, A\&A, 429, 1031

Barklem, P. S., Christlieb, N., Beers, T. C., et al. 2005, A\&A, 439, 129

Beers, T. C., \& Christlieb, N. 2005, ARA\&A, 43, 531

Beers, T. C., Preston, G. W., \& Shectman, S. A. 1992, AJ, 103, 1987

Bisterzo, S., \& Gallino, R. 2008, in Evolution and Nucleosynthesis in AGB Stars,

ed. R. Guandalini, S. Palmerini, \& M. Busso, AIP Conf. Ser., 1001, 131

Bisterzo, S., Gallino, R., Straniero, O., et al. 2006, MNRAS, 77, 985

Bonifacio, P., Molaro, P., Beers, T. C., et al. 1998, A\&A, 332, 672

Boothroyd, A. I., \& Sackmann, I.-J. 1999, ApJ, 510, 232

Busso, M., Gallino, R., Lambert, D. L., Travaglio, C., \& Smith, V. V. 2001, ApJ, 557,802

Campbell, S. W., \& Lattanzio, J. C. 2008, A\&A, 490, 769

Cantiello, M., \& Langer, N. 2008, in IAU Symp., 252, ed. L. Deng, \& K. L. Chan, 103

Cayrel, R., Depagne, E., Spite, M., et al. 2004, A\&A, 416, 1117

Christlieb, N., Green, P. J., Wisotzki, L., et al. 2001, A\&A, 375, 366

Christlieb, N., Bessell, M. S., Beers, T. C., et al. 2002, Nature, 419, 904

Christlieb, N., Gustafsson, B., Korn, A. J., et al. 2004, ApJ, 603, 708

Clayton, D. D. 1988, MNRAS, 234, 1

Cohen, J. G., Christlieb, N., Qian, Y.-Z., et al. 2003, ApJ, 588, 1082

Cohen, J. G., Christlieb, N., McWilliam, A., et al. 2004, ApJ, 612, 1107

Cohen, J. G., McWilliam, A., Shectman, S., et al. 2006, AJ, 132, 137

Cohen, J. G., Christlieb, N., McWilliam, A., et al. 2008, ApJ, 672, 320

Cristallo, S., Straniero, O., Gallino, R., et al. 2009, ApJ, 696, 797

Decressin, T., \& Charbonnel, C. 2006, in International Symposium on Nuclear Astrophysics - Nuclei in the Cosmos, PoS(NIC-IX)086

Denissenkov, P. A., \& Pinsonneault, M. 2008, ApJ, 679, 1541

Depagne, E., Hill, V., Spite, M., et al. 2002, A\&A, 390, 187

Deroo, P., Reyniers, M., van Winckel, H., Goriely, S., \& Siess, L. 2005, A\&A, 438, 987

François, P., Depagne, E., Hill, V., et al. 2007, A\&A, 476, 935

Frebel, A., Aoki, W., Christlieb, N., et al. 2005, Nature, 434, 871

Frebel, A., Christlieb, N., Norris, J. E., Aoki, W., \& Asplund, M. 2006, ApJ, 638, L17

Frebel, A., Norris, J. E., Aoki, W., et al. 2007, ApJ, 658, 534

Fujimoto, M. Y., Ikeda, Y., \& Iben, I. J. 2000, ApJ, 529, L25

Gallino, R., Arlandini, C., Busso, M., et al. 1998, ApJ, 497, 388

García-Hernández, D. A., García-Lario, P., Plez, B., et al. 2006, Science, 314, 1751

García Pérez, A. E., Asplund, M., Primas, F., Nissen, P. E., \& Gustafsson, B. 2006, A\&A, 451, 621

Gilroy, K. K. 1989, ApJ, 347, 835

Goriely, S. 1999, A\&A, 342, 881

Goriely, S., \& Mowlavi, N. 2000, A\&A, 362, 599

Goriely, S., \& Siess, L. 2001, A\&A, 378, L25

Goriely, S., \& Siess, L. 2004, A\&A, 421, L25

Goriely, S., \& Siess, L. 2005, in From Lithium to Uranium: Elemental Tracers of Early Cosmic Evolution, ed. V. Hill, P. François, \& F. Primas, IAU Symp., 451

Goswami, A., Aoki, W., Beers, T. C., et al. 2006, MNRAS, 372, 343

Grevesse, N., \& Sauval, A. J. 1998, Space Sci. Rev., 85, 161

Grevesse, N., Noels, A., \& Sauval, A. J. 1996, in Cosmic Abundances, ed. S. S.

Holt, \& G. Sonneborn, ASP Conf. Ser., 99, 117

Grevesse, N., Sauval, A., \& Murdin, P. 2000, Solar Abundances
Heger, A., \& Woosley, S. E. 2002, ApJ, 567, 532

Herwig, F. 2004, ApJS, 155, 651

Herwig, F. 2005, ARA\&A, 43, 435

Herwig, F., Langer, N., \& Lugaro, M. 2003, ApJ, 593, 1056

Hill, V., Barbuy, B., Spite, M., et al. 2000, A\&A, 353, 557

Hill, V., Plez, B., Cayrel, R., et al. 2002, A\&A, 387, 560

Hirschi, R. 2007, A\&A, 461, 571

Honda, S., Aoki, W., Ishimaru, Y., Wanajo, S., \& Ryan, S. G. 2006, ApJ, 643, 1180

Honda, S., Aoki, W., Kajino, T., et al. 2004, ApJ, 607, 474

Ivans, I. I., Sneden, C., Gallino, R., Cowan, J. J., \& Preston, G. W. 2005, ApJ, 627, L145

Johnson, J. A., \& Bolte, M. 2002, ApJ, 579, L87

Johnson, J. A., \& Bolte, M. 2004, ApJ, 605, 462

Johnson, J. A., Herwig, F., Beers, T. C., et al. 2007, ApJ, 658, 1203

Jonsell, K., Barklem, P. S., Gustafsson, B., et al. 2006, A\&A, 451, 651

Jorissen, A., \& Van Eck, S. 2000, in The Carbon Star Phenomenon, ed. R. F.

Wing, IAU Symp., 177, 259

Justham, S., Wolf, C., Podsiadlowski, P., et al. 2009, A\&A, 493, 1081

Karakas, A., \& Lattanzio, J. C. 2007, Publications of the Astronomical Society of Australia, 24, 103

Karakas, A. I., \& Lattanzio, J. C. 2003, Publications of the Astronomical Society of Australia, 20, 279

Komiya, Y., Suda, T., Minaguchi, H., et al. 2007, ApJ, 658, 367

Limongi, M., \& Chieffi, A. 2003, ApJ, 592, 404

Lodders, K. 2003, ApJ, 591, 1220

Lucatello, S. 2003, Ph.D. Thesis, Università degli studi di Padova, Italy

Lucatello, S., Gratton, R., Cohen, J. G., et al. 2003, AJ, 125, 875

Lucatello, S., Gratton, R. G., Beers, T. C., et al. 2005a, ApJ, 625, 833

Lucatello, S., Tsangarides, S., Beers, T. C., et al. 2005b, ApJ, 625, 825

Lucatello, S., Beers, T. C., Christlieb, N., et al. 2006, ApJ, 652, L37

Masseron, T. 2006, Ph.D. Thesis, Observatoire de Paris, France

Masseron, T., van Eck, S., Famaey, B., et al. 2006, A\&A, 455, 1059

McClure, R. D., \& Woodsworth, A. W. 1990, ApJ, 352, 709

Meynet, G., \& Maeder, A. 2002, A\&A, 381, L25

Miller, G. E., \& Scalo, J. M. 1979, ApJS, 41, 513

Nollett, K. M., Busso, M., \& Wasserburg, G. J. 2003, ApJ, 582, 1036

Nomoto, K., \& Kondo, Y. 1991, ApJ, 367, L19

Norris, J. E., Ryan, S. G., \& Beers, T. C. 1997, ApJ, 489, L169

Norris, J. E., Christlieb, N., Korn, A. J., et al. 2007, ApJ, 670, 774

Pignatari, M., Gallino, R., Meynet, G., et al. 2008, ApJ, 687, L95

Plez, B., \& Cohen, J. G. 2005, A\&A, 434, 1117

Plez, B., Hill, V., Cayrel, R., et al. 2004, A\&A, 428, L9

Plez, B., Masseron, T., Van Eck, S., et al. 2008, in ASP Conf. Ser. 384, ed. G. Van Belle, 326

Preston, G. W., \& Sneden, C. 2000, AJ, 120, 1014

Preston, G. W., \& Sneden, C. 2001, AJ, 122, 1545

Proffitt, C. R., \& Michaud, G. 1989, ApJ, 345, 998

Roederer, I. U., Frebel, A., Shetrone, M. D., et al. 2008, ApJ, 679, 1549

Rossi, S., Beers, T. C., \& Sneden, C. 1999, in The Third Stromlo Symposium:

The Galactic Halo, ASP Conf. Ser. 165, 264

Ryan, S. G., \& Norris, J. E. 1991, AJ, 101, 1835

Ryan, S. G., Aoki, W., Norris, J. E., et al. 2005, ApJ, 635, 349

Siess, L., Livio, M., \& Lattanzio, J. 2002, ApJ, 570, 329

Siess, L., Goriely, S., \& Langer, N. 2004, A\&A, 415, 1089

Sivarani, T., Bonifacio, P., Molaro, P., et al. 2004, A\&A, 413, 1073

Sivarani, T., Beers, T. C., Bonifacio, P., et al. 2006, A\&A, 459, 125

Sneden, C., Cowan, J. J., Lawler, J. E., et al. 2003a, ApJ, 591, 936

Sneden, C., Preston, G. W., \& Cowan, J. J. 2003b, ApJ, 592, 504

Spite, M., Cayrel, R., Plez, B., et al. 2005, A\&A, 430, 655

Spite, M., Cayrel, R., Hill, V., et al. 2006, A\&A, 455, 291

Stancliffe, R. J., Glebbeek, E., Izzard, R. G., et al. 2007, A\&A, 464, L57

Straniero, O., Gallino, R., Busso, M., et al. 1995, ApJ, 440, L85

Suda, T., Aikawa, M., Machida, M. N., Fujimoto, M. Y., \& Iben, I. J. 2004, ApJ, 611,476

Takeda, Y. 2003, A\&A, 402, 343

Tsangarides, S. A. 2005, Ph.D. Thesis, Open University (UK), England

Tumlinson, J. 2007, ApJ, 664, L63

Van Eck, S., Goriely, S., Jorissen, A., et al. 2003, A\&A, 404, 291

VandenBerg, D. A., Bergbusch, P. A., \& Dowler, P. D. 2006, ApJS, 162, 375

Vanhala, H. A. T., \& Cameron, A. G. W. 1998, ApJ, 508, 291

Venn, K. A., \& Lambert, D. L. 2008, ApJ, 677, 572

Woosley, S. E., \& Weaver, T. A. 1995, ApJS, 101, 181

Zacs, L., Nissen, P. E., \& Schuster, W. J. 1998, A\&A, 337, 216

Pages 19 to 28 are available in the electronic edition of the journal at http: //www . aanda . org 
T. Masseron et al.: A holistic approach to carbon-enhanced metal-poor stars

Table 1. Abundances of light elements in carbon-enhanced metal-poor stars from literature. Columns (4), (6), (8), (10), (12), (15) list random errors (when available). Column (3) lists metallicities preferentially from Fe I. Column (5) list C abundances from preferencially $\mathrm{CH}$ determinations. (7) $\mathrm{N}$ abundances preferentially from CN. (9) For oxygen abundances (2) stands for measurements from O I triplet and (3) from OH lines. By default, the oxygen measurement comes from the [OI] line at $6300 \AA$. (11) Non-LTE corrections of O I triplet measurement according to Takeda (2003) (when applicable).

\begin{tabular}{|c|c|c|c|c|c|c|c|c|c|c|c|c|c|c|}
\hline $\begin{array}{l}\text { Object } \\
\text { (1) }\end{array}$ & $\begin{array}{c}T_{\text {eff }} \log g \\
\text { (2) }\end{array}$ & $\begin{array}{c}{[\mathrm{Fe} / \mathrm{H}]} \\
(3)\end{array}$ & $\begin{array}{c}\sigma \\
(4)\end{array}$ & $\begin{array}{c}{[\mathrm{C} / \mathrm{Fe}]} \\
(5)\end{array}$ & $\begin{array}{c}\sigma \\
(6)\end{array}$ & $\begin{array}{c}{ }^{12} \mathrm{C} /{ }^{13} \mathrm{C} \\
\text { (7) }\end{array}$ & $\begin{array}{c}\sigma \\
(8)\end{array}$ & $\begin{array}{c}{[\mathrm{N} / \mathrm{Fe}]} \\
(9)\end{array}$ & $\begin{array}{c}\sigma \\
(10)\end{array}$ & $\begin{array}{c}{[\mathrm{O} / \mathrm{Fe}]} \\
(11)\end{array}$ & $\begin{array}{c}\sigma \\
(12)\end{array}$ & $\begin{array}{c}\text { Non-LTE } \\
\text { (13) }\end{array}$ & $\begin{array}{c}{[\mathrm{Mg} / \mathrm{Fe}]} \\
(14)\end{array}$ & $\begin{array}{c}\sigma \\
(15)\end{array}$ \\
\hline HE 0007-1832 & 65153.8 & -2.72 & 0.19 & 2.45 & & $\ldots$ & $\ldots$ & 1.67 & $\ldots$ & $\ldots$ & $\ldots$ & $\ldots$ & 0.79 & 0.35 \\
\hline HE 0012-1441 & 57303.50 & -2.59 & 0.16 & 1.70 & $\ldots$ & $\ldots$ & $\ldots$ & 0.59 & $\ldots$ & $\ldots$ & $\ldots$ & $\ldots$ & 0.94 & 0.16 \\
\hline HE $0024-2523$ & 66254.3 & -2.70 & 0.12 & 2.62 & 0.10 & 6 & 1 & 2.12 & 0.10 & (2) 0.65 & $\ldots$ & $\mathrm{NaN}$ & 0.60 & 0.06 \\
\hline HE $0107-5240$ & 51002.2 & -5.39 & & 3.85 & 0.15 & $>30$ & $\ldots$ & 2.39 & 0.15 & $\ldots$ & $\ldots$ & $\ldots$ & 0.24 & \\
\hline HE 0131-3953 & 59283.83 & -2.71 & 0.17 & 2.32 & 0.27 & $\ldots$ & $\ldots$ & $\ldots$ & $\ldots$ & $\ldots$ & $\ldots$ & $\ldots$ & 0.30 & 0.24 \\
\hline \multirow[t]{2}{*}{ HE 0143-0441 } & 62403.7 & -2.38 & 0.18 & 2.08 & $\ldots$ & $\ldots$ & $\ldots$ & 1.68 & $\ldots$ & $\ldots$ & $\ldots$ & $\ldots$ & 0.66 & 0.08 \\
\hline & 63704.4 & -2.23 & 0.21 & 1.56 & $\ldots$ & $\ldots$ & $\ldots$ & -0.22 & $\ldots$ & $\ldots$ & $\ldots$ & $\ldots$ & 0.50 & 0.17 \\
\hline HE 0202-2204 & 52801.65 & -1.98 & 0.19 & 1.03 & 0.28 & $\ldots$ & $\ldots$ & $\ldots$ & $\ldots$ & $\ldots$ & $\ldots$ & $\ldots$ & -0.01 & 0.23 \\
\hline HE 0206-1916 & 52002.70 & -2.09 & 0.20 & 2.10 & 0.19 & 15 & 5 & 1.61 & 0.33 & $\ldots$ & $\ldots$ & $\ldots$ & 0.52 & 0.15 \\
\hline HE $0212-0557$ & 50752.15 & -2.34 & 0.26 & 1.84 & & 4 & 1 & 1.04 & $\ldots$ & $\ldots$ & $\ldots$ & $\ldots$ & 0.07 & \\
\hline HE 0231-4016 & 59723.59 & -2.08 & 0.18 & 1.23 & 0.27 & $\ldots$ & $\ldots$ & & . & $\ldots$ & $\ldots$ & $\ldots$ & 0.22 & 0.24 \\
\hline \multirow{2}{*}{ HE $0336+0113$} & 59473.7 & -2.39 & 0.09 & 2.72 & 0.10 & $>60$ & $\ldots$ & 1.72 & 0.20 & $\ldots$ & $\ldots$ & $\ldots$ & 0.32 & 0.20 \\
\hline & 57003.50 & -2.75 & 0.22 & 2.35 & & $>7$ & 1 & 1.55 & $\ldots$ & $\ldots$ & $\ldots$ & $\ldots$ & 1.07 & 0.18 \\
\hline HE 0338-3945 & 61624.09 & -2.42 & 0.10 & 2.13 & 0.15 & $\ldots$ & $\ldots$ & 1.55 & 0.20 & (2) 1.40 & 0.11 & $\ldots$ & 0.30 & 0.09 \\
\hline HE 0400-2030 & 56003.50 & -1.73 & 0.17 & 1.14 & 0.16 & $\ldots$ & $\ldots$ & 2.75 & 0.31 & $\ldots$ & $\ldots$ & $\ldots$ & 0.62 & 0.14 \\
\hline HE 0430-4404 & 62144.27 & -2.07 & 0.17 & 1.31 & 0.26 & $\ldots$ & $\ldots$ & $\ldots$ & $\ldots$ & $\ldots$ & $\ldots$ & $\ldots$ & 0.29 & 0.23 \\
\hline HE 0441-0652 & 49001.40 & -2.47 & 0.20 & 1.38 & 0.19 & $\ldots$ & s ... & 0.89 & 0.24 & $\ldots$ & $\ldots$ & $\ldots$ & 0.35 & 0.15 \\
\hline HE 0507-1653 & 50002.40 & -1.38 & 0.19 & 1.29 & 0.19 & 40 & 15 & 0.80 & 0.32 & $\ldots$ & $\ldots$ & $\ldots$ & 0.19 & 0.12 \\
\hline HE 0557-4840 & 49002.2 & -4.75 & 0.17 & 1.65 & 0.10 & $\ldots$ & $\ldots$ & $\ldots$ & $\ldots$ & $<3.09$ & $\ldots$ & $\ldots$ & 0.25 & 0.07 \\
\hline HE 1001-0243 & 50002.00 & -2.88 & 0.26 & 1.59 & 0.10 & 30 & 5 & 1.20 & 0.10 & $<1.92$ & $\ldots$ & $\ldots$ & 0.37 & 0.12 \\
\hline HE 1005-1439 & 50001.90 & -3.17 & 0.32 & 2.48 & 0.20 & $\ldots$ & $\ldots$ & 1.79 & 0.27 & $\cdots$ & $\ldots$ & $\ldots$ & 0.60 & 0.21 \\
\hline HE 1012-1540 & 56203.4 & -3.50 & 0.17 & 2.32 & $\ldots$ & $\ldots$ & $\ldots$ & 1.20 & $\ldots$ & (3) 2.22 & 0.25 & $\ldots$ & 1.91 & 0.44 \\
\hline HE 1031-0020 & 50802.20 & -2.93 & 0.30 & 1.73 & $\ldots$ & $\ldots$ & $\ldots$ & 2.43 & $\ldots$ & $\ldots$ & $\ldots$ & $\ldots$ & 0.53 & 0.18 \\
\hline HE $1105+0027$ & 61323.45 & -2.42 & 0.19 & 1.87 & 0.28 & $\ldots$ & $\ldots$ & $\ldots$ & $\ldots$ & $\ldots$ & $\ldots$ & $\ldots$ & 0.47 & 0.24 \\
\hline HE $1135+0139$ & 54871.80 & -2.33 & 0.18 & 1.06 & 0.29 & $\ldots$ & $\ldots$ & $\ldots$ & $\ldots$ & $\ldots$ & $\ldots$ & $\ldots$ & 0.33 & 0.24 \\
\hline HE 1135-0344 & 61544.03 & -2.63 & 0.18 & 0.90 & 0.27 & $\ldots$ & $\ldots$ & $\ldots$ & $\ldots$ & $\ldots$ & $\ldots$ & $\ldots$ & $\cdots$ & \\
\hline HE 1150-0428 & 52002.55 & -3.37 & 0.26 & 2.47 & $\ldots$ & $\ldots$ & $\ldots$ & 2.47 & $\ldots$ & $\ldots$ & $\ldots$ & $\ldots$ & 0.37 & 0.06 \\
\hline HE 1152-0355 & 40001.0 & -1.27 & 0.27 & 0.58 & $\ldots$ & $\ldots$ & $\ldots$ & . & $\ldots$ & $\ldots$ & $\ldots$ & $\ldots$ & -0.01 & $\ldots$ \\
\hline HE 1157-0518 & 49002.00 & -2.34 & 0.31 & 2.15 & 0.29 & 15 & 5 & 1.56 & 0.56 & $\ldots$ & $\ldots$ & $\ldots$ & 0.50 & 0.16 \\
\hline HE 1221-1948 & 60833.81 & -3.36 & 0.18 & 1.29 & 0.31 & $\ldots$ & $\ldots$ & $\ldots$ & $\ldots$ & $\ldots$ & $\ldots$ & $\ldots$ & 0.80 & 0.24 \\
\hline HE 1249-3121 & 53733.40 & -3.23 & 0.19 & 1.73 & 0.27 & $\ldots$ & $\ldots$ & $\ldots$ & $\ldots$ & $\ldots$ & $\ldots$ & $\ldots$ & 0.26 & 0.29 \\
\hline \multirow[t]{3}{*}{ HE $1300+0157$} & 54503.2 & -3.73 & 0.16 & 1.38 & 0.27 & $\ldots$ & $\ldots$ & $\ldots$ & $\ldots$ & (3) 1.76 & 0.39 & $\ldots$ & 0.45 & 0.14 \\
\hline & 54113.38 & -3.76 & 0.19 & 1.04 & 0.29 & $\ldots$ & $\ldots$ & $\ldots$ & $\ldots$ & $\ldots$ & $\ldots$ & $\ldots$ & 0.40 & 0.27 \\
\hline & 56323.37 & -3.46 & 0.19 & 1.33 & 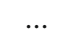 & $\ldots$ & $\ldots$ & $\ldots$ & $\ldots$ & (3) 1.66 & 0.18 & $\ldots$ & 0.35 & 0.10 \\
\hline HE 1300-0641 & 53082.96 & -3.14 & 0.18 & 1.16 & 0.27 & $\ldots$ & $\ldots$ & $\ldots$ & $\ldots$ & $\ldots$ & $\ldots$ & $\ldots$ & 0.04 & 0.26 \\
\hline HE 1300-2201 & 63324.64 & -2.61 & 0.17 & 0.88 & 0.27 & $\ldots$ & $\ldots$ & $\ldots$ & $\ldots$ & $\ldots$ & $\ldots$ & $\ldots$ & 0.29 & 0.24 \\
\hline HE $1305+0007$ & 47502.0 & -2.03 & 0.18 & 1.84 & $\ldots$ & $\ldots$ & $\ldots$ & $\ldots$ & $\ldots$ & $\ldots$ & $\ldots$ & $\ldots$ & 0.25 & $\ldots$ \\
\hline HE 1305-0331 & 60814.22 & -3.26 & 0.18 & 1.00 & 0.29 & $\ldots$ & $\ldots$ & $\ldots$ & $\ldots$ & $\ldots$ & $\ldots$ & $\ldots$ & $\ldots$ & $\ldots$ \\
\hline HE 1319-1935 & 46001.10 & -1.74 & 0.33 & 1.45 & 0.29 & 8 & 3 & 0.46 & 0.56 & & $\ldots$ & $\ldots$ & 0.47 & 0.20 \\
\hline HE $1327-2326$ & 61804.0 & -5.55 & 0.10 & 4.08 & 0.18 & $\ldots$ & $\ldots$ & 4.34 & 0.24 & (3) 3.70 & $\ldots$ & $\ldots$ & 1.71 & 0.05 \\
\hline HE $1327-2326$ & 61804.0 & -5.55 & 0.10 & 4.08 & 0.18 & $\ldots$ & $\ldots$ & 4.34 & 0.24 & (3) 3.70 & $\ldots$ & $\ldots$ & 1.71 & 0.05 \\
\hline HE 1330-0354 & 62574.13 & -2.29 & 0.18 & 0.92 & 0.27 & $\ldots$ & $\ldots$ & $\ldots$ & $\ldots$ & $\ldots$ & $\ldots$ & $\ldots$ & 0.32 & 0.23 \\
\hline HE 1351-1049 & 52042.85 & -3.46 & 0.18 & 1.42 & 0.28 & $\ldots$ & $\ldots$ & $\ldots$ & $\ldots$ & $\ldots$ & $\ldots$ & $\ldots$ & 0.30 & 0.27 \\
\hline HE 1410-0004 & 49852.00 & -3.09 & 0.17 & 2.09 & $\ldots$ & $\ldots$ & $\ldots$ & $\ldots$ & $\ldots$ & (2) 1.26 & $\ldots$ & $\mathrm{NaN}$ & 0.61 & 0.29 \\
\hline \multirow[t]{2}{*}{ HE $1410+0213$} & 48002.00 & -2.52 & 0.21 & 2.33 & 0.10 & 3 & 0.5 & 2.94 & 0.10 & 2.56 & 0.20 & $\ldots$ & 0.33 & 0.18 \\
\hline & 56053.50 & -2.23 & 0.31 & 1.83 & & 3 & 1 & 1.73 & $\ldots$ & $\ldots$ & $\ldots$ & $\ldots$ & 0.21 & 0.03 \\
\hline HE 1413-1954 & 65334.59 & -3.22 & 0.17 & 1.32 & 0.32 & $\ldots$ & $\ldots$ & $\ldots$ & $\ldots$ & $\ldots$ & $\ldots$ & $\ldots$ & $\ldots$ & $\cdots$ \\
\hline HE 1419-1324 & 49001.80 & -3.05 & 0.29 & 1.76 & 0.10 & 12 & 2 & 1.47 & 0.10 & $<1.19$ & $\ldots$ & $\ldots$ & 0.53 & 0.13 \\
\hline HE 1429-0551 & 47001.50 & -2.47 & 0.20 & 2.28 & 0.19 & 30 & 15 & 1.39 & 0.52 & $\ldots$ & $\ldots$ & $\ldots$ & 0.52 & 0.15 \\
\hline HE $1430-1123$ & 59153.75 & -2.71 & 0.18 & 1.71 & 0.28 & $\ldots$ & $\ldots$ & $\ldots$ & $\ldots$ & $\ldots$ & $\ldots$ & $\ldots$ & 0.35 & 0.24 \\
\hline HE $1434-1442$ & 54203.15 & -2.46 & 0.18 & 2.05 & $\ldots$ & $\ldots$ & $\ldots$ & 1.35 & $\ldots$ & $\ldots$ & $\ldots$ & $\ldots$ & 0.33 & 0.05 \\
\hline HE $1443+0113$ & 49451.85 & -2.14 & 0.21 & 1.94 & $\ldots$ & $\ldots$ & $\ldots$ & $\ldots$ & $\ldots$ & $\ldots$ & $\ldots$ & $\ldots$ & 0.40 & 0.09 \\
\hline HE $1447+0102$ & 51001.70 & -2.47 & 0.27 & 2.48 & 0.20 & 25 & 10 & 1.39 & 0.44 & $\ldots$ & $\ldots$ & $\ldots$ & 1.43 & 0.13 \\
\hline HE 1509-0806 & 51852.50 & -2.98 & 0.16 & 2.08 & $\ldots$ & $\ldots$ & $\ldots$ & 2.18 & $\ldots$ & $\ldots$ & $\ldots$ & $\ldots$ & 0.67 & 0.11 \\
\hline HE 1523-1155 & 48001.60 & -2.15 & 0.19 & 1.86 & 0.19 & $\ldots$ & $\ldots$ & 1.67 & 0.52 & $\ldots$ & $\ldots$ & $\ldots$ & 0.62 & 0.15 \\
\hline HE 1528-0409 & 50001.80 & -2.61 & 0.19 & 2.42 & 0.19 & 12 & 5 & 2.03 & 0.52 & $\ldots$ & $\ldots$ & $\ldots$ & 0.83 & 0.13 \\
\hline HE 2148-1247 & 63803.9 & -2.37 & 0.20 & 1.93 & $\ldots$ & 10 & 3 & 1.70 & 0.30 & $\ldots$ & $\ldots$ & $\ldots$ & 0.51 & 0.25 \\
\hline HE 2150-0825 & 59603.67 & -1.98 & 0.18 & 1.22 & 0.27 & $\ldots$ & $\ldots$ & $\ldots$ & $\ldots$ & $\ldots$ & $\ldots$ & $\ldots$ & 0.36 & 0.24 \\
\hline \multirow[t]{2}{*}{ HE 2158-0348 } & 57923.6 & -2.07 & 0.06 & 2.57 & 0.10 & 20 & 10 & 1.82 & 0.20 & $\ldots$ & $\ldots$ & $\ldots$ & 0.18 & 0.05 \\
\hline & 52152.50 & -2.77 & 0.26 & 1.97 & & 4 & 1 & 1.47 & $\ldots$ & $\ldots$ & $\ldots$ & $\ldots$ & 0.71 & 0.18 \\
\hline HE 2221-0453 & 44000.40 & -2.22 & 0.31 & 1.83 & 0.31 & 10 & 4 & 0.84 & 0.48 & $\ldots$ & $\ldots$ & $\ldots$ & 0.80 & 0.17 \\
\hline
\end{tabular}


Table 1. continued.

\begin{tabular}{|c|c|c|c|c|c|c|c|c|c|c|c|c|c|c|}
\hline $\begin{array}{l}\text { Object } \\
(1)\end{array}$ & $\begin{array}{c}T_{\text {eff }} \log g \\
(2)\end{array}$ & $\begin{array}{c}\mathrm{Fe} / \mathrm{H}] \\
(3)\end{array}$ & $\begin{array}{c}\sigma \\
(4) \\
\end{array}$ & $\begin{array}{c}\mathrm{C} / \mathrm{Fe}] \\
(5) \\
\end{array}$ & $\begin{array}{c}\sigma \\
(6) \\
\end{array}$ & $\begin{array}{c}{ }^{{ }^{12} \mathrm{C} /{ }^{13} \mathrm{C}} \\
(7)\end{array}$ & $\begin{array}{c}\sigma \\
(8) \\
\end{array}$ & $\begin{array}{c}{[\mathrm{N} / \mathrm{Fe}]} \\
(9) \\
\end{array}$ & $\begin{array}{c}\sigma \\
(10) \\
\end{array}$ & $\begin{array}{c}\mathrm{O} / \mathrm{Fe}] \\
(11) \\
\end{array}$ & $\begin{array}{c}\sigma \\
(12) \\
\end{array}$ & $\begin{array}{c}\text { Non-LTE } \\
\text { (13) }\end{array}$ & $\begin{array}{c}{[\mathrm{Mg} / \mathrm{Fe}]} \\
(14)\end{array}$ & $\begin{array}{c}\sigma \\
(15) \\
\end{array}$ \\
\hline HE 2227-4044 & 58113.85 & -2.32 & 0.17 & 1.54 & 0.26 & $\ldots$ & $\ldots$ & $\ldots$ & & $\ldots$ & $\ldots$ & $\ldots$ & 0.30 & 0.23 \\
\hline HE 2228-0706 & 51002.60 & -2.41 & 0.20 & 2.32 & 0.19 & 15 & 4 & 1.13 & 0.33 & $\ldots$ & $\ldots$ & $\ldots$ & 0.67 & 0.15 \\
\hline \multirow[t]{2}{*}{ HE 2232-0603 } & 64004.1 & -1.00 & 0.11 & 1.62 & 0.10 & 30 & 2 & 0.42 & 0.20 & $\ldots$ & $\ldots$ & $\ldots$ & 0.30 & 0.12 \\
\hline & 57503.50 & -1.92 & 0.23 & 1.32 & & $>30$ & $\ldots$ & 0.42 & $\ldots$ & $\ldots$ & $\ldots$ & $\ldots$ & 0.88 & 0.32 \\
\hline HE 2240-0412 & 58524.33 & -2.20 & 0.18 & 1.22 & 0.26 & $\ldots$ & $\ldots$ & $\ldots$ & $\ldots$ & $\ldots$ & $\ldots$ & $\ldots$ & 0.28 & 0.24 \\
\hline HE 2330-0555 & 49001.70 & -2.78 & 0.19 & 2.09 & 0.19 & $\ldots$ & $\ldots$ & 1.00 & 0.42 & $\ldots$ & $\ldots$ & $\ldots$ & 0.67 & 0.17 \\
\hline CS 22183-015 & 52002.5 & -3.12 & 0.19 & 2.20 & & $\ldots$ & $\ldots$ & $\ldots$ & ... & $\ldots$ & $\ldots$ & $\ldots$ & $\ldots$ & $\ldots$ \\
\hline \multirow[t]{3}{*}{ or HE $0058-0244$} & 54702.85 & -2.85 & 0.02 & 2.34 & 0.33 & $\ldots$ & $\ldots$ & 1.79 & 0.23 & $\ldots$ & $\ldots$ & $\ldots$ & 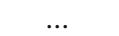 & \\
\hline & 56203.4 & -2.75 & 0.21 & 1.95 & $\ldots$ & 9 & 1 & 1.78 & 0.20 & $\ldots$ & $\ldots$ & $\ldots$ & 0.87 & 0.19 \\
\hline & 57333.6 & -2.37 & 0.10 & 2.42 & 0.10 & 8 & 2 & 1.92 & 0.20 & $\ldots$ & $\ldots$ & $\ldots$ & 0.37 & 0.20 \\
\hline CS 22877-001 & 51002.2 & -2.72 & 0.19 & 1.00 & 0.18 & $>10$ & $\ldots$ & $\ldots$ & 0.24 & $\ldots$ & $\ldots$ & $\ldots$ & 0.29 & 0.08 \\
\hline \multirow[t]{2}{*}{ CS 22880-074 } & 58503.8 & -1.93 & 0.11 & 1.30 & 0.38 & $>40$ & $\ldots$ & -0.10 & $\ldots$ & $\ldots$ & $\ldots$ & $\ldots$ & 0.46 & 0.19 \\
\hline & 60504.0 & -1.80 & $\ldots$ & 1.51 & $\ldots$ & $>40$ & $\ldots$ & 0.20 & $\ldots$ & $\ldots$ & $\ldots$ & $\ldots$ & 0.06 & $\ldots$ \\
\hline CS 22881-036 & 62004.0 & -2.10 & $\ldots$ & 1.96 & $\ldots$ & 40 & $\ldots$ & 1.00 & $\ldots$ & $\ldots$ & $\ldots$ & $\ldots$ & 0.40 & $\ldots$ \\
\hline CS 22887-048 & 65003.35 & -1.70 & 0.04 & 1.84 & 0.23 & $\ldots$ & $\ldots$ & $\ldots$ & $\ldots$ & $\ldots$ & $\ldots$ & $\ldots$ & $\ldots$ & $\ldots$ \\
\hline CS 22891-171 & 51001.60 & -2.25 & 0.17 & 1.56 & 0.10 & 6 & 2 & 1.67 & 0.10 & $<0.79$ & 0.00 & $\ldots$ & 0.70 & 0.37 \\
\hline \multirow[t]{3}{*}{ CS 22892-052 } & 47101.5 & -3.10 & 0.13 & 1.05 & 0.10 & 15 & 2 & 1.00 & 0.20 & 0.72 & 0.15 & $\ldots$ & 0.30 & 0.08 \\
\hline & 48501.6 & -3.03 & 0.14 & 0.92 & 0.06 & 16 & 7 & 0.51 & 0.13 & 0.41 & 0.12 & $\ldots$ & 0.22 & 0.13 \\
\hline & 48841.81 & -2.95 & 0.19 & 0.87 & 0.28 & $\ldots$ & $\ldots$ & $\ldots$ & $\ldots$ & $\ldots$ & $\ldots$ & $\ldots$ & 0.12 & 0.24 \\
\hline \multirow[t]{3}{*}{ CS 22898-027 } & 62503.7 & -2.26 & 0.11 & 2.20 & 0.38 & 15 & 5 & 0.90 & 0.43 & $\ldots$ & $\ldots$ & $\ldots$ & 0.41 & 0.19 \\
\hline & 62403.72 & -2.30 & 0.02 & 2.34 & 0.04 & $\ldots$ & $\ldots$ & 1.24 & 0.07 & $\ldots$ & $\ldots$ & $\ldots$ & $\ldots$ & $\ldots$ \\
\hline & 63004.0 & -2.00 & & 1.95 & & $>20$ & $\ldots$ & 1.20 & $\ldots$ & $\ldots$ & $\ldots$ & $\ldots$ & 0.13 & 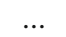 \\
\hline \multirow[t]{3}{*}{ CS 22942-019 } & 51002.50 & -2.43 & 0.15 & 2.14 & 0.10 & 12 & 1 & 1.15 & 0.10 & 0.97 & 0.20 & $\ldots$ & 0.60 & 0.21 \\
\hline & 50002.4 & -2.64 & 0.14 & 2.00 & 0.24 & 8 & 2 & 0.80 & 0.37 & $\ldots$ & $\ldots$ & $\ldots$ & 0.58 & 0.22 \\
\hline & 49001.8 & -2.67 & 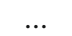 & 2.20 & $\ldots$ & 30 & 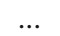 & 0.70 & 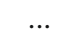 & $\ldots$ & $\ldots$ & $\ldots$ & 0.77 & 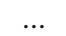 \\
\hline CS 22945-017 & 64003.80 & -2.52 & 0.12 & 2.28 & 0.10 & 6 & 3 & 2.24 & 0.10 & $<2.36$ & $\ldots$ & $\ldots$ & 0.61 & 0.10 \\
\hline \multirow[t]{4}{*}{ CS 22948-027 } & 48001.8 & -2.47 & $\ldots$ & 2.43 & $\ldots$ & $\ldots$ & $\ldots$ & 1.75 & $\ldots$ & $\ldots$ & $\ldots$ & $\ldots$ & 0.31 & 0.28 \\
\hline & 46000.8 & -2.60 & $\ldots$ & 2.10 & $\ldots$ & 20 & $\ldots$ & 1.10 & $\ldots$ & $\ldots$ & $\ldots$ & $\ldots$ & 0.35 & $\ldots$ \\
\hline & 46001.0 & -2.57 & 0.23 & 2.00 & 0.18 & 10 & $\ldots$ & 1.80 & 0.24 & $\ldots$ & $\ldots$ & $\ldots$ & 0.68 & 0.20 \\
\hline & 50001.90 & -2.21 & 0.28 & 2.12 & 0.20 & 10 & 3 & 2.43 & 0.35 & $\ldots$ & $\ldots$ & $\ldots$ & 0.55 & 0.14 \\
\hline CS 22949-037 & 49001.5 & -3.97 & 0.11 & 1.17 & 0.10 & 3 & 1 & 2.57 & 0.20 & $<1.98$ & 0.10 & 1.00 & 1.58 & 0.19 \\
\hline or HE 2323-0256 & 49151.7 & -3.86 & 0.16 & 1.07 & $\ldots$ & $\ldots$ & $\ldots$ & 2.11 & $\ldots$ & (3) 1.93 & $\ldots$ & $\ldots$ & 1.50 & 0.25 \\
\hline \multirow[t]{3}{*}{ CS 22956-028 } & 67003.50 & -2.33 & 0.09 & 1.84 & 0.10 & 5 & 2 & 1.85 & 0.10 & $<2.47$ & $\ldots$ & $\ldots$ & 0.58 & 0.09 \\
\hline & 70384.3 & -1.89 & 0.06 & 1.82 & 0.10 & 5 & 2 & 1.52 & 0.20 & (2) 0.63 & 0.11 & $\mathrm{NaN}$ & 0.31 & 0.05 \\
\hline & 69003.9 & -2.08 & $\ldots$ & 1.60 & 0.20 & $\ldots$ & $\ldots$ & $\ldots$ & $\ldots$ & (2) 0.50 & 0.12 & -0.14 & 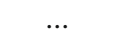 & 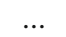 \\
\hline CS 22957-027 & 48501.9 & -3.36 & 0.13 & 2.22 & 0.30 & 10 & 5 & 2.02 & 0.50 & $\ldots$ & $\ldots$ & $\ldots$ & 0.67 & 0.06 \\
\hline or HE 2356-0410 & 48392.25 & -3.43 & 0.12 & 2.00 & 0.20 & 10 & $\ldots$ & 1.10 & 0.20 & $\ldots$ & $\ldots$ & $\ldots$ & $\cdots$ & $\ldots$ \\
\hline & 50502.0 & -2.96 & $\ldots$ & 2.20 & $\ldots$ & 25 & $\ldots$ & 1.60 & $\cdots$ & $\ldots$ & $\ldots$ & $\ldots$ & 0.21 & $\ldots$ \\
\hline & 51001.9 & -3.12 & 0.15 & 2.37 & 0.24 & 8 & 2 & 1.62 & 0.35 & $\ldots$ & $\ldots$ & $\ldots$ & 0.69 & 1.60 \\
\hline & 52052.5 & -3.14 & 0.21 & 2.24 & & 4 & 1 & 1.84 & & $\ldots$ & $\ldots$ & $\ldots$ & 0.39 & 0.50 \\
\hline & 53643.1 & -2.69 & 0.06 & 2.17 & 0.05 & 15 & 10 & 1.72 & 0.20 & $\ldots$ & $\ldots$ & $\ldots$ & -0.03 & 0.20 \\
\hline CS 22958-042 & 62503.5 & -2.85 & 0.10 & 3.17 & 0.12 & 9 & 2 & 2.17 & 0.12 & (2) 1.35 & 0.11 & -0.12 & 0.37 & 0.15 \\
\hline CS 22960-053 & 52002.10 & -3.14 & 0.15 & 2.05 & 0.16 & $\ldots$ & $\ldots$ & 3.06 & 0.21 & $\ldots$ & $\ldots$ & $\ldots$ & 0.65 & 0.12 \\
\hline CS 22967-007 & 64794.2 & -1.79 & 0.06 & 1.82 & 0.10 & $>60$ & & 0.92 & 0.20 & (2) 0.90 & 0.10 & $\mathrm{NaN}$ & 0.61 & 0.06 \\
\hline CS 29495-042 & 55443.4 & -1.86 & 0.06 & 1.32 & 0.10 & 7 & 2 & 1.32 & 0.10 & (2) 0.59 & $\ldots$ & $\mathrm{NaN}$ & 0.67 & 0.12 \\
\hline CS 29497-030 & 66503.5 & -2.70 & 0.14 & 2.38 & ... & $>10$ & $\ldots$ & 1.88 & $\ldots$ & (2) 1.68 & 0.10 & -0.16 & 0.54 & 0.21 \\
\hline & 70004.10 & -2.57 & $\ldots$ & 2.47 & 0.10 & $\ldots$ & $\ldots$ & 2.12 & 0.35 & (2) 1.48 & 0.11 & $\mathrm{NaN}$ & 0.44 & 0.14 \\
\hline CS 29497-034 & 48001.8 & -2.90 & $\ldots$ & 2.63 & & $\ldots$ & $\ldots$ & 2.38 & & $\ldots$ & $\ldots$ & $\ldots$ & 0.72 & 0.34 \\
\hline & 49832.1 & -2.55 & 0.14 & 2.42 & 0.20 & 20 & 5 & 2.32 & 0.30 & $<0.92$ & 0.20 & $\ldots$ & 0.27 & 0.20 \\
\hline or HE 0039-2635 & 49001.50 & -2.91 & 0.27 & 2.72 & 0.20 & $\ldots$ & $\ldots$ & 2.63 & 0.53 & $\ldots$ & $\ldots$ & $\ldots$ & 1.31 & 0.12 \\
\hline CS 29498-043 & 46001.2 & -3.54 & 0.28 & 2.09 & 0.29 & 6 & 2 & 2.27 & 0.40 & 2.43 & 0.11 & $\mathrm{NaN}$ & 1.75 & 0.25 \\
\hline & 44000.6 & -3.75 & 0.26 & 1.90 & 0.29 & 6 & 2 & 2.30 & 0.40 & $\ldots$ & $\ldots$ & $\ldots$ & 1.81 & 0.24 \\
\hline CS 29502-092 & 50002.1 & -2.76 & 0.18 & 1.00 & 0.18 & 20 & $\ldots$ & 0.70 & 0.24 & $\ldots$ & $\ldots$ & $\ldots$ & 0.37 & 0.07 \\
\hline & 49701.70 & -3.05 & 0.02 & 1.18 & 0.23 & $\ldots$ & $\ldots$ & 1.28 & 0.17 & $\ldots$ & $\ldots$ & $\ldots$ & $\ldots$ & $\ldots$ \\
\hline CS 29503-010 & 65004.50 & -1.06 & 0.19 & 1.07 & 0.19 & $\ldots$ & $\ldots$ & $\ldots$ & $\ldots$ & $\ldots$ & $\ldots$ & $\ldots$ & 0.36 & 0.18 \\
\hline CS 29509-027 & 70504.2 & -2.02 & $\ldots$ & 1.24 & 0.20 & $\ldots$ & $\ldots$ & $\ldots$ & $\ldots$ & (2) 0.55 & 0.17 & $\mathrm{NaN}$ & $\cdots$ & $\ldots$ \\
\hline CS 29526-110 & 65003.2 & -2.38 & 0.16 & 2.20 & 0.38 & $\ldots$ & $\ldots$ & 1.40 & $\ldots$ & $\ldots$ & $\ldots$ & $\ldots$ & 0.30 & 0.19 \\
\hline CS 29528-028 & 68004.00 & -2.86 & 0.22 & 2.77 & 0.21 & $\ldots$ & $\ldots$ & $\ldots$ & $\ldots$ & $\ldots$ & $\ldots$ & $\ldots$ & 1.69 & 0.21 \\
\hline CS 29528-041 & 61504.0 & -3.30 & 0.10 & 1.61 & 0.15 & $\ldots$ & $\ldots$ & 3.09 & 0.13 & $<1.40$ & 0.10 & $\ldots$ & 0.45 & 0.11 \\
\hline CS 30301-015 & 47500.8 & -2.64 & 0.18 & 1.60 & 0.28 & 6 & 2 & 1.70 & 0.43 & $\ldots$ & $\ldots$ & $\ldots$ & 0.86 & 0.22 \\
\hline CS 30314-067 & 44000.7 & -2.85 & 0.18 & 0.50 & 0.18 & 5 & $\ldots$ & 1.20 & 0.24 & $\ldots$ & $\ldots$ & $\ldots$ & 0.42 & 0.10 \\
\hline CS 30315-091 & 55363.4 & -1.66 & 0.06 & 1.32 & 0.10 & $>60$ & $\ldots$ & 0.42 & 0.20 & (2) 0.86 & 0.11 & $\mathrm{NaN}$ & 0.64 & 0.07 \\
\hline CS 30322-023 & $4100-0.30$ & -3.39 & 0.18 & 0.80 & 0.10 & 4 & 1 & 2.91 & 0.10 & 0.63 & 0.20 & $\ldots$ & 0.80 & 0.09 \\
\hline & 43001.00 & -3.25 & 0.23 & 0.56 & 0.12 & $\ldots$ & $\ldots$ & 2.47 & 0.33 & $\ldots$ & $\ldots$ & $\ldots$ & 0.54 & 0.26 \\
\hline CS 30323-107 & 61264.4 & -1.73 & 0.06 & 1.12 & 0.10 & 9 & 2 & 0.82 & 0.20 & (2) 0.79 & 0.10 & $\mathrm{NaN}$ & 0.52 & 0.12 \\
\hline CS 30338-089 & 50002.10 & -2.45 & 0.20 & 2.06 & 0.19 & 12 & 4 & 1.27 & 0.33 & $\ldots$ & $\ldots$ & $\ldots$ & 0.48 & 0.14 \\
\hline
\end{tabular}


T. Masseron et al.: A holistic approach to carbon-enhanced metal-poor stars

Table 1. continued.

\begin{tabular}{|c|c|c|c|c|c|c|c|c|c|c|c|c|c|c|}
\hline $\begin{array}{l}\text { Object } \\
\text { (1) }\end{array}$ & $\begin{array}{c}T_{\text {eff }} \log g \\
\text { (2) }\end{array}$ & $\begin{array}{c}{[\mathrm{Fe} / \mathrm{H}]} \\
(3)\end{array}$ & $\begin{array}{c}\sigma \\
(4)\end{array}$ & $\begin{array}{c}\mathrm{C} / \mathrm{Fe}] \\
(5)\end{array}$ & $\begin{array}{c}\sigma \\
(6)\end{array}$ & $\begin{array}{c}{ }^{12} \mathrm{C} /{ }^{13} \mathrm{C} \\
(7)\end{array}$ & $\begin{array}{c}\sigma \\
(8)\end{array}$ & $\begin{array}{c}{[\mathrm{N} / \mathrm{Fe}]} \\
(9)\end{array}$ & $\begin{array}{c}\sigma \\
(10)\end{array}$ & $\begin{array}{c}\mathrm{O} / \mathrm{Fe}] \\
(11)\end{array}$ & $\begin{array}{c}\sigma \\
(12)\end{array}$ & $\begin{array}{c}\text { Non-LTE } \\
\text { (13) }\end{array}$ & $\begin{array}{c}\mathrm{Mg} / \mathrm{Fe}] \\
\text { (14) }\end{array}$ & $\begin{array}{c}\sigma \\
(15)\end{array}$ \\
\hline CS 30338-089 & 52022.6 & -1.73 & 0.14 & 1.52 & 0.10 & 8 & 5 & 0.82 & 0.30 & 0.75 & 0.10 & $\ldots$ & 0.22 & \\
\hline \multirow{2}{*}{ CS 31062-050 } & 56003.0 & -2.31 & 0.14 & 2.00 & 0.24 & 8 & 2 & 1.20 & 0.37 & $\ldots$ & $\ldots$ & $\ldots$ & 0.60 & 0.21 \\
\hline & 55002.70 & -2.41 & 0.11 & 1.82 & 0.00 & $\ldots$ & $\ldots$ & & $\ldots$ & 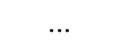 & $\ldots$ & $\ldots$ & 0.84 & 0.20 \\
\hline CS 31080-095 & 60504.5 & -2.85 & 0.10 & 2.71 & 0.14 & $>40$ & $\ldots$ & 0.72 & 0.11 & (2) 2.35 & 0.12 & $\mathrm{NaN}$ & 0.70 & 0.12 \\
\hline BS 16080-175 & 62403.70 & -1.86 & 0.04 & 1.75 & 0.23 & $\cdots$ & $\ldots$ & $\cdots$ & $\cdots$ & $\cdots$ & $\cdots$ & $\ldots$ & 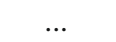 & \\
\hline BS 16929-005 & 52502.80 & -3.17 & 0.14 & 1.08 & 0.16 & $\ldots$ & $\ldots$ & $\ldots$ & $\ldots$ & $\ldots$ & $\ldots$ & $\ldots$ & 0.49 & 0.10 \\
\hline BS $17436-058$ & 56902.68 & -1.78 & 0.04 & 1.50 & 0.24 & $\ldots$ & $\ldots$ & 1.25 & 0.23 & & 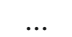 & $\ldots$ & $\ldots$ & 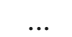 \\
\hline HD 26 & 49001.50 & -1.02 & 0.22 & 0.68 & 0.10 & 9 & 2 & 0.94 & 0.10 & 0.36 & 0.20 & $\ldots$ & 0.93 & 0.27 \\
\hline HD 5223 & 45001.0 & -2.06 & 0.13 & 1.57 & $\cdots$ & $\ldots$ & $\ldots$ & $\ldots$ & $\ldots$ & $\ldots$ & $\ldots$ & $\ldots$ & 0.58 & $\ldots$ \\
\hline HD 187861 & 46001.70 & -2.36 & 0.21 & 2.02 & 0.10 & 10 & 2 & 2.18 & 0.10 & 1.40 & 0.20 & $\ldots$ & 0.37 & 0.07 \\
\hline \multirow[t]{3}{*}{ HD 196944} & 52501.70 & -2.19 & 0.13 & 1.30 & 0.10 & 5 & 1 & 1.41 & 0.10 & 0.63 & 0.20 & $\ldots$ & 0.56 & 0.09 \\
\hline & 52501.8 & -2.25 & 0.19 & 1.20 & 0.24 & 5 & 1 & 1.30 & 0.37 & $\cdots$ & $\ldots$ & $\ldots$ & 0.42 & 0.21 \\
\hline & 52501.7 & -2.46 & 0.10 & 1.42 & & $\ldots$ & $\ldots$ & $\cdots$ & $\cdots$ & (2) 1.11 & 0.05 & $\mathrm{NaN}$ & 0.40 & 0.07 \\
\hline HD 206983 & 42000.60 & -0.99 & 0.14 & 0.50 & 0.10 & 5 & 3 & 1.21 & 0.10 & $<0.23$ & ... & $\ldots$ & 0.26 & 0.31 \\
\hline HD 224959 & 49002.00 & -2.06 & 0.20 & 1.77 & 0.10 & 4 & 2 & 1.88 & 0.10 & 1.10 & 0.20 & $\ldots$ & 0.76 & 0.24 \\
\hline HKII17435-00532 & $5200,2.15$ & -2.23 & 0.23 & 0.68 & 0.30 & $\ldots$ & $\ldots$ & $\ldots$ & $\ldots$ & (2) 1.16 & 0.27 & $\mathrm{NaN}$ & 0.42 & 0.25 \\
\hline \multirow[t]{2}{*}{ LP 625-44 } & 55002.8 & -2.71 & 0.13 & 2.10 & & 20 & $\ldots$ & 1.00 & 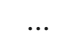 & & & $\ldots$ & $\cdots$ & 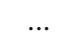 \\
\hline & 55002.50 & -2.72 & 0.20 & 2.25 & 0.23 & $\ldots$ & $\ldots$ & 0.95 & 0.35 & (2) 1.85 & 0.20 & -0.15 & 1.12 & 0.24 \\
\hline LP 706-7 & 60003.8 & -2.74 & 0.16 & 2.15 & 0.23 & $\ldots$ & $\ldots$ & 1.80 & 0.35 & $\ldots$ & $\cdots$ & $\ldots$ & $\ldots$ & $\cdots$ \\
\hline or CS 31062-012 & 62504.5 & -2.55 & 0.11 & 2.10 & 0.38 & 15 & 5 & 1.20 & 0.43 & $\ldots$ & $\ldots$ & $\ldots$ & 0.45 & 0.20 \\
\hline G 77-61 & 40005.0 & -4.03 & 0.15 & 2.49 & 0.10 & 5 & 1 & 2.48 & 0.10 & 2.11 & 0.20 & $\ldots$ & 0.49 & 0.20 \\
\hline V 5430ph & 62501.5 & -2.15 & 0.12 & -0.29 & $\ldots$ & $\ldots$ & $\ldots$ & $\ldots$ & $\ldots$ & (2) 0.94 & 0.07 & -0.27 & 0.13 & 0.08 \\
\hline
\end{tabular}


Table 2. Abundances of neutron-capture elements in carbon enhanced metal-poor stars from literature. Columns (17), (19), (21), (23), (25) list random errors (when available). (27) Solar abundances reference adopted in the original reference source: Anders \& Grevesse (1989, An89), Grevesse et al. (1996, Gr96), Grevesse \& Sauval (1998, Gr98), Grevesse et al. (2000, Gr20), Asplund (2005, As05). references: (1) Cohen et al. (2004), (2) Cohen et al. (2006), (3) Lucatello et al. (2003), (4) Aoki et al. (2007), (5) Barklem et al. (2005), (6) Lucatello (2003), (7) Christlieb et al. (2002), (8) Christlieb et al. (2004), (9) Jonsell et al. (2006), (10) Norris et al. (2007), (11) Cohen et al. (2008), (12) Goswami et al. (2006), (13) Frebel et al. (2007), (14) Frebel et al. (2005), (15) Aoki et al. (2006), (16) Frebel et al. (2006), (17) Cohen et al. (2003), (18) Johnson \& Bolte (2002), (19) Tsangarides (2005), (20) Aoki et al. (2002c), (21) Aoki et al. (2002d), (22) Aoki et al. (2002b), (23) Preston \& Sneden (2001), (24) Sneden et al. (2003a), (25) Cayrel et al. (2004), (26) Spite et al. (2005), (27) Spite et al. (2006), (28) Barbuy et al. (2005), (29) François et al. (2007), (30) Depagne et al. (2002), (31) Sneden et al. (2003b), (32) Norris et al. (1997), (33) Bonifacio et al. (1998), (34) Aoki et al. (2002a), (35) Sivarani et al. (2006), (36) Sivarani et al. (2004), (37) Ivans et al. (2005), (38) Aoki et al. (2004), (39) Johnson \& Bolte (2004), (40) Zacs et al. (1998), (41) Aoki et al. (2001), (43) Plez \& Cohen (2005), (44) Deroo et al. (2005), (45) Honda et al. (2004), (46) Allen \& Barbuy (2006a), (47) Christlieb et al. (2004), (48) Hill et al. (2002), (49) Plez et al. (2004), (50) Honda et al. (2006), (51) Roederer et al. (2008), (52) Paper II.

\begin{tabular}{|c|c|c|c|c|c|c|c|c|c|c|c|c|c|}
\hline Object & $\begin{array}{c}{[\mathrm{Ba} / \mathrm{Fe}]} \\
(16)\end{array}$ & $\begin{array}{c}\sigma \\
(17)\end{array}$ & $\begin{array}{c}{[\mathrm{La} / \mathrm{Fe}]} \\
(18)\end{array}$ & $\begin{array}{c}\sigma \\
(19)\end{array}$ & $\begin{array}{c}{[\mathrm{Ce} / \mathrm{Fe}]} \\
(20)\end{array}$ & $\begin{array}{c}\sigma \\
(21)\end{array}$ & $\begin{array}{c}{[\mathrm{Eu} / \mathrm{Fe}]} \\
(22)\end{array}$ & $\begin{array}{c}\sigma \\
(23)\end{array}$ & $\begin{array}{c}{[\mathrm{Pb} / \mathrm{Fe}]} \\
(24)\end{array}$ & $\begin{array}{c}\sigma \\
(25)\end{array}$ & $\begin{array}{c}\text { Class } \\
(26)\end{array}$ & $\begin{array}{c}\text { Solar ref } \\
\text { (27) }\end{array}$ & $\begin{array}{l}\text { References } \\
(28)\end{array}$ \\
\hline HE 0007-1832 & 0.23 & & $<1.80$ & $\ldots$ & $\ldots$ & $\ldots$ & $<1.86$ & $\ldots$ & $<3.21$ & $\ldots$ & no & An89 & (1) \\
\hline HE 0012-1441 & 1.22 & 0.27 & $\ldots$ & $\ldots$ & $\ldots$ & $\ldots$ & $\ldots$ & $\ldots$ & $<2.09$ & $\ldots$ & $\mathrm{s}$ & An89 & (2) \\
\hline HE 0024-2523 & 1.52 & 0.20 & 1.77 & 0.20 & $\ldots$ & $\ldots$ & $<1.07$ & 0.10 & 3.32 & 0.10 & $\mathrm{~s}$ & An89 & (3) \\
\hline HE 0107-5240 & $<0.82$ & $\ldots$ & $\ldots$ & $\ldots$ & $\ldots$ & $\ldots$ & $<2.80$ & 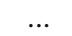 & $\ldots$ & $\ldots$ & no & Gr98 & $(7,8)$ \\
\hline HE 0131-3953 & 2.20 & 0.23 & 1.94 & 0.27 & 1.93 & 0.29 & 1.62 & 0.28 & $\ldots$ & $\ldots$ & rs & Gr98 & (5) \\
\hline \multirow[t]{2}{*}{ HE 0143-0441 } & 2.39 & 0.12 & 1.77 & 0.15 & 2.00 & 0.21 & 1.53 & 0.17 & 3.28 & $\ldots$ & rs & An89 & (2) \\
\hline & 2.38 & 0.18 & 1.96 & 0.14 & 2.20 & 0.21 & 1.72 & 0.17 & 3.67 & $\ldots$ & rs & An89 & (1) \\
\hline HE 0202-2204 & 1.41 & 0.22 & 1.36 & 0.34 & 1.30 & 0.26 & 0.49 & 0.24 & $\ldots$ & $\ldots$ & $\mathrm{s}$ & Gr98 & (5) \\
\hline HE 0206-1916 & 1.97 & 0.16 & & & & $\ldots$ & $\ldots$ & $\ldots$ & $\ldots$ & $\ldots$ & $\mathrm{s}$ & As05 & (4) \\
\hline HE 0212-0557 & 2.25 & 0.06 & 2.27 & 0.22 & 2.21 & & $\ldots$ & $\ldots$ & $\ldots$ & $\ldots$ & rs & An89 & (2) \\
\hline HE 0231-4016 & 1.47 & 0.23 & 1.22 & 0.28 & 1.53 & 0.27 & $\ldots$ & $\ldots$ & $\ldots$ & $\ldots$ & $\mathrm{s}$ & Gr98 & (5) \\
\hline \multirow[t]{2}{*}{ HE $0336+0113$} & 2.12 & & 2.07 & 0.10 & 1.87 & 0.19 & 1.47 & 0.20 & 2.82 & 0.20 & rs & An89 & (6) \\
\hline & 2.70 & 0.32 & 1.92 & 0.14 & 2.37 & 0.18 & 1.25 & 0.13 & $<2.45$ & $\ldots$ & rs & An89 & (2) \\
\hline HE 0338-3945 & 2.41 & 0.07 & 2.28 & 0.16 & 2.16 & 0.18 & 1.94 & 0.17 & 3.10 & 0.11 & rs & As05 & (9) \\
\hline HE 0400-2030 & 1.64 & 0.16 & $\ldots$ & $\ldots$ & $\ldots$ & $\ldots$ & $\ldots$ & $\ldots$ & $\ldots$ & $\ldots$ & $\mathrm{s}$ & As05 & (4) \\
\hline HE 0430-4404 & 1.62 & 0.23 & 1.41 & 0.26 & $\ldots$ & $\ldots$ & $\ldots$ & $\ldots$ & $\ldots$ & $\ldots$ & $\mathrm{s}$ & Gr98 & (5) \\
\hline HE 0441-0652 & 1.11 & 0.24 & $\ldots$ & $\ldots$ & $\ldots$ & $\ldots$ & $\ldots$ & $\ldots$ & $\ldots$ & $\ldots$ & $\mathrm{s}$ & As05 & (4) \\
\hline HE 0507-1653 & 1.89 & 0.14 & $\ldots$ & $\ldots$ & $\ldots$ & $\ldots$ & $\ldots$ & $\ldots$ & $\ldots$ & $\ldots$ & $\mathrm{s}$ & As05 & (4) \\
\hline HE 0557-4840 & $<0.03$ & $\ldots$ & $\ldots$ & $\ldots$ & $\ldots$ & $\ldots$ & $<2.04$ & $\ldots$ & $\ldots$ & $\ldots$ & no & As05 & (10) \\
\hline HE 1001-0243 & 0.41 & 0.20 & 0.55 & 0.20 & 0.80 & 0.10 & -0.04 & 0.20 & $<1.38$ & $\ldots$ & low-s & As05 & (52) \\
\hline HE 1005-1439 & 1.06 & 0.22 & $\ldots$ & $\ldots$ & $\ldots$ & $\ldots$ & $\ldots$ & $\ldots$ & $\ldots$ & $\ldots$ & $\mathrm{s}$ & As05 & (4) \\
\hline HE 1012-1540 & -0.22 & 0.02 & $\ldots$ & $\ldots$ & $\ldots$ & $\ldots$ & $<1.92$ & $\ldots$ & $<3.10$ & $\ldots$ & no & An89 & (11) \\
\hline HE 1031-0020 & 1.28 & 0.24 & 1.15 & 0.15 & 1.47 & $\ldots$ & $<0.94$ & $\ldots$ & 2.83 & $\ldots$ & $\mathrm{s}$ & An89 & (2) \\
\hline HE $1105+0027$ & 2.45 & 0.23 & 2.10 & 0.24 & $\ldots$ & $\ldots$ & 1.81 & 0.24 & $\ldots$ & $\ldots$ & rs & Gr98 & (5) \\
\hline HE $1135+0139$ & 1.13 & 0.24 & 0.93 & 0.25 & 1.17 & 0.27 & 0.33 & 0.25 & $\ldots$ & $\ldots$ & $\mathrm{s}$ & Gr98 & (5) \\
\hline HE $1135-0344$ & & $\ldots$ & $\ldots$ & $\ldots$ & $\ldots$ & $\ldots$ & $\ldots$ & $\ldots$ & $\ldots$ & $\ldots$ & no & Gr98 & (5) \\
\hline HE $1150-0428$ & -0.54 & 0.14 & $<1.15$ & $\ldots$ & $\ldots$ & $\ldots$ & $<1.52$ & $\ldots$ & $\ldots$ & $\ldots$ & no & An89 & (2) \\
\hline HE $1152-0355$ & 1.58 & $\ldots$ & 1.57 & $\ldots$ & $\ldots$ & $\ldots$ & $\ldots$ & $\ldots$ & $\ldots$ & $\ldots$ & $\mathrm{s}$ & As05 & (12) \\
\hline HE 1157-0518 & 2.14 & 0.24 & $\ldots$ & $\ldots$ & $\ldots$ & $\ldots$ & $\ldots$ & $\ldots$ & $\ldots$ & $\ldots$ & rs & As05 & (4) \\
\hline HE 1221-1948 & $\ldots$ & $\ldots$ & $\ldots$ & $\ldots$ & $\ldots$ & $\ldots$ & $\ldots$ & $\ldots$ & $\ldots$ & $\ldots$ & no & Gr98 & (5) \\
\hline HE 1249-3121 & $\ldots$ & $\ldots$ & $\ldots$ & $\ldots$ & $\ldots$ & $\ldots$ & $\ldots$ & $\ldots$ & $\ldots$ & $\ldots$ & no & Gr98 & (5) \\
\hline \multirow[t]{3}{*}{ HE $1300+0157$} & $<-0.85$ & $\ldots$ & $\ldots$ & $\ldots$ & $\ldots$ & $\ldots$ & $<1.56$ & $\ldots$ & $<2.78$ & $\ldots$ & no & As05 & (13) \\
\hline & & $\ldots$ & $\ldots$ & $\cdots$ & $\ldots$ & $\ldots$ & $\ldots$ & $\ldots$ & $\ldots$ & $\ldots$ & no & Gr98 & (5) \\
\hline & $<-0.56$ & & $\ldots$ & $\ldots$ & $\ldots$ & $\ldots$ & $\ldots$ & $\ldots$ & $\ldots$ & $\ldots$ & no & An89 & (11) \\
\hline HE 1300-0641 & -0.77 & 0.26 & $\ldots$ & $\ldots$ & $\ldots$ & $\ldots$ & $\ldots$ & $\ldots$ & $\ldots$ & $\ldots$ & no & Gr98 & (5) \\
\hline HE 1300-2201 & -0.04 & 0.27 & & $\ldots$ & $\ldots$ & $\ldots$ & $\ldots$ & $\ldots$ & $\ldots$ & $\ldots$ & no & Gr98 & (5) \\
\hline HE $1305+0007$ & 2.32 & $\ldots$ & 2.56 & $\ldots$ & 2.53 & $\ldots$ & 1.97 & $\ldots$ & 2.37 & $\ldots$ & rs & As05 & (12) \\
\hline HE 1305-0331 & $\cdots$ & $\ldots$ & $\ldots$ & $\ldots$ & $\ldots$ & $\ldots$ & $\ldots$ & $\ldots$ & $\ldots$ & $\ldots$ & no & Gr98 & (5) \\
\hline HE 1319-1935 & 1.89 & 0.26 & $\ldots$ & $\ldots$ & $\ldots$ & $\ldots$ & $\ldots$ & $\ldots$ & $\ldots$ & $\ldots$ & $\mathrm{s}$ & As05 & (4) \\
\hline HE 1327-2326 & $<1.58$ & 0.12 & $\ldots$ & $\ldots$ & $\ldots$ & $\ldots$ & $\ldots$ & $\ldots$ & $\ldots$ & $\ldots$ & no & As05 & $(14,15,16)$ \\
\hline HE 1327-2326 & $<1.58$ & 0.12 & $\ldots$ & $\ldots$ & $\ldots$ & $\ldots$ & $\ldots$ & $\ldots$ & $\ldots$ & $\ldots$ & no & As05 & $(14,15,16)$ \\
\hline HE 1330-0354 & -0.47 & 0.26 & $\ldots$ & $\ldots$ & $\ldots$ & $\ldots$ & $\ldots$ & $\ldots$ & $\ldots$ & $\ldots$ & no & Gr98 & (5) \\
\hline HE 1351-1049 & 0.13 & 0.25 & $\ldots$ & $\ldots$ & $\ldots$ & $\ldots$ & $\ldots$ & $\ldots$ & $\ldots$ & $\ldots$ & no & Gr98 & (5) \\
\hline HE 1410-0004 & 1.13 & 0.24 & $\ldots$ & $\ldots$ & $\ldots$ & $\ldots$ & $<2.47$ & $\ldots$ & $<3.34$ & $\ldots$ & $\mathrm{s}$ & An89 & (2) \\
\hline HE $1410+0213$ & 0.05 & 0.20 & -0.41 & 0.20 & 0.14 & 0.30 & $<0.60$ & $\ldots$ & $<1.52$ & $\ldots$ & no & As05 & (52) \\
\hline & 0.14 & $\cdots$ & $\ldots$ & ... & $\ldots$ & $\ldots$ & $\ldots$ & $\ldots$ & $\ldots$ & $\ldots$ & no & An89 & (2) \\
\hline HE 1413-1954 & & $\ldots$ & & & & $\ldots$ & & & $\ldots$ & $\ldots$ & no & Gr98 & (5) \\
\hline HE 1419-1324 & 0.88 & 0.10 & 0.82 & 0.10 & 0.83 & 0.10 & 0.53 & 0.10 & 2.15 & 0.10 & low-s & As05 & (52) \\
\hline HE 1429-0551 & 1.57 & 0.17 & $\ldots$ & $\ldots$ & $\ldots$ & $\ldots$ & $\ldots$ & $\ldots$ & $\ldots$ & $\ldots$ & $\mathrm{s}$ & As05 & (4) \\
\hline HE $1430-1123$ & 1.82 & 0.23 & $\ldots$ & $\ldots$ & $\ldots$ & $\ldots$ & $\ldots$ & $\ldots$ & $\ldots$ & $\ldots$ & $\mathrm{s}$ & Gr98 & (5) \\
\hline HE $1434-1442$ & 1.30 & 0.17 & $\ldots$ & $\ldots$ & $\ldots$ & $\ldots$ & $\ldots$ & $\ldots$ & 2.35 & $\ldots$ & $\mathrm{s}$ & An89 & (2) \\
\hline HE $1443+0113$ & 1.47 & 0.04 & $\ldots$ & $\ldots$ & $\ldots$ & $\ldots$ & $\ldots$ & $\ldots$ & $\ldots$ & $\ldots$ & $\mathrm{s}$ & An89 & (2) \\
\hline HE $1447+0102$ & 2.70 & 0.14 & $\ldots$ & $\ldots$ & $\ldots$ & $\ldots$ & $\ldots$ & $\ldots$ & $\ldots$ & $\ldots$ & rs & $\mathrm{As} 05$ & (4) \\
\hline HE 1509-0806 & 2.00 & 0.18 & 1.66 & 0.16 & 1.96 & 0.38 & $<1.00$ & $\ldots$ & 2.78 & $\ldots$ & $\mathrm{s}$ & An89 & (2) \\
\hline HE 1523-1155 & 1.72 & 0.14 & $\ldots$ & $\ldots$ & $\ldots$ & $\ldots$ & $\ldots$ & $\ldots$ & $\ldots$ & $\ldots$ & $\mathrm{s}$ & As05 & (4) \\
\hline
\end{tabular}


T. Masseron et al.: A holistic approach to carbon-enhanced metal-poor stars

Table 2. continued.

\begin{tabular}{|c|c|c|c|c|c|c|c|c|c|c|c|c|c|}
\hline 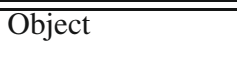 & $\begin{array}{c}\mathrm{Ba} / \mathrm{Fe}] \\
(16)\end{array}$ & $\begin{array}{c}\sigma \\
(17)\end{array}$ & $\begin{array}{c}{[\mathrm{La} / \mathrm{Fe}]} \\
(18)\end{array}$ & $\begin{array}{c}\sigma \\
(19)\end{array}$ & $\begin{array}{c}\overline{[\mathrm{Ce} / \mathrm{Fe}]} \\
(20)\end{array}$ & $\begin{array}{c}\sigma \\
(21)\end{array}$ & $\begin{array}{c}\overline{[\mathrm{Eu} / \mathrm{Fe}]} \\
(22)\end{array}$ & $\begin{array}{c}\sigma \\
(23)\end{array}$ & $\begin{array}{c}\mathrm{Pb} / \mathrm{Fe}] \\
(24)\end{array}$ & $\begin{array}{c}\sigma \\
(25)\end{array}$ & $\begin{array}{c}\text { Class } \\
(26)\end{array}$ & $\begin{array}{c}\text { Solar ref } \\
(27)\end{array}$ & $\begin{array}{l}\text { References } \\
(28)\end{array}$ \\
\hline HE 1528-0409 & 2.30 & 0.14 & $\ldots$ & $\ldots$ & $\ldots$ & $\ldots$ & $\ldots$ & $\ldots$ & $\ldots$ & $\ldots$ & rs & As05 & (4) \\
\hline HE 2148-1247 & 2.41 & 0.15 & 2.30 & 0.20 & 2.30 & 0.20 & 2.03 & 0.10 & 3.22 & 0.20 & rs & Gr98 & (17) \\
\hline HE $2150-0825$ & 1.70 & 0.22 & 1.41 & 0.25 & 1.48 & 0.28 & $\ldots$ & $\ldots$ & $\ldots$ & $\ldots$ & $\mathrm{s}$ & Gr98 & (5) \\
\hline \multirow[t]{2}{*}{ HE 2158-0348 } & 1.60 & $\ldots$ & 1.87 & 0.10 & 2.46 & 0.41 & 1.37 & 0.20 & 3.42 & 0.20 & rs & An89 & (6) \\
\hline & 1.66 & 0.15 & 1.54 & 0.21 & 1.96 & 0.21 & 0.87 & 0.15 & 2.77 & $\ldots$ & $\mathrm{s}$ & An89 & (2) \\
\hline HE 2221-0453 & 1.75 & 0.24 & & & $\ldots$ & $\ldots$ & $\ldots$ & $\ldots$ & $\ldots$ & $\ldots$ & $\mathrm{s}$ & As05 & (4) \\
\hline HE 2227-4044 & 1.38 & 0.24 & 1.28 & 0.26 & $\ldots$ & $\ldots$ & $\ldots$ & $\ldots$ & $\ldots$ & $\ldots$ & $\mathrm{s}$ & Gr98 & (5) \\
\hline HE 2228-0706 & 2.50 & 0.16 & $\ldots$ & $\ldots$ & $\ldots$ & $\ldots$ & $\ldots$ & $\ldots$ & $\ldots$ & $\ldots$ & rs & As05 & (4) \\
\hline \multirow{2}{*}{ HE 2232-0603 } & 1.57 & $\ldots$ & 1.22 & 0.05 & 0.83 & 0.07 & 0.67 & 0.10 & 2.12 & 0.20 & $\mathrm{~s}$ & An89 & (6) \\
\hline & 1.48 & 0.19 & 1.22 & 0.27 & 1.52 & $\ldots$ & $\ldots$ & $\ldots$ & 1.72 & $\ldots$ & $\mathrm{s}$ & An89 & (2) \\
\hline HE 2240-0412 & 1.37 & 0.23 & $\ldots$ & $\ldots$ & $\ldots$ & $\ldots$ & $\ldots$ & $\ldots$ & $\ldots$ & $\ldots$ & $\mathrm{s}$ & Gr98 & (5) \\
\hline HE 2330-0555 & 1.22 & 0.25 & $\ldots$ & $\ldots$ & $\ldots$ & $\ldots$ & $\ldots$ & $\ldots$ & $\ldots$ & $\ldots$ & $\mathrm{s}$ & As05 & (4) \\
\hline CS 22183-015 & 2.09 & 0.21 & 1.59 & 0.09 & 1.55 & 0.09 & 1.39 & 0.11 & 3.17 & 0.07 & rs & Gr96 & (18) \\
\hline \multirow[t]{3}{*}{ or HE 0058-0244 } & 1.89 & 0.12 & 1.53 & 0.11 & $\ldots$ & $\ldots$ & 1.37 & 0.16 & 3.00 & 0.07 & rs & Gr98 & (19) \\
\hline & 2.04 & 0.16 & 1.70 & 0.09 & 1.88 & 0.22 & 1.70 & 0.14 & 2.89 & $\ldots$ & rs & An89 & (2) \\
\hline & 1.74 & & 1.87 & 0.10 & 2.01 & $\ldots$ & 1.57 & 0.20 & 3.42 & 0.20 & rs & An89 & (6) \\
\hline CS 22877-001 & -0.49 & 0.17 & $\ldots$ & $\ldots$ & $\ldots$ & $\ldots$ & $<0.60$ & $\cdots$ & $\ldots$ & 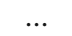 & no & Gr96 & (20) \\
\hline \multirow[t]{2}{*}{ CS 22880-074 } & 1.31 & 0.16 & 1.07 & 0.18 & 1.22 & 0.14 & 0.50 & 0.17 & 1.90 & 0.19 & $\mathrm{~s}$ & Gr96 & $(21,22)$ \\
\hline & 1.34 & $\ldots$ & 1.24 & $\ldots$ & $\ldots$ & $\ldots$ & 0.55 & $\ldots$ & $\ldots$ & $\ldots$ & $\mathrm{s}$ & An89 & (23) \\
\hline CS 22881-036 & 1.93 & $\ldots$ & 1.59 & $\ldots$ & $\ldots$ & $\ldots$ & 1.00 & $\ldots$ & $\ldots$ & $\ldots$ & $\mathrm{s}$ & An89 & (23) \\
\hline CS 22887-048 & 2.00 & 0.14 & 1.73 & 0.08 & $\ldots$ & $\ldots$ & 1.49 & 0.14 & 3.40 & 0.07 & rs & Gr98 & (19) \\
\hline CS 22891-171 & 2.48 & 0.20 & 2.12 & 0.05 & 2.06 & 0.18 & 1.73 & 0.10 & 1.85 & 0.20 & rs & As05 & (52) \\
\hline \multirow[t]{3}{*}{ CS 22892-052 } & 0.96 & 0.05 & 1.09 & 0.05 & 1.03 & 0.07 & 1.66 & 0.05 & 1.35 & 0.15 & rII & Gr98 & (24) \\
\hline & 1.01 & 0.07 & 1.11 & 0.06 & 1.02 & 0.12 & 1.49 & 0.06 & $\ldots$ & $\ldots$ & $\mathrm{r}$ & Gr98 & $(25,26,27,29)$ \\
\hline & 1.19 & 0.23 & 1.02 & 0.24 & $\ldots$ & $\ldots$ & 1.54 & 0.24 & $\ldots$ & $\ldots$ & $\mathrm{r}$ & Gr98 & (5) \\
\hline \multirow[t]{3}{*}{ CS 22898-027 } & 2.23 & 0.14 & 2.13 & 0.18 & 2.13 & 0.13 & 1.88 & 0.15 & 2.84 & 0.19 & rs & Gr96 & $(21,22)$ \\
\hline & 2.26 & 0.16 & 2.19 & 0.14 & $\ldots$ & $\ldots$ & 1.91 & 0.11 & 2.89 & 0.07 & rs & Gr98 & (19) \\
\hline & 2.27 & $\ldots$ & 2.28 & $\ldots$ & $\ldots$ & $\ldots$ & 1.94 & $\ldots$ & $\ldots$ & $\ldots$ & rs & An89 & (23) \\
\hline \multirow[t]{3}{*}{ CS 22942-019 } & 1.76 & 0.10 & 1.50 & 0.10 & 1.75 & 0.25 & 0.66 & 0.05 & $<2.03$ & $\ldots$ & $\mathrm{s}$ & As05 & (52) \\
\hline & 1.92 & 0.19 & 1.20 & 0.26 & 1.54 & 0.12 & 0.79 & 0.12 & $<1.60$ & $\ldots$ & $\mathrm{s}$ & Gr96 & $(21,22)$ \\
\hline & 1.50 & $\ldots$ & 1.85 & $\ldots$ & $\ldots$ & $\ldots$ & 0.80 & $\ldots$ & $\ldots$ & $\ldots$ & $\mathrm{s}$ & An89 & (23) \\
\hline CS 22945-017 & 0.55 & 0.20 & 0.99 & 0.20 & $<1.24$ & $\ldots$ & $<1.20$ & $\ldots$ & $<2.22$ & $\ldots$ & no & As05 & (52) \\
\hline \multirow[t]{4}{*}{ CS 22948-027 } & 2.26 & $\ldots$ & 2.32 & $\ldots$ & 2.20 & $\ldots$ & 1.88 & $\ldots$ & 2.72 & $\ldots$ & rs & Gr98 & (28) \\
\hline & 1.67 & $\ldots$ & & $\ldots$ & $\cdots$ & & $\ldots$ & $\ldots$ & $\ldots$ & $\ldots$ & rs & An89 & (23) \\
\hline & 1.85 & 0.14 & 1.91 & 0.15 & 1.99 & 0.14 & 1.57 & $\ldots$ & $\ldots$ & $\ldots$ & rs & Gr96 & (20) \\
\hline & 2.31 & 0.15 & $\ldots$ & $\ldots$ & $\ldots$ & $\ldots$ & $\ldots$ & $\ldots$ & $\ldots$ & $\ldots$ & $\mathrm{s}$ & As05 & (4) \\
\hline CS 22949-037 & -0.58 & 0.10 & $\ldots$ & $\ldots$ & $\ldots$ & $\ldots$ & $<0.04$ & $\ldots$ & $\ldots$ & $\ldots$ & no & Gr98 & (30) \\
\hline or HE 2323-0256 & -0.59 & 0.23 & $\ldots$ & $\ldots$ & $\ldots$ & $\ldots$ & $<0.80$ & $\ldots$ & $\ldots$ & $\ldots$ & no & An89 & (11) \\
\hline \multirow[t]{3}{*}{ CS 22956-028 } & 0.16 & 0.20 & $<0.50$ & $\ldots$ & 0.85 & 0.30 & $<0.91$ & $\ldots$ & $<1.33$ & $\ldots$ & no & As05 & $(52)$ \\
\hline & 0.56 & 0.15 & 0.67 & 0.20 & $\ldots$ & $\ldots$ & $<0.77$ & 0.10 & 1.92 & 0.20 & no & An89 & (6) \\
\hline & 0.37 & 0.26 & $\ldots$ & $\ldots$ & $\ldots$ & $\ldots$ & $\ldots$ & ․ & $\ldots$ & $\ldots$ & no & An89 & (31) \\
\hline CS 22957-027 & -0.95 & 0.12 & $\ldots$ & $\ldots$ & $\ldots$ & $\ldots$ & $<0.85$ & 0.21 & $\ldots$ & $\ldots$ & no & An89 & (32) \\
\hline or HE 2356-0410 & -0.93 & 0.14 & $\ldots$ & $\ldots$ & $\ldots$ & $\ldots$ & $\ldots$ & $\ldots$ & $\ldots$ & $\ldots$ & no & An89 & (33) \\
\hline & -2.00 & & $\ldots$ & $\ldots$ & $\ldots$ & $\ldots$ & $\ldots$ & $\ldots$ & $\ldots$ & $\ldots$ & no & An89 & (23) \\
\hline & -1.23 & 0.21 & $\ldots$ & $\ldots$ & $\ldots$ & $\ldots$ & $\ldots$ & $\ldots$ & $\ldots$ & ... & no & Gr96 & (34) \\
\hline & -0.71 & 0.25 & $<0.59$ & $\ldots$ & $\ldots$ & $\ldots$ & $<1.04$ & $\ldots$ & $\ldots$ & $\ldots$ & no & An89 & (2) \\
\hline & -0.91 & $\ldots$ & 0.47 & 0.20 & $\ldots$ & $\ldots$ & -0.53 & 0.20 & $<1.42$ & 0.20 & $\mathrm{r}$ & An89 & (6) \\
\hline CS 22958-042 & $<-0.53$ & 0.16 & $\ldots$ & $\ldots$ & $\ldots$ & $\ldots$ & $\ldots$ & $\ldots$ & $\ldots$ & $\ldots$ & no & As05 & (35) \\
\hline CS 22960-053 & 0.86 & 0.14 & $\ldots$ & $\ldots$ & $\ldots$ & $\ldots$ & $\ldots$ & $\ldots$ & $\ldots$ & $\ldots$ & no & As05 & (4) \\
\hline CS 22967-007 & 2.09 & 0.12 & 1.47 & 0.10 & 1.69 & 0.05 & 0.77 & 0.20 & 2.82 & 0.10 & $\mathrm{~s}$ & An89 & (6) \\
\hline CS 29495-042 & 1.83 & 0.18 & 1.27 & 0.20 & 1.48 & 0.05 & 0.77 & 0.20 & 1.32 & 0.20 & $\mathrm{~s}$ & An89 & (6) \\
\hline CS 29497-030 & 2.17 & 0.15 & 2.10 & 0.10 & 2.14 & 0.17 & 1.44 & 0.15 & 3.55 & $\ldots$ & rs & Gr98 & $(36)$ \\
\hline & 2.32 & 0.11 & 2.22 & 0.10 & 2.10 & 0.10 & 1.99 & 0.10 & 3.65 & 0.13 & rs & An89 & (37) \\
\hline CS 29497-034 & 2.03 & $\ldots$ & 2.12 & $\ldots$ & 1.95 & $\ldots$ & 1.80 & $\ldots$ & 2.95 & $\ldots$ & rs & Gr98 & (28) \\
\hline & 1.79 & 0.10 & 1.87 & 0.20 & $\ldots$ & $\ldots$ & $<1.57$ & 0.10 & 4.52 & 0.20 & $\mathrm{~s}$ & An89 & (6) \\
\hline or HE 0039-2635 & 2.23 & 0.13 & $\ldots$ & $\ldots$ & $\ldots$ & $\ldots$ & $\ldots$ & $\ldots$ & $\ldots$ & $\ldots$ & rs & As05 & (4) \\
\hline CS 29498-043 & -0.46 & 0.20 & $\ldots$ & $\ldots$ & $\ldots$ & $\ldots$ & $\ldots$ & $\ldots$ & $\ldots$ & $\ldots$ & no & Gr96 & (38) \\
\hline & -0.45 & 0.20 & $\ldots$ & $\ldots$ & $\ldots$ & $\ldots$ & $\ldots$ & $\ldots$ & $\ldots$ & $\ldots$ & no & Gr96 & (34) \\
\hline CS 29502-092 & -0.82 & 0.27 & $\ldots$ & $\ldots$ & $\ldots$ & $\ldots$ & $<0.40$ & $\ldots$ & $\ldots$ & $\ldots$ & no & Gr96 & (20) \\
\hline & -1.37 & 0.07 & $<0.06$ & $\ldots$ & $\ldots$ & $\ldots$ & $<0.21$ & $\ldots$ & $<1.53$ & $\ldots$ & no & Gr98 & (19) \\
\hline CS 29503-010 & 1.50 & 0.16 & $\ldots$ & $\ldots$ & $\ldots$ & $\ldots$ & $\ldots$ & $\ldots$ & $\ldots$ & $\ldots$ & $\mathrm{s}$ & As05 & (4) \\
\hline CS 29509-027 & 1.33 & 0.13 & $\ldots$ & $\ldots$ & $\ldots$ & $\ldots$ & $\ldots$ & $\ldots$ & $\ldots$ & $\ldots$ & $\mathrm{s}$ & An89 & (31) \\
\hline CS 29526-110 & 2.11 & 0.17 & 1.69 & 0.24 & 2.01 & 0.22 & 1.73 & 0.20 & 3.30 & 0.24 & rs & Gr96 & $(21,22)$ \\
\hline CS 29528-028 & 3.27 & 0.20 & $\ldots$ & $\ldots$ & $\ldots$ & $\ldots$ & $\ldots$ & $\ldots$ & $\ldots$ & $\ldots$ & rs & As05 & (4) \\
\hline CS 29528-041 & 0.97 & 0.10 & $\ldots$ & $\ldots$ & $\ldots$ & $\ldots$ & $\ldots$ & $\ldots$ & $\ldots$ & $\ldots$ & no & As05 & (35) \\
\hline CS 30301-015 & 1.45 & 0.16 & 0.84 & 0.25 & 1.16 & 0.15 & 0.20 & 0.18 & 1.70 & 0.24 & $\mathrm{~s}$ & Gr96 & $(21,22)$ \\
\hline
\end{tabular}


Table 2. continued.

\begin{tabular}{|c|c|c|c|c|c|c|c|c|c|c|c|c|c|}
\hline Object & $\begin{array}{c}\mathrm{Ba} / \mathrm{Fe}] \\
(16)\end{array}$ & $\begin{array}{c}\sigma \\
(17)\end{array}$ & $\begin{array}{c}\mathrm{La} / \mathrm{Fe}] \\
(18)\end{array}$ & $\begin{array}{c}\sigma \\
(19)\end{array}$ & $\begin{array}{c}\mathrm{Ce} / \mathrm{Fe}] \\
(20)\end{array}$ & $\begin{array}{c}\sigma \\
(21)\end{array}$ & $\begin{array}{c}{[\mathrm{Eu} / \mathrm{Fe}]} \\
(22)\end{array}$ & $\begin{array}{c}\sigma \\
(23)\end{array}$ & $\begin{array}{c}\mathrm{Pb} / \mathrm{Fe}] \\
(24)\end{array}$ & $\begin{array}{c}\sigma \\
(25)\end{array}$ & $\begin{array}{c}\text { Class } \\
(26)\end{array}$ & $\begin{array}{c}\text { Solar ref } \\
(27)\end{array}$ & $\begin{array}{l}\text { References } \\
(28)\end{array}$ \\
\hline CS 30314-067 & -0.57 & 0.14 & & & & & $<-0.50$ & & & & no & Gr96 & $(20)$ \\
\hline CS 30315-091 & 1.62 & 0.15 & 0.87 & 0.20 & 1.41 & 0.12 & 0.37 & 0.20 & 1.92 & 0.30 & $\mathrm{~s}$ & An89 & (6) \\
\hline \multirow[t]{2}{*}{ CS 30322-023 } & 0.52 & 0.05 & 0.46 & 0.05 & 0.59 & 0.24 & -0.63 & 0.10 & 1.49 & 0.20 & low-s & As05 & $(52)$ \\
\hline & 0.59 & 0.17 &.. & & $\cdots$ &.. & $\cdots$ &. & & & no & As05 & (4) \\
\hline CS 30323-107 & 1.90 & 0.17 & 1.12 & 0.10 & 1.46 & 0.06 & $<0.57$ & 0.10 & 2.52 & 0.20 & $\mathrm{~s}$ & An89 & (6) \\
\hline CS 30338-089 & 2.22 & 0.15 & 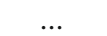 & $\ldots$ & . & $\ldots$ & $\ldots$ & & 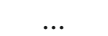 & $\ldots$ & rs & As05 & (4) \\
\hline CS 30338-089 & 1.87 & 0.38 & 1.57 & 0.20 & 3.09 & 0.26 & 1.77 & 0.20 & 3.72 & 0.20 & rs & An89 & (6) \\
\hline \multirow[t]{2}{*}{ CS 31062-050 } & 2.30 & 0.15 & 2.44 & 0.20 & 2.10 & 0.12 & 1.84 & 0.13 & 2.90 & 0.24 & rs & Gr96 & $(21,22)$ \\
\hline & 2.80 & 0.20 & 2.12 & 0.12 & 2.02 & 0.16 & 1.79 & 0.07 & 2.81 & 0.15 & rs & Gr96 & (39) \\
\hline CS 31080-095 & 0.77 & 0.15 & & & $\cdots$ & $\ldots$ & & & & & no & As05 & (35) \\
\hline BS $16080-175$ & 1.55 & 0.07 & 1.65 & 0.07 & $\ldots$ & $\ldots$ & 1.05 & 0.07 & 2.60 & 0.07 & rs & Gr98 & (19) \\
\hline BS 16929-005 & -0.48 & 0.12 & $\cdots$ & $\cdots$ & $\ldots$ & $\ldots$ & $\ldots$ & $\ldots$ & $\ldots$ & $\ldots$ & no & As05 & (4) \\
\hline BS 17436-058 & 1.61 & 0.14 & 1.49 & 0.17 & $\ldots$ & $\ldots$ & 1.17 & 0.11 & 2.26 & 0.07 & rs & Gr98 & (19) \\
\hline HD 26 & 1.85 & 0.20 & 1.39 & 0.10 & 1.67 & 0.10 & 0.75 & 0.10 & 2.02 & 0.10 & $\mathrm{~s}$ & As05 & $(52)$ \\
\hline HD 5223 & 1.82 & $\ldots$ & 1.76 & $\ldots$ & 1.87 & $\ldots$ & ... & $\ldots$ & $<2.21$ & $\ldots$ & $\mathrm{s}$ & As05 & (12) \\
\hline HD 187861 & 1.39 & 0.10 & 1.73 & 0.20 & 1.37 & 0.14 & 1.34 & 0.20 & 2.86 & 0.20 & rs & As05 & $(52)$ \\
\hline \multirow[t]{3}{*}{ HD 196944} & 1.22 & 0.20 & 0.86 & 0.05 & 0.99 & 0.12 & 0.22 & 0.20 & 1.99 & 0.10 & $\mathrm{~s}$ & As05 & $(52)$ \\
\hline & 1.10 & 0.19 & 0.91 & 0.28 & 1.01 & 0.19 & 0.17 & 0.19 & 1.90 & 0.24 & $\mathrm{~s}$ & Gr96 & $(21,22)$ \\
\hline & 1.26 & 0.30 & & $\cdots$ & 0.61 & $\cdots$ & $\cdots$ & & $\ldots$ & $\ldots$ & $\mathrm{s}$ & An89 & $(40)$ \\
\hline HD 206983 & 0.92 & 0.20 & 0.86 & 0.05 & 1.06 & 0.10 & 0.57 & 0.10 & 1.49 & 0.20 & $\mathrm{~s}$ & As05 & $(52)$ \\
\hline HD 224959 & 2.19 & 0.20 & 2.03 & 0.05 & 1.91 & 0.09 & 1.74 & 0.10 & 3.06 & 0.10 & rs & As05 & $(52)$ \\
\hline HKIII7435-00532 & 0.86 & 0.29 & 0.78 & 0.21 & 1.17 & 0.20 & 0.48 & 0.20 & $\ldots$ & $\ldots$ & low-s & Gr98 & (51) \\
\hline \multirow[t]{2}{*}{ LP 625-44 } & 2.74 & 0.20 & 2.46 & 0.13 & 2.27 & 0.12 & 1.97 & 0.20 & 2.55 & 0.20 & rs & Gr96 & (41) \\
\hline & 2.81 & 0.24 & 2.40 & 0.26 & 2.22 & 0.23 & 1.72 & 0.24 & 2.60 & 0.22 & rs & Gr96 & (42) \\
\hline LP 706-7 & 2.01 & 0.14 & 1.81 & 0.19 & 1.86 & 0.31 & 1.40 & 0.20 & 2.28 & 0.20 & rs & Gr96 & (41) \\
\hline or CS 31062-012 & 1.98 & 0.16 & 2.02 & 0.18 & 2.12 & 0.13 & 1.62 & 0.14 & 2.40 & 0.19 & rs & Gr96 & $(21,22)$ \\
\hline G 77-61 & $<1.00$ & $\ldots$ & & & $\ldots$ & $\ldots$ & $<3.00$ & & $\ldots$ & $\ldots$ & no & Gr98 & (43) \\
\hline V 543Oph & 0.34 & $\ldots$ & 0.37 & 0.09 & $\ldots$ & $\ldots$ & 0.65 & 0.20 & $\ldots$ & $\ldots$ & no & Gr98 & (44) \\
\hline
\end{tabular}


Table 3. Same as Table 1 for stars with $[\mathrm{C} / \mathrm{Fe}]<0.9$.

\begin{tabular}{|c|c|c|c|c|c|c|c|c|c|c|c|c|c|c|}
\hline $\begin{array}{l}\text { Object } \\
\text { (1) }\end{array}$ & $\begin{array}{c}T_{\text {eff }} \log g \\
\text { (2) }\end{array}$ & $\begin{array}{c}{[\mathrm{Fe} / \mathrm{H}]} \\
(3)\end{array}$ & $\begin{array}{c}\sigma \\
(4)\end{array}$ & $\begin{array}{c}{[\mathrm{C} / \mathrm{Fe}]} \\
(5)\end{array}$ & $\begin{array}{c}\sigma \\
(6)\end{array}$ & $\begin{array}{c}{ }^{12} C /{ }^{13} C \\
\text { (7) }\end{array}$ & $\begin{array}{c}\sigma \\
(8)\end{array}$ & $\begin{array}{c}{[\mathrm{N} / \mathrm{Fe}]} \\
(9)\end{array}$ & $\begin{array}{c}\sigma \\
(10)\end{array}$ & $\begin{array}{c}\mathrm{O} / \mathrm{Fe}] \\
(11)\end{array}$ & $\begin{array}{c}\sigma \\
(12)\end{array}$ & $\begin{array}{c}\text { Non-LTE } \\
\text { (13) }\end{array}$ & $\begin{array}{c}{[\mathrm{Mg} / \mathrm{Fe}]} \\
(14)\end{array}$ & $\begin{array}{c}\sigma \\
(15)\end{array}$ \\
\hline \multicolumn{15}{|c|}{ Ba stars } \\
\hline $\mathrm{BD}+18: 5215$ & 63004.20 & -0.44 & 0.04 & 0.59 & 0.08 & $\ldots$ & $\ldots$ & $\ldots$ & 0.13 & 0.43 & 0.10 & $\ldots$ & 0.17 & 0.05 \\
\hline HR 107 & 66504.00 & -0.34 & 0.04 & 0.23 & 0.08 & $\ldots$ & $\ldots$ & $\ldots$ & 0.13 & -0.07 & 0.10 & $\ldots$ & 0.08 & 0.05 \\
\hline HD 749 & 45802.30 & -0.06 & 0.18 & 0.20 & 0.20 & $\ldots$ & $\ldots$ & -0.04 & 0.18 & 0.21 & 0.24 & $\ldots$ & -0.12 & 0.20 \\
\hline \multirow[t]{2}{*}{ HD 5424} & 46002.30 & -0.21 & 0.14 & 0.12 & 0.10 & 8 & 2 & 0.43 & 0.10 & 0.00 & 0.20 & $\ldots$ & 0.34 & 0.34 \\
\hline & 47001.80 & -0.51 & 0.18 & 0.39 & 0.20 & $\ldots$ & $\ldots$ & 0.41 & 0.18 & 0.18 & 0.24 & $\ldots$ & 0.20 & 0.20 \\
\hline HD 8270 & 60704.20 & -0.44 & 0.04 & 0.31 & 0.08 & $\ldots$ & $\ldots$ & 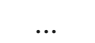 & 0.13 & 0.08 & 0.10 & 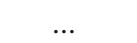 & -0.04 & 0.05 \\
\hline HD 12392 & 50003.20 & -0.06 & 0.18 & 0.40 & 0.20 & $\ldots$ & $\ldots$ & 0.57 & 0.18 & 0.18 & 0.24 & $\ldots$ & 0.05 & 0.20 \\
\hline HD 13551 & 60503.70 & -0.44 & 0.04 & 0.24 & 0.08 & $\ldots$ & $\ldots$ & $\ldots$ & 0.13 & $<0.43$ & 0.10 & $\ldots$ & 0.25 & 0.05 \\
\hline HD 22589 & 56303.30 & -0.12 & 0.04 & 0.30 & 0.08 & $\ldots$ & $\ldots$ & 0.19 & 0.13 & $<0.03$ & 0.10 & $\ldots$ & 0.21 & 0.05 \\
\hline HD 24035 & 45002.00 & -0.14 & 0.18 & 0.15 & 0.10 & 120 & 5 & 0.46 & 0.10 & $1-0.12$ & 0.20 & $\ldots$ & -0.24 & 0.14 \\
\hline HD 27271 & 48302.30 & -0.09 & 0.18 & 0.24 & 0.20 & $\ldots$ & $\ldots$ & 0.48 & 0.18 & 0.19 & 0.24 & $\ldots$ & -0.12 & 0.20 \\
\hline HD 48565 & 60503.80 & -0.71 & 0.04 & 0.37 & 0.08 & $\ldots$ & $\ldots$ & $\ldots$ & 0.13 & 0.03 & 0.10 & $\ldots$ & 0.02 & 0.05 \\
\hline HD 76225 & 63303.70 & -0.34 & 0.04 & 0.35 & 0.08 & $\ldots$ & $\ldots$ & $\ldots$ & 0.13 & 0.13 & 0.10 & $\ldots$ & -0.01 & 0.05 \\
\hline HD 87080 & 55503.70 & -0.49 & 0.04 & 0.33 & 0.08 & $\ldots$ & $\ldots$ & $\ldots$ & 0.13 & & 0.10 & $\ldots$ & 0.04 & 0.05 \\
\hline HD 89948 & 60104.20 & -0.28 & 0.04 & 0.25 & 0.08 & $\ldots$ & $\ldots$ & $\ldots$ & 0.13 & 0.28 & 0.10 & $\ldots$ & 0.07 & 0.05 \\
\hline HD 92545 & 62704.00 & -0.15 & 0.04 & 0.32 & 0.08 & $\ldots$ & $\ldots$ & $\ldots$ & 0.13 & 0.33 & 0.10 & $\ldots$ & -0.08 & 0.05 \\
\hline 6191 & 58904.20 & -0.22 & 0.04 & 0.40 & 0.08 & $\ldots$ & $\ldots$ & $\ldots$ & 0.13 & 0.38 & 0.10 & $\ldots$ & 0.07 & 0.05 \\
\hline HD & 64003.60 & -0.56 & 0.04 & 0.39 & 0.08 & $\ldots$ & $\ldots$ & $\ldots$ & 0.13 & $<0.33$ & 0.10 & $\ldots$ & 0.08 & 0.05 \\
\hline HD 116869 & 48502.10 & -0.35 & 0.18 & 0.11 & 0.20 & $\ldots$ & $\ldots$ & 0.26 & 0.18 & -0.04 & 0.24 & $\ldots$ & -0.04 & 0.20 \\
\hline HD 123396 & 44801.20 & -1.19 & 0.18 & 0.50 & 0.20 & $\ldots$ & $\ldots$ & -0.05 & 0.18 & 0.42 & 0.24 & $\ldots$ & 0.46 & 0.20 \\
\hline HD 123585 & 64504.20 & -0.44 & 0.04 & 0.79 & 0.08 & $\ldots$ & $\ldots$ & $\ldots$ & 0.13 & 0.33 & 0.10 & $\ldots$ & 0.04 & 0.05 \\
\hline HD 147609 & 59603.30 & -0.45 & 0.04 & 0.51 & 0.08 & $\ldots$ & $\ldots$ & $\ldots$ & 0.13 & $\ldots$ & 0.10 & $\ldots$ & -0.04 & 0.05 \\
\hline HD 150862 & 63104.60 & -0.11 & 0.04 & 0.44 & 0.08 & $\ldots$ & $\ldots$ & $\ldots$ & 0.13 & 0.28 & 0.10 & . & -0.15 & 0.05 \\
\hline HD 1 & 52003.50 & 0.02 & 0.12 & 0.09 & 0.10 & 40 & 10 & -0.00 & 0.10 & -0.18 & 0.20 & . & -0.22 & 0.11 \\
\hline HD 188985 & 61904.30 & -0.25 & 0.04 & 0.39 & 0.08 & $\ldots$ & $\ldots$ & 0.87 & 0.13 & 0.44 & 0.10 & $\ldots$ & -0.01 & 0.05 \\
\hline HD 207585 & 58004.00 & -0.20 & 0.19 & 0.51 & 0.10 & 50 & 10 & 0.12 & 0.10 & 0.14 & 0.20 & $\ldots$ & -0.03 & 0.28 \\
\hline HD 210709 & 46802.30 & -0.07 & 0.18 & -0.02 & 0.20 & $\ldots$ & $\ldots$ & 0.23 & 0.18 & -0.02 & 0.24 & $\ldots$ & 0.04 & 0.20 \\
\hline HD 210910 & 47702.00 & -0.37 & 0.18 & 0.30 & 0.20 & $\ldots$ & $\ldots$ & $\ldots$ & 0.18 & 0.44 & 0.24 & $\ldots$ & 0.11 & 0.20 \\
\hline HD 2 & 48002.50 & -0.12 & 0.11 & -0.07 & 0.10 & 12 & 2 & 0.24 & 0.10 & -0.04 & 0.20 & $\ldots$ & -0.20 & 0.13 \\
\hline $\mathrm{HD} 2$ & 48001.80 & -0.34 & 0.21 & -0.15 & 0.10 & 8 & 2 & 0.26 & 0.10 & -0.12 & 0.20 & $\ldots$ & 0.12 & 0.27 \\
\hline HD & 61903.90 & -0.58 & 0.04 & 0.57 & 0.08 & $\ldots$ & $\ldots$ & 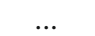 & 0.13 & 0.43 & 0.10 & $\ldots$ & 0.13 & 0.05 \\
\hline \multirow[t]{2}{*}{ HD 223938} & 51502.70 & -0.35 & 0.18 & 0.36 & 0.20 & $\ldots$ & $\ldots$ & 0.33 & 0.18 & 0.35 & 0.24 & $\ldots$ & 0.09 & 0.20 \\
\hline & \multicolumn{14}{|c|}{ rII stars } \\
\hline $\mathrm{CS} 2$ & 702.80 & -2.93 & 0.20 & 0.42 & & $\ldots$ & $\ldots$ & $\ldots$ & $\ldots$ & $\ldots$ & $\ldots$ & $\ldots$ & & \\
\hline $\mathrm{CS} 2$ & 32.45 & -2.81 & 0.13 & 0.05 & 0.17 & $\ldots$ & $\ldots$ & $\ldots$ & $\ldots$ & $\ldots$ & $\ldots$ & $\ldots$ & 0.28 & 0.15 \\
\hline CS & 50132.23 & -2.81 & 0.13 & 0.09 & 0.18 & $\ldots$ & $\ldots$ & $\ldots$ & $\ldots$ & & $\ldots$ & $\ldots$ & 0.31 & 0.15 \\
\hline 82-001 & 48251.5 & -2.90 & 0.13 & 0.20 & 0.05 & $>20$ & $\ldots$ & $\ldots$ & $\ldots$ & 0.59 & $\ldots$ & $\ldots$ & 0.36 & 0.13 \\
\hline HE 0430-4901 & 52963.12 & -2.72 & 0.12 & -0.04 & 0.18 & $\ldots$ & $\ldots$ & $\ldots$ & $\ldots$ & $\ldots$ & $\ldots$ & $\ldots$ & 0.17 & 0.16 \\
\hline HE 0432-0923 & 51312.64 & -3.19 & 0.13 & 0.11 & 0.17 & $\ldots$ & $\cdots$ & $\ldots$ & $\ldots$ & $\ldots$ & ... & $\ldots$ & 0.34 & 0.16 \\
\hline HE 1 & 52242.64 & -2.73 & 0.14 & 0.41 & 0.17 & $\ldots$ & $\ldots$ & $\ldots$ & $\ldots$ & $\ldots$ & $\ldots$ & $\ldots$ & 0.22 & 0.16 \\
\hline HE 1219-0312 & 51402.40 & -2.81 & 0.12 & -0.21 & 0.19 & $\ldots$ & $\ldots$ & $\ldots$ & $\ldots$ & $\ldots$ & $\ldots$ & $\ldots$ & 0.04 & 0.16 \\
\hline HE $2224+0143$ & 51982.66 & -2.58 & 0.12 & 0.22 & 0.17 & $\ldots$ & $\ldots$ & $\ldots$ & $\ldots$ & $\ldots$ & $\ldots$ & $\ldots$ & 0.32 & 0.15 \\
\hline HE 2327-5642 & 50482.22 & -2.95 & 0.12 & 0.30 & 0.19 & $\ldots$ & $\ldots$ & $\ldots$ & $\ldots$ & $\ldots$ & $\ldots$ & $\ldots$ & 0.14 & 0.15 \\
\hline \multicolumn{15}{|c|}{ rI stars } \\
\hline Object & $T_{\text {eff }} \log g$ & {$[\mathrm{Fe} / \mathrm{H}]$} & $\sigma$ & {$[\mathrm{C} / \mathrm{Fe}]$} & $\sigma$ & ${ }^{12} \mathrm{C} /{ }^{13} \mathrm{C}$ & $\sigma$ & {$[\mathrm{N} / \mathrm{Fe}]$} & $\sigma$ & {$[\mathrm{O} / \mathrm{Fe}]$} & $\sigma$ & Jon-LTE & {$[\mathrm{Mg} / \mathrm{Fe}]$} & $\sigma$ \\
\hline & (2) & (3) & (4) & & $(6)$ & (7) & $(8)$ & (9) & $(10)$ & $(11)$ & $(12)$ & (13) & $(14)$ & $(15)$ \\
\hline HD 122563 & 46001.1 & -2.82 & 0.17 & -0.47 & 0.05 & 5 & 2 & 0.70 & 0.15 & 0.56 & 0.12 & 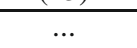 & 0.36 & 0.12 \\
\hline HD 2796 & 49501.5 & -2.47 & 0.13 & -0.40 & 0.06 & 4 & 2 & 0.85 & 0.08 & 0.44 & 0.05 & $\ldots$ & 0.25 & 0.14 \\
\hline HD 186478 & 47001.3 & -2.59 & 0.18 & -0.22 & 0.07 & 5 & 2 & 0.62 & 0.12 & 0.69 & 0.12 & $\ldots$ & 0.39 & 0.08 \\
\hline $\mathrm{BD}+17: 3248$ & 52501.4 & -2.07 & 0.15 & -0.33 & 0.05 & 10 & 5 & 0.65 & 0.10 & 0.63 & 0.05 & $\ldots$ & 0.19 & 0.10 \\
\hline BD -18:5550 & 47501.4 & -3.06 & 0.12 & -0.02 & 0.04 & $>40$ & $\ldots$ & -0.36 & 0.10 & 0.36 & 0.12 & $\ldots$ & 0.31 & 0.15 \\
\hline CD -38:245 & 48001.5 & -4.19 & 0.20 & -0.33 & $\ldots$ & $\ldots$ & $\ldots$ & 1.07 & 0.20 & $\ldots$ & $\ldots$ & $\ldots$ & 0.20 & 0.08 \\
\hline BS 16467-062 & 52002.5 & -3.77 & 0.14 & 0.25 & 0.12 & $\ldots$ & $\ldots$ & 0.85 & $\ldots$ & $\ldots$ & $\ldots$ & $\ldots$ & 0.16 & 0.09 \\
\hline BS 16477-003 & 49001.7 & -3.36 & 0.12 & 0.29 & 0.08 & $>30$ & $\ldots$ & 0.14 & $\ldots$ & $\ldots$ & $\ldots$ & $\ldots$ & 0.28 & 0.15 \\
\hline BS 17569-049 & 47001.2 & -2.88 & 0.17 & -0.05 & 0.05 & 6 & 2 & 0.86 & 0.12 & $\ldots$ & $\ldots$ & $\ldots$ & 0.25 & 0.20 \\
\hline CS 22169-035 & 47001.2 & -3.04 & 0.19 & -0.16 & 0.05 & 6 & 2 & 1.02 & 0.13 & $\ldots$ & $\cdots$ & $\ldots$ & 0.09 & 0.11 \\
\hline CS 22172-002 & 48001.3 & -3.86 & 0.17 & $\ldots$ & 0.10 & $>10$ & $\ldots$ & 0.24 & 0.20 & $<0.98$ & 0.12 & .. & 0.20 & 0.08 \\
\hline CS 22186-025 & 49001.5 & -3.00 & 0.14 & -0.54 & 0.10 & $\ldots$ & $\ldots$ & 0.98 & 0.08 & 0.53 & 0.05 & $\ldots$ & 0.36 & 0.14 \\
\hline CS 22189-009 & 49001.7 & -3.49 & 0.15 & 0.34 & 0.08 & 15 & 7 & 0.27 & 0.12 & $\ldots$ & $\ldots$ & $\ldots$ & 0.11 & 0.06 \\
\hline CS 22873-055 & 45500.7 & -2.99 & 0.14 & -0.63 & 0.06 & 4 & 2 & 1.07 & 0.20 & 0.46 & 0.12 & & 0.42 & 0.14 \\
\hline CS 22873-166 & 45500.9 & -2.97 & 0.19 & -0.13 & 0.08 & 5 & 2 & 1.05 & 0.20 & $\ldots$ & $\ldots$ & $\cdots$ & 0.52 & 0.18 \\
\hline CS 22878-101 & 48001.3 & -3.25 & 0.12 & -0.21 & 0.10 & 5 & 2 & 1.33 & 0.10 & $\ldots$ & $\ldots$ & $\ldots$ & 0.44 & 0.11 \\
\hline
\end{tabular}


Table 3. continued.

\begin{tabular}{|c|c|c|c|c|c|c|c|c|c|c|c|c|c|c|}
\hline $\begin{array}{l}\text { Object } \\
\text { (1) }\end{array}$ & $\begin{array}{c}T_{\text {eff }} \log g \\
\text { (2) }\end{array}$ & $\begin{array}{c}\mathrm{Fe} / \mathrm{H}] \\
\text { (3) }\end{array}$ & $\begin{array}{c}\sigma \\
(4)\end{array}$ & $\begin{array}{c}\overline{[\mathrm{C} / \mathrm{Fe}]} \\
(5)\end{array}$ & $\begin{array}{c}\sigma \\
(6)\end{array}$ & $\begin{array}{c}{ }^{12} C /{ }^{13} C \\
\text { (7) }\end{array}$ & $\begin{array}{c}\sigma \\
(8)\end{array}$ & $\begin{array}{c}{[\mathrm{N} / \mathrm{Fe}]} \\
(9)\end{array}$ & $\begin{array}{c}\sigma \\
(10)\end{array}$ & $\begin{array}{c}\mathrm{OO} / \mathrm{Fe}] \\
(11)\end{array}$ & $\begin{array}{c}\sigma \\
(12)\end{array}$ & $\begin{array}{c}\text { Non-LTE } \\
\text { (13) }\end{array}$ & $\begin{array}{c}\overline{[\mathrm{Mg} / \mathrm{Fe}]} \\
(14)\end{array}$ & $\begin{array}{c}\sigma \\
(15)\end{array}$ \\
\hline CS 22885-096 & 50502.6 & -3.78 & 0.17 & 0.26 & 0.06 & & & 0.26 & 0.13 & & & ; & 0.19 & 0.12 \\
\hline CS 22891-209 & 47001.0 & -3.29 & 0.14 & -0.57 & 0.05 & 5 & 2 & 1.12 & 0.10 & 0.71 & 0.12 & $\ldots$ & 0.34 & 0.14 \\
\hline CS 22896-154 & 52502.7 & -2.69 & 0.19 & 0.23 & 0.05 & $>40$ & .... & -0.23 & 0.12 & 0.88 & 0.05 & $\ldots$ & 0.08 & 0.12 \\
\hline CS 22897-008 & 49001.7 & -3.41 & 0.15 & 0.58 & 0.05 & 20 & 7 & 0.24 & 0.15 & $\ldots$ & & $\ldots$ & 0.26 & 0.12 \\
\hline CS 22948-066 & 51001.8 & -3.14 & 0.11 & 0.08 & 0.10 & $\ldots$ & $\ldots$ & 1.22 & 0.10 & 0.83 & 0.05 & $\ldots$ & 0.28 & 0.08 \\
\hline CS 22952-015 & 48001.3 & -3.43 & 0.14 & -0.59 & 0.08 & $\ldots$ & $\ldots$ & 1.31 & 0.10 & $\ldots$ & $\ldots$ & $\ldots$ & $\ldots$ & 0.08 \\
\hline \multirow[t]{2}{*}{ CS 22953-003 } & 50002.00 & -2.88 & 0.16 & 0.39 & 0.10 & 20 & 5 & 0.70 & 0.10 & $<1.22$ & . & $\ldots$ & 0.46 & 0.19 \\
\hline & 51002.3 & -2.84 & 0.15 & 0.32 & 0.03 & 20 & 7 & 0.12 & 0.10 & 0.69 & 0.05 & $\ldots$ & 0.13 & 0.09 \\
\hline CS 22956-050 & 49001.7 & -3.33 & 0.13 & 0.27 & 0.05 & $\ldots$ & $\ldots$ & 0.31 & 0.10 & 1.06 & 0.05 & $\ldots$ & 0.37 & 0.11 \\
\hline CS 22966-057 & 53002.2 & -2.62 & 0.12 & 0.06 & 0.05 & $\ldots$ & $\ldots$ & 0.10 & 0.12 & 0.99 & 0.05 & $\ldots$ & 0.12 & 0.20 \\
\hline CS 22968-014 & 48501.7 & -3.56 & 0.15 & 0.26 & 0.06 & 30 & 7 & 0.24 & 0.10 & 0.84 & 0.12 & $\ldots$ & 0.19 & 0.13 \\
\hline CS 29491-053 & 47001.3 & -3.04 & 0.16 & -0.21 & 0.05 & 7 & 2 & 0.82 & 0.15 & 0.70 & 0.12 & $\ldots$ & 0.53 & 0.16 \\
\hline CS 29495-041 & 48001.5 & -2.82 & 0.15 & -0.04 & 0.06 & 14 & 7 & 0.40 & 0.10 & 0.62 & 0.12 & $\ldots$ & 0.33 & 0.13 \\
\hline CS 29502-042 & 51002.5 & -3.19 & 0.11 & 0.16 & 0.04 & $>30$ & $\ldots$ & -0.43 & 0.20 & $\ldots$ & $\cdots$ & $\ldots$ & 0.23 & 0.10 \\
\hline CS 29516-024 & 46501.2 & -3.06 & 0.10 & -0.04 & 0.05 & 20 & 7 & -0.76 & 0.20 & 0.56 & 0.12 & $\ldots$ & 0.48 & 0.11 \\
\hline CS 29518-051 & 52002.6 & -2.69 & 0.13 & -0.08 & 0.05 & 8 & 2 & 0.82 & 0.15 & 0.74 & 0.05 & $\ldots$ & 0.20 & 0.11 \\
\hline CS 30325-094 & 49502.0 & -3.30 & 0.14 & 0.02 & 0.05 & 20 & 7 & 0.18 & 0.18 & 0.66 & 0.05 & $\ldots$ & 0.38 & 0.14 \\
\hline G 64-12 & 63904.38 & -3.20 & 0.10 & 0.49 & $\ldots$ & $\ldots$ & $\ldots$ & 1.42 & $\ldots$ & 0.88 & $\ldots$ & $\ldots$ & 0.41 & 0.05 \\
\hline
\end{tabular}


T. Masseron et al.: A holistic approach to carbon-enhanced metal-poor stars

Table 4. Same as Table 2 for stars with $[\mathrm{C} / \mathrm{Fe}]<0.9$.

\begin{tabular}{|c|c|c|c|c|c|c|c|c|c|c|c|c|}
\hline Object & $\begin{array}{c}{[\mathrm{Ba} / \mathrm{Fe}]} \\
(16)\end{array}$ & $\begin{array}{c}\sigma \\
(17) \\
\end{array}$ & $\begin{array}{c}\overline{\mathrm{La} / \mathrm{Fe}]} \\
(18) \\
\end{array}$ & $\begin{array}{c}\sigma \\
(19)\end{array}$ & $\begin{array}{c}\mathrm{Ce} / \mathrm{Fe}] \\
(20) \\
\end{array}$ & $\begin{array}{c}\sigma \\
(21) \\
\end{array}$ & $\begin{array}{c}{[\mathrm{Eu} / \mathrm{Fe}]} \\
(22) \\
\end{array}$ & $\begin{array}{c}\sigma \\
(23)\end{array}$ & $\begin{array}{c}\mathrm{Pb} / \mathrm{Fe}] \\
(24) \\
\end{array}$ & $\begin{array}{c}\sigma \\
(25) \\
\end{array}$ & $\begin{array}{c}\text { Solar ref } \\
(26)\end{array}$ & $\begin{array}{l}\text { References } \\
(27)\end{array}$ \\
\hline \multicolumn{13}{|c|}{ Ba stars } \\
\hline $\mathrm{BD}+18: 5215$ & 1.46 & 0.05 & 1.15 & 0.06 & 1.23 & 0.05 & 0.25 & 0.10 & 0.45 & 0.19 & Gr98 & (46) \\
\hline HR 107 & 0.95 & 0.05 & 0.63 & 0.06 & 0.53 & 0.05 & 0.05 & 0.10 & 0.90 & 0.19 & Gr98 & (46) \\
\hline HD 749 & 1.18 & 0.19 & 1.22 & 0.19 & 1.62 & 0.19 & 0.34 & 0.21 & 0.38 & 0.29 & Gr98 & (46) \\
\hline \multirow[t]{2}{*}{ HD 5424} & 1.04 & 0.20 & 1.28 & 0.05 & 1.66 & 0.16 & 0.49 & 0.05 & 0.91 & 0.10 & As05 & (52) \\
\hline & 1.48 & 0.19 & 1.51 & 0.19 & 1.98 & 0.19 & 0.47 & 0.21 & 1.10 & 0.29 & Gr98 & (46) \\
\hline HD 8270 & 1.11 & 0.05 & 0.96 & 0.06 & 0.95 & 0.05 & 0.33 & 0.10 & 0.50 & 0.19 & Gr98 & (46) \\
\hline HD 12392 & 1.51 & 0.19 & 1.57 & 0.19 & 1.79 & 0.19 & 0.49 & 0.21 & 1.15 & 0.29 & Gr98 & (46) \\
\hline HD 13551 & 1.16 & 0.05 & 0.95 & 0.06 & 1.03 & 0.05 & 0.22 & 0.10 & 0.50 & 0.19 & Gr98 & (46) \\
\hline HD 22589 & 0.88 & 0.05 & 0.66 & 0.06 & 0.57 & 0.05 & 0.22 & 0.10 & -0.15 & 0.19 & Gr98 & (46) \\
\hline HD 24035 & 1.07 & 0.20 & 1.01 & 0.05 & 1.63 & 0.13 & 0.32 & 0.05 & 0.94 & 0.10 & As05 & $(52)$ \\
\hline HD 27271 & 0.88 & 0.19 & 0.68 & 0.19 & 0.78 & 0.19 & 0.32 & 0.21 & 0.31 & 0.29 & Gr98 & (46) \\
\hline HD 48565 & 1.29 & 0.05 & 1.35 & 0.06 & 1.72 & 0.05 & 0.36 & 0.10 & 1.35 & 0.19 & Gr98 & (46) \\
\hline HD 76225 & 1.35 & 0.05 & 1.18 & 0.06 & 1.18 & 0.05 & 0.26 & 0.10 & 0.85 & 0.19 & Gr98 & (46) \\
\hline HD 87080 & 1.48 & 0.05 & 1.70 & 0.06 & 1.85 & 0.05 & 0.67 & 0.10 & 1.05 & 0.19 & Gr98 & (46) \\
\hline HD 89948 & 0.99 & 0.05 & 0.89 & 0.06 & 0.83 & 0.05 & 0.17 & 0.10 & 0.35 & 0.19 & Gr98 & (46) \\
\hline HD 92545 & 1.04 & 0.05 & 0.68 & 0.06 & 0.72 & 0.05 & 0.33 & 0.10 & 0.70 & 0.19 & Gr98 & (46) \\
\hline HD 106191 & 0.88 & 0.05 & 0.62 & 0.06 & 0.73 & 0.05 & 0.21 & 0.10 & 0.65 & 0.19 & Gr98 & (46) \\
\hline HD 107574 & 1.71 & 0.05 & 1.12 & 0.06 & 1.14 & 0.05 & 0.48 & 0.10 & 1.05 & 0.19 & Gr98 & (46) \\
\hline HD 116869 & 1.02 & 0.19 & 0.92 & 0.19 & 1.02 & 0.19 & 0.17 & 0.21 & 0.85 & 0.29 & Gr98 & (46) \\
\hline HD 123396 & 1.30 & 0.19 & 1.23 & 0.19 & 1.74 & 0.19 & 0.51 & 0.21 & 1.20 & 0.29 & Gr98 & (46) \\
\hline HD 123585 & 1.79 & 0.05 & 1.61 & 0.06 & 1.81 & 0.05 & 0.84 & 0.10 & 1.55 & 0.19 & Gr98 & (46) \\
\hline HD 147609 & 1.57 & 0.05 & 1.59 & 0.06 & 1.76 & 0.05 & 0.75 & 0.10 & 0.78 & 0.19 & Gr98 & (46) \\
\hline HD 150862 & 1.03 & 0.05 & 0.76 & 0.06 & 0.67 & 0.05 & 0.21 & 0.10 & 0.70 & 0.19 & Gr98 & (46) \\
\hline HD 168214 & 0.71 & 0.20 & 0.90 & 0.05 & 1.12 & 0.10 & 0.26 & 0.10 & 0.38 & 0.10 & As05 & (52) \\
\hline $\mathrm{HD}$ & 1.20 & 0.05 & 1.12 & 0.06 & 1.35 & 0.05 & 0.30 & 0.10 & 0.95 & 0.19 & Gr98 & (46) \\
\hline HD 2 & 1.23 & 0.10 & 1.37 & 0.05 & 1.41 & 0.15 & 0.58 & 0.10 & 1.30 & 0.10 & As05 & (52) \\
\hline $\mathrm{HD}$ & 0.73 & 0.19 & 0.64 & 0.19 & 0.86 & 0.19 & 0.09 & 0.21 & 0.45 & 0.29 & Gr98 & (46) \\
\hline $\mathrm{HL}$ & 0.99 & 0 & 0.70 & 0.19 & 0.77 & 0.19 & 0.55 & 0.21 & -0.04 & 0.29 & Gr98 & (46) \\
\hline $\mathrm{HL}$ & 0.35 & 0.20 & 0.29 & 0.05 & 0.73 & 0.18 & 0.15 & 0.10 & 0.12 & 0.10 & As05 & (52) \\
\hline 9116 & 0.77 & 0.20 & 0.56 & 0.05 & 0.80 & 0.14 & 0.17 & 0.10 & 0.34 & 0.10 & As05 & $(52)$ \\
\hline HD 222349 & 1.38 & 0.05 & 1.31 & 0.06 & 1.52 & 0.05 & 0.25 & 0.10 & 1.45 & 0.19 & Gr98 & (46) \\
\hline HD 223938 & 1.22 & 0.19 & 1.00 & 0.19 & 1.16 & 0.19 & 0.38 & 0.21 & 0.97 & 0.29 & Gr98 & (46) \\
\hline \multicolumn{13}{|c|}{ rII stars } \\
\hline $\mathrm{CS} 2$ & 0.38 & 0 & $\cdots$ & $\cdots$ & $\ldots$ & $\ldots$ & 1.16 & 0.08 & $\ldots$ & $\ldots$ & Gr96 & (45) \\
\hline CS 29491-069 & 0.34 & 0.19 & & 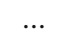 & $\ldots$ & $\ldots$ & 1.06 & 0.15 & 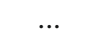 & $\cdots$ & Gr98 & (5) \\
\hline CS 29497-004 & 1.21 & 0.15 & 1.21 & 0.15 & & $\ldots$ & 1.62 & 0.15 & $\ldots$ & $\ldots$ & Gr98 & $(5,47)$ \\
\hline CS 31082-001 & 1.17 & 0.17 & 1.13 & 0.04 & 1.01 & $\ldots$ & 1.63 & 0.05 & 0.40 & $\cdots$ & Gr98 & $(48,49)$ \\
\hline HE 0430-4901 & 0.50 & 0.20 & $\ldots$ & $\ldots$ & $\ldots$ & $\ldots$ & 1.16 & 0.17 & $\ldots$ & $\ldots$ & Gr98 & (5) \\
\hline HE 0432-0923 & 0.72 & 0.18 & $\ldots$ & $\ldots$ & $\ldots$ & $\ldots$ & 1.25 & 0.15 & $\ldots$ & $\ldots$ & Gr98 & (5) \\
\hline HE $1127-1143$ & 0.63 & 0.19 & $\ldots$ & $\ldots$ & $\cdots$ & $\ldots$ & 1.08 & 0.15 & $\ldots$ & $\ldots$ & Gr98 & (5) \\
\hline HE 1219-0312 & 0.51 & 0.25 & 0.91 & 0.17 & $\cdots$ & $\ldots$ & 1.41 & 0.17 & $\ldots$ & $\ldots$ & Gr98 & (5) \\
\hline HE $2224+0143$ & 0.59 & 0.18 & 0.65 & 0.15 & $\ldots$ & $\ldots$ & 1.05 & 0.15 & $\ldots$ & $\ldots$ & Gr98 & (5) \\
\hline HE 2327-5642 & 0.66 & 0.19 & 0.67 & 0.17 & $\ldots$ & $\ldots$ & 1.22 & 0.17 & $\ldots$ & $\ldots$ & Gr98 & $(5)$ \\
\hline \multicolumn{13}{|c|}{ rI stars } \\
\hline Object & $\begin{array}{c}{[\mathrm{Ba} / \mathrm{Fe}]} \\
(16)\end{array}$ & $\begin{array}{c}\sigma \\
(17)\end{array}$ & $\begin{array}{c}\mathrm{La} / \mathrm{Fe}] \\
(18)\end{array}$ & $\begin{array}{c}\sigma \\
(19)\end{array}$ & $\begin{array}{c}\overline{[\mathrm{Ce} / \mathrm{Fe}]} \\
(20)\end{array}$ & $\begin{array}{c}\sigma \\
(21)\end{array}$ & $\begin{array}{c}\overline{[\mathrm{Eu} / \mathrm{Fe}]} \\
(22)\end{array}$ & $\begin{array}{c}\sigma \\
(23)\end{array}$ & $\begin{array}{c}\mathrm{Pb} / \mathrm{Fe}] \\
(24)\end{array}$ & $\begin{array}{c}\sigma \\
(25)\end{array}$ & $\begin{array}{c}\text { Solar ref } \\
\text { (26) }\end{array}$ & $\begin{array}{l}\text { References } \\
\text { (27) }\end{array}$ \\
\hline HD 122563 & -1.02 & 0.12 & -1.02 & 0.10 & -0.64 & 0.18 & -0.52 & 0.17 & $\ldots$ & $\cdots$ & Gr98 & $(25,26,27,29)$ \\
\hline HD 2796 & -0.14 & 0.07 & -0.10 & 0.06 & 0.06 & 0.12 & 0.11 & 0.06 & $\ldots$ & $\ldots$ & Gr98 & $(25,26,27,29)$ \\
\hline HD 186478 & -0.04 & 0.07 & 0.05 & 0.06 & 0.01 & 0.12 & 0.48 & 0.06 & $\cdots$ & $\cdots$ & Gr98 & $(25,26,27,29)$ \\
\hline $\mathrm{BD}+17: 3248$ & 0.69 & 0.07 & 0.66 & 0.06 & 0.49 & 0.12 & 0.93 & 0.06 & $\ldots$ & $\ldots$ & Gr98 & $(25,26,27,29)$ \\
\hline BD -18:5550 & -0.74 & 0.07 & $<-0.91$ & 0.06 & -0.42 & 0.12 & -0.20 & 0.06 & $\ldots$ & $\ldots$ & Gr98 & $(25,26,27,29)$ \\
\hline CD -38:245 & -0.76 & 0.07 & $<0.02$ & 0.06 & 0.71 & 0.12 & $<0.38$ & 0.06 & $\ldots$ & $\ldots$ & Gr98 & $(25,26,27,29)$ \\
\hline BS 16467-062 & $<-1.16$ & 0.07 & $<0.30$ & 0.06 & 1.19 & 0.12 & $<0.76$ & 0.06 & $\ldots$ & $\ldots$ & Gr98 & $(25,26,27,29)$ \\
\hline BS 16477-003 & -0.45 & 0.07 & $<-0.01$ & 0.06 & 0.38 & 0.12 & $<0.25$ & 0.06 & $\ldots$ & $\ldots$ & Gr98 & $(25,26,27,29)$ \\
\hline BS 17569-049 & 0.20 & 0.07 & 0.38 & 0.06 & 0.23 & 0.12 & 0.72 & 0.06 & $\ldots$ & $\ldots$ & Gr98 & $(25,26,27,29)$ \\
\hline CS 22169-035 & -1.19 & 0.07 & $<-0.93$ & 0.06 & -0.34 & 0.12 & $<-0.67$ & 0.06 & $\ldots$ & $\ldots$ & Gr98 & $(25,26,27,29)$ \\
\hline CS 22172-002 & -1.17 & 0.07 & $<-0.01$ & 0.06 & 0.58 & 0.12 & $<0.05$ & 0.06 & $\ldots$ & $\ldots$ & Gr98 & $(25,26,27,29)$ \\
\hline CS 22186-025 & 0.02 & 0.07 & 0.19 & 0.06 & 0.27 & 0.12 & 0.54 & 0.06 & $\ldots$ & $\ldots$ & Gr98 & $(25,26,27,29)$ \\
\hline CS 22189-009 & -1.29 & 0.07 & $<-0.18$ & 0.06 & 0.41 & 0.12 & $<-0.02$ & 0.06 & $\ldots$ & $\ldots$ & Gr98 & $(25,26,27,29)$ \\
\hline CS 22873-055 & -0.45 & 0.07 & -0.47 & 0.06 & -0.09 & 0.12 & -0.17 & 0.06 & & $\cdots$ & Gr98 & $(25,26,27,29)$ \\
\hline CS 22873-166 & -0.70 & 0.07 & -0.77 & 0.06 & -0.34 & 0.12 & -0.30 & 0.06 & $\ldots$ & $\ldots$ & Gr98 & $(25,26,27,29)$ \\
\hline
\end{tabular}


Table 4. continued.

\begin{tabular}{|c|c|c|c|c|c|c|c|c|c|c|c|c|}
\hline Object & $\begin{array}{c}{[\mathrm{Ba} / \mathrm{Fe}]} \\
(16)\end{array}$ & $\begin{array}{c}\sigma \\
(17)\end{array}$ & $\begin{array}{c}{[\mathrm{La} / \mathrm{Fe}]} \\
(18)\end{array}$ & $\begin{array}{c}\sigma \\
(19)\end{array}$ & $\begin{array}{c}{[\mathrm{Ce} / \mathrm{Fe}]} \\
(20)\end{array}$ & $\begin{array}{c}\sigma \\
(21)\end{array}$ & $\begin{array}{c}{[\mathrm{Eu} / \mathrm{Fe}]} \\
(22)\end{array}$ & $\begin{array}{c}\sigma \\
(23)\end{array}$ & $\begin{array}{c}{[\mathrm{Pb} / \mathrm{Fe}]} \\
(24)\end{array}$ & $\begin{array}{c}\sigma \\
(25)\end{array}$ & $\begin{array}{c}\text { Solar ref } \\
\text { (26) }\end{array}$ & $\begin{array}{l}\text { References } \\
(27)\end{array}$ \\
\hline 52 & -0.58 & 0.07 & -0.42 & 0.06 & 0.17 & 0.12 & -0.06 & 0.06 & 5 & - & Gr98 & $(25,26,27,29)$ \\
\hline 96 & 10 & 0.07 & -0.09 & 0.06 & 0.90 & 2 & 47 & 0.06 & & & G & a) \\
\hline & -0.55 & 07 & 0.28 & 0.06 & 19 & 2 & 09 & 06 & & & 8 & 9) \\
\hline & & 7 & 2 & 0.06 & 0 . & 0.12 & 0.86 & .06 & & & & 9) \\
\hline 8 & 1.00 & 0.07 & -0.46 & 0.06 & 0 . & 2 & $<-0.20$ & 0.06 & $\ldots$ & & $\mathrm{Gr}$ & 9) \\
\hline 6 & -0.94 & 0.07 & -0.73 & 0.06 & -0.04 & 0.12 & $<-0.57$ & 0.06 & & & G & 29) \\
\hline 15 & -1.33 & 0.07 & $<-0.54$ & 0.06 & 0.05 & 0.12 & $<-0.28$ & 0.06 & $\cdots$ & ... & $\mathrm{G}_{1}$ & 25,26 \\
\hline & 0.31 & 0.10 & 0.55 & 0.10 & 0.40 & 0.20 & 0.86 & 0.05 & $<1.18$ & 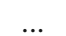 & 55 & (52) \\
\hline & 0.49 & 0. & 0.6 & 0. & 0. & 0 & 1. & 0.06 & $\cdots$ & & $\mathrm{GrC}$ & 29) \\
\hline $\mathrm{CS} 2$ & -0.78 & 0.07 & $<-0.24$ & 0.06 & 0.25 & 0.12 & $<0.02$ & 0.06 & $\cdots$ & $\cdots$ & & 29) \\
\hline 57 & -0.24 & 0.07 & 0.25 & 0.06 & 0.34 & 0.12 & 0.41 & 0.06 & & $\cdots$ & Gr98 & $(25,26$, \\
\hline 014 & -1.77 & 0.07 & -0.11 & 0.06 & 0.48 & 0.12 & $<0.05$ & 0.06 & $\ldots$ & & Gr98 & $(25,26,27,29)$ \\
\hline & -0.89 & 0.07 & $<-0.93$ & 0.06 & -0.34 & 0.12 & -0.42 & 0.06 & 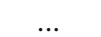 & $\cdots$ & Gr98 & $(25,26,27,29)$ \\
\hline & -0.65 & 0.07 & -0.45 & 0.06 & -0.16 & 0.12 & -0.09 & 0.06 & $\cdots$ & $\ldots$ & Gr98 & $(25,26,27,29)$ \\
\hline-042 & -1.69 & 0.07 & $<-0.58$ & 0.06 & 0.51 & 0.12 & $<-0.22$ & 0.06 & $\ldots$ & $\ldots$ & Gr98 & $(25,26,27,29)$ \\
\hline & -0.90 & 0.07 & -0.61 & 0.06 & -0.32 & 0.12 & -0.25 & 0.06 & $\ldots$ & & & \\
\hline & -0.45 & 0.07 & -0.49 & 0.0 & 0.20 & 0.12 & $<-0.13$ & 0.06 & 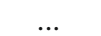 & & & $(25,26$ \\
\hline & -1.88 & 0.07 & $<-0.27$ & 0.06 & 0.42 & 0.12 & $<-0.11$ & 0.06 & $\cdots$ & $\cdots$ & Gr98 & $(25,20$ \\
\hline G 64-12 & -0.25 & $\cdots$ & $\cdots$ & $\cdots$ & $\cdots$ & $\ldots$ & & $\cdots$ & $\ldots$ & $\ldots$ & As05 & (15) \\
\hline
\end{tabular}

